\title{
1 Inference of the SARS-CoV-2 generation time using UK household data
}

2 W.S. Hart ${ }^{1} *$, S. Abbott ${ }^{2}$, A. Endo ${ }^{2}$, J. Hellewell ${ }^{2}$, E. Miller ${ }^{3,4}$, N. Andrews ${ }^{5}$, P.K. Maini ${ }^{1}$, S.

3 Funk $^{2, \dagger}$, R.N. Thompson ${ }^{6,7, \dagger}$

4 Affiliations:

$5 \quad{ }^{1}$ Mathematical Institute, University of Oxford, Oxford, OX2 6GG, UK.

$6{ }^{2}$ Centre for Mathematical Modelling of Infectious Diseases, London School of Hygiene and 7 Tropical Medicine, London, WC1E 7HT, UK.

$8{ }^{3}$ Department of Infectious Disease Epidemiology, Faculty of Epidemiology \& Population 9 Health, London School of Hygiene and Tropical Medicine, London, WC1E 7HT, UK.

$10{ }^{4}$ Immunisation and Countermeasures Division, UK Health Security Agency, London, NW9 $115 \mathrm{EQ}, \mathrm{UK}$.

$12{ }^{5}$ Data and Analytical Sciences, UK Health Security Agency, London, NW9 5EQ, UK.

$13{ }^{6}$ Mathematics Institute, University of Warwick, Coventry, CV4 7AL, UK.

$14{ }^{7}$ Zeeman Institute for Systems Biology and Infectious Disease Epidemiology Research, 15 University of Warwick, Coventry, CV4 7AL, UK.

$16 *$ Correspondence to: william.hart@ keble.ox.ac.uk.

17 These authors contributed jointly to this research

19 Abstract:

The distribution of the generation time (the interval between individuals becoming infected

21 and transmitting the virus) characterises changes in the transmission risk during SARS-CoV-

222 infections. Inferring the generation time distribution is essential to plan and assess public 
23 health measures. We previously developed a mechanistic approach for estimating the

24 generation time, which provided an improved fit to data from the early months of the

25 COVID-19 pandemic (December 2019-March 2020) compared to existing models (Hart et

26 al., 2021). However, few estimates of the generation time exist based on data from later in the

27 pandemic. Here, using data from a household study conducted from March-November 2020

28 in the UK, we provide updated estimates of the generation time. We considered both a

29 commonly used approach in which the transmission risk is assumed to be independent of

30 when symptoms develop, and our mechanistic model in which transmission and symptoms

31 are linked explicitly. Assuming independent transmission and symptoms, we estimated a

32 mean generation time (4.2 days, $95 \%$ credible interval 3.3-5.3 days) similar to previous

33 estimates from other countries, but with a higher standard deviation (4.9 days, 3.0-8.3 days).

34 Using our mechanistic approach, we estimated a longer mean generation time (5.9 days, 5.2-

357.0 days) and a similar standard deviation (4.8 days, 4.0-6.3 days). As well as estimating the

36 generation time using data from the entire study period, we also considered whether the

37 generation time varied temporally. Both models suggest a shorter mean generation time in

38 September-November 2020 compared to earlier months. Since the SARS-CoV-2 generation

39 time appears to be changing, further data collection and analysis is necessary to continue to

40 monitor ongoing transmission and inform future public health policy decisions. 
42 The generation time (or generation interval) of a SARS-CoV-2 infector-infectee pair is

43 defined as the period of time between the infector and infectee each becoming infected

44 (Anderson and May, 1992; Diekmann and Heesterbeek, 2000; Griffin et al., 2020; Svensson,

45 2007; Wallinga and Lipsitch, 2007). The generation time distribution of many infector-

46 infectee pairs characterises the temporal profile of the transmission risk of an infected host

47 (averaged over all hosts and normalised so that it represents a valid probability distribution)

48 (Fraser, 2007). Inferring the generation time distribution of SARS-CoV-2 is important in

49 order to predict the effects of non-pharmaceutical interventions such as contact tracing and

50 quarantine (Ashcroft et al., 2021; Ferretti et al., 2020b; Hart et al., 2021). In addition, the

51 generation time distribution is widely used in epidemiological models for estimating the time-

52

53

54

55

56

57

58 dependent reproduction number from case notification data (Abbott et al., 2020; Fraser, 2007; Gostic et al., 2020; Thompson et al., 2020) and is crucial for understanding the relationship between the reproduction number and the epidemic growth rate (Fraser, 2007; Parag et al., 2021; Park et al., 2020a; Wallinga and Lipsitch, 2007).

The SARS-CoV-2 generation time distribution has previously been estimated using data from known infector-infectee transmission pairs (Ferretti et al., 2020b, 2020a; Hart et al., 2021) or entire clusters of cases (Ganyani et al., 2020; Hu et al., 2021; Sun et al., 2021). These studies involved data (Cheng et al., 2020; Ferretti et al., 2020b; Ganyani et al., 2020; He et al., 2020; Xia et al., 2020; Zhang et al., 2020) collected between December 2019 and April 2020, almost entirely from countries in East and Southeast Asia (with the exception of four transmission pairs from Germany and four from Italy in (Ferretti et al., 2020b)). Evidence from January and February 2020 in China suggested a temporal reduction in the mean generation time due to non-pharmaceutical interventions (Sun et al., 2021). Specifically, 
effective isolation of infected individuals is likely to have reduced the proportion of transmissions occurring when potential infectors were in the later stages of infection, thereby shortening the generation time (Sun et al., 2021). Similarly, two other studies found a decrease in the serial interval (the difference between symptom onset times of an infector and infectee) (Ali et al., 2020) and an increase in the proportion of presymptomatic transmissions (Bushman et al., 2021) in China over the same time period, which can be attributed to symptomatic hosts being isolated increasingly quickly over time.

Despite estimation of the SARS-CoV-2 generation time in Asia early in the pandemic, relatively little is known about the generation time distribution outside Asia, and whether or not any changes have occurred in the generation time since the early months of the pandemic.

77 At the time of writing, we are aware of only one previous study in which the generation time was estimated using data from the UK (Challen et al., 2021). In that study (Challen et al., 2021), data describing symptom onset dates for 50 infector-infectee pairs, collected by Public Health England (PHE; now the UK Health Security Agency) between January and March 2020 as part of the "First Few Hundred" case protocol (Boddington et al., 2021; Public Health England, 2020), were used to infer the generation time distribution. However, since these transmission pairs mostly consisted of international travellers and their household contacts, the authors concluded that their estimates of the generation time may have been biased downwards due to enhanced surveillance and isolation of these cases (Challen et al., 2021).

Here, we use data from a household study (Miller et al., 2021), conducted between March and November 2020, to estimate the SARS-CoV-2 generation time distribution in the UK under two different underlying transmission models. In the first model (the "independent 
transmission and symptoms model"), a parsimonious assumption is made that the generation

92

93 time and the incubation period of the infector are independent (i.e., there is no link between the times at which infectors transmit the virus and the times at which they develop symptoms), as has often been employed in studies in which the SARS-CoV-2 generation time has been estimated (Challen et al., 2021; Ferretti et al., 2020b, 2020a; Ganyani et al., 2020; Hart et al., 2021; Lehtinen et al., 2021) (Table 1). In the second model (the "mechanistic model"), we use a mechanistic approach in which potential infectors progress through different stages of infection, first becoming infectious before developing symptoms (Hart et al., 2021). Infectiousness is therefore explicitly linked to symptoms in the mechanistic model. A feature of the mechanistic model is that individuals with longer incubation periods will (on average) be infectious for longer before developing symptoms, and so generate more transmissions, compared to those with shorter incubation periods. By fitting separately to data from three different time intervals within the study period, we explore whether or not there was a detectable temporal change in the generation time distribution.

\begin{tabular}{|c|c|c|c|c|}
\hline Study & Location & Time period & $\begin{array}{c}\text { Standard } \\
\text { Mean } \\
\text { generation } \\
\text { time (days) } \\
\text { generation } \\
\text { time }\end{array}$ \\
$\begin{array}{c}\text { Ferretti et al. } \\
\text { distribution } \\
\text { (days) }\end{array}$ \\
$\begin{array}{c}\text { (Ferretti et } \\
\text { al., 2020b) }\end{array}$ & Various & $\begin{array}{c}\text { December } \\
2019-\end{array}$ & 5.0 & 1.9 \\
\hline $\begin{array}{c}\text { Ganyani et } \\
\text { al. (Ganyani } \\
\text { et al., 2020) }\end{array}$ & Singapore & $\begin{array}{c}\text { Fanuary- } \\
\text { February } \\
2020\end{array}$ & $\begin{array}{c}5.20(3.78- \\
6.78)\end{array}$ & $\begin{array}{c}1.72(0.91- \\
3.93)\end{array}$ \\
\hline $\begin{array}{c}\text { Ganyani et } \\
\text { al. (Ganyani } \\
\text { et al., 2020) }\end{array}$ & China & $\begin{array}{c}\text { January- } \\
\text { February } \\
2020\end{array}$ & $\begin{array}{c}3.95(3.01- \\
4.91)\end{array}$ & $\begin{array}{c}1.51(0.74- \\
2.97)\end{array}$ \\
\hline $\begin{array}{c}\text { Hart et al. } \\
\text { (Hart et al., } \\
\text { 2021) }\end{array}$ & Various & $\begin{array}{c}\text { December } \\
2019-M a r c h \\
2020\end{array}$ & $5.57(5.08-$ & $2.32(1.83-$ \\
$6.09)$ & $2.91)$ \\
\hline Ferretti et al. & Various & December & 5.5 & 1.8 \\
\hline
\end{tabular}




\begin{tabular}{|c|l|c|c|c|}
\hline $\begin{array}{c}\text { (Ferretti et } \\
\text { al., 2020a) }\end{array}$ & & $\begin{array}{c}\text { 2019-March } \\
2020\end{array}$ & & \\
\hline $\begin{array}{c}\text { Challen et al. } \\
\text { (Challen et } \\
\text { al., 2021) }\end{array}$ & UK & $\begin{array}{c}\text { January- } \\
\text { March 2020 }\end{array}$ & $\begin{array}{c}4.8(4.3- \\
5.41)\end{array}$ & $1.7(1.0-2.6)$ \\
\hline
\end{tabular}

Table 1. Previous SARS-CoV-2 generation time estimates. Estimates of the mean and standard deviation of the generation time distribution, obtained under the assumption of independent transmission and symptoms. 95\% credible intervals are shown in brackets where available.

\section{RESULTS}

112 Inferring the generation time from UK household data

113 We fitted two models of infectiousness (the independent transmission and symptoms model and the mechanistic model) to data collected from 172 UK households in a study (Miller et al., 2021) conducted by PHE between March and November 2020 (Figure 1-Source Data 1).

116 Each household was recruited to the study following a confirmed SARS-CoV-2 infection,

117 and all household members were then followed to investigate whether or not they became

118 infected (this was determined through PCR and antibody testing). If a household member was 119 infected and developed symptoms, their symptom onset date was recorded (see Materials and 120 Methods).

122 In our previous work (Hart et al., 2021), we fitted the same two models of infectiousness to 123 data from infector-infectee transmission pairs collected in the early months of the COVID-19 pandemic. Here, we adapted the approach presented in that article (Hart et al., 2021) in order to estimate the generation time using household transmission data. Specifically, we used data augmentation MCMC, augmenting the observed data with estimated times of infection and

127 the precise times at which symptomatic infected hosts developed symptoms. This enabled us 128 (in the likelihood function) to account for uncertainty about exactly who-infected-whom 
within a household by summing together likelihood contributions corresponding to infection by different possible infectors. In addition, we corrected for the regularity of household contacts to derive more widely applicable estimates of the generation time. We did this by including a factor in the likelihood to account for each infected individual avoiding infection from household contacts that occurred prior to their actual time of infection (see Materials and Methods for full details of our approach).

For the two fitted models, we calculated posterior estimates of the mean (Figure 1A) and standard deviation (Figure 1B) of the generation time distribution, in addition to the proportion of transmissions occurring prior to symptom onset (among infectors who develop symptoms; Figure 1C) and the overall infectiousness parameter, $\beta_{0}$ (see Materials and Methods; Figure 1D). Under the commonly used independent transmission and symptoms model, we obtained a point estimate of 4.2 days (95\% credible interval (CrI) 3.3-5.3 days) for

142 the mean generation time (Figure 1A, blue violin; as in other studies using data augmentation 143 MCMC (Cauchemez et al., 2004; Ferguson et al., 2005), we calculated point estimates for each model using the posterior means of fitted model parameters because the mode of the joint posterior distribution could not easily be calculated from the output of the MCMC procedure). This value is similar to a previous estimate obtained using data from China by

147 (Ganyani et al., 2020). It is slightly lower than estimates for Singapore obtained by (Ganyani 148 et al., 2020) and for several countries (predominantly in Asia) obtained by (Ferretti et al., 149 2020b) (Table 1), although those estimates lie within our credible interval. On the other hand, our estimated standard deviation of 4.9 days (95\% CrI 3.0-8.3 days; Figure 1B, blue violin) is

151 substantially higher than previous estimates (Table 1). Using our mechanistic model, we 152 obtained a higher estimate for the mean generation time of 5.9 days (95\% CrI 5.2-7.0 days; 153 Figure 1A, red violin), and a similar estimate for the standard deviation (4.8 days, 95\% CrI 
A.

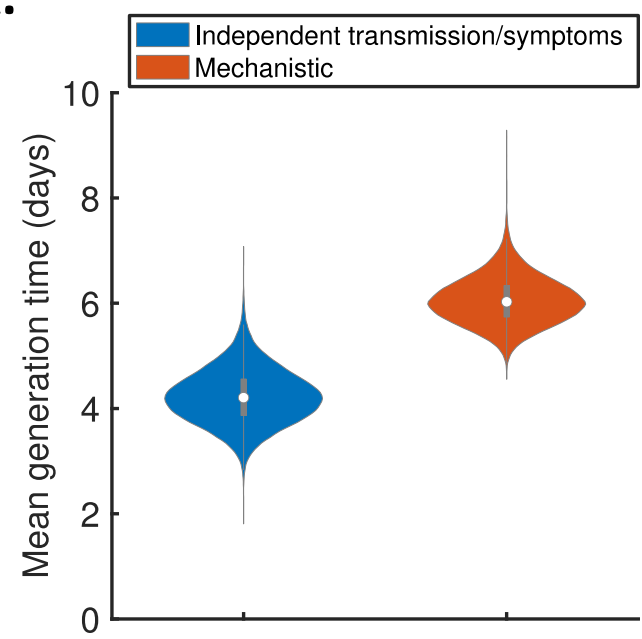

C.

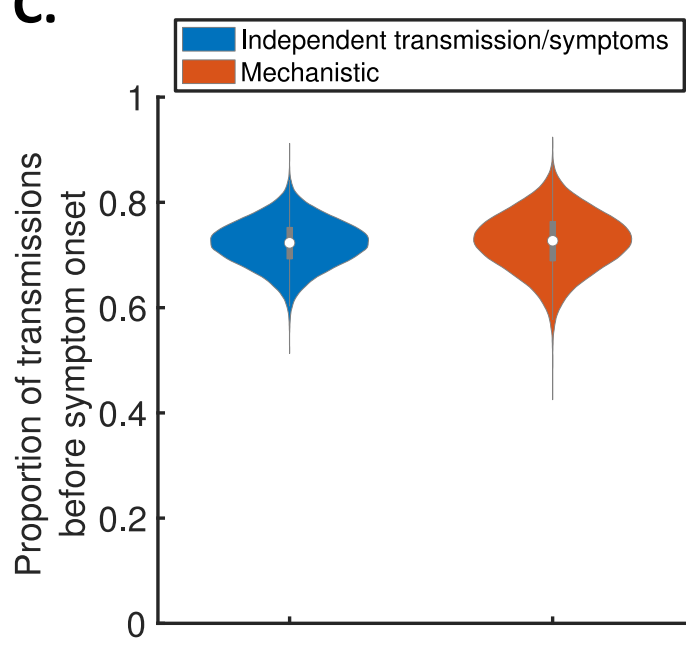

B.

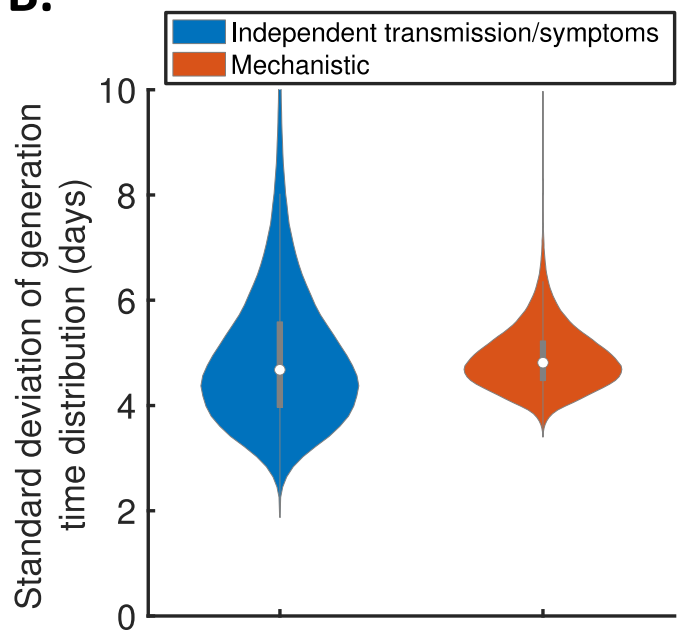

D.

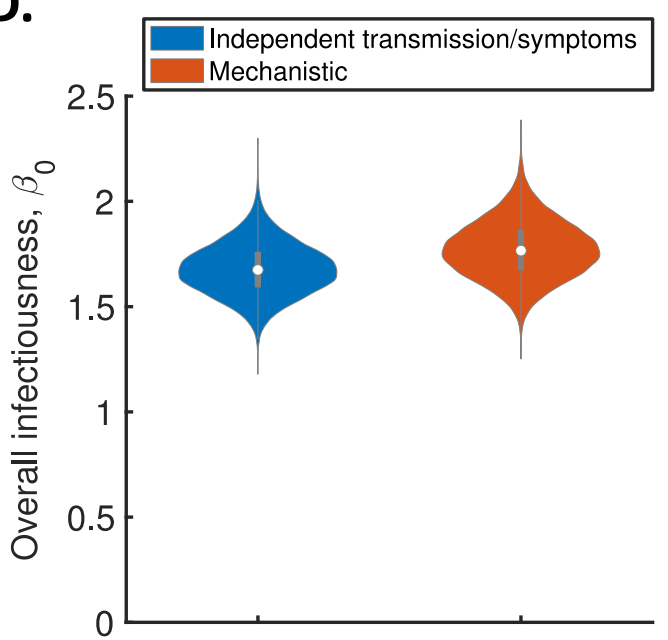

158 Figure 1. Comparison of posterior predictions. Violin plots indicating posterior distributions of the mean (A) and standard deviation (B) of the generation time distribution, proportion of transmissions occurring prior to

160 symptom onset (among infectors who develop symptoms; C), and overall infectiousness parameter, $\beta_{0}$ (describing the expected number of household transmissions generated by a single infected host in a large, otherwise entirely susceptible, household; D). We show results obtained both using a model in which infectiousness is assumed to be independent of when symptoms develop ("independent transmission and 
symptoms model", blue), and using the mechanistic model from (Hart et al., 2021) in which infectiousness is explicitly linked to symptoms ("mechanistic model", red).

The two models gave similar posterior distributions for the proportion of transmissions prior to symptom onset (Figure 1C). Specifically, point estimate values of model parameters led to an estimated proportion of transmissions prior to symptom onset of 0.72 (95\% CrI $0.63-0.80)$ for the independent transmission and symptoms model, and 0.73 (95\% CrI 0.61-0.83) for the mechanistic model. These estimates are higher than obtained in some previous studies in which the infectiousness profile of SARS-CoV-2 infected hosts at each time since infection and/or time since symptom onset has been estimated (Ashcroft et al., 2020; Ferretti et al., 2020b, 2020a; He et al., 2020). On the other hand, our point estimates for the two models both lie within the $95 \%$ credible interval obtained for the mechanistic model in our previous work (Hart et al., 2021) (0.53-0.77, point estimate 0.65). Similar or higher estimates also exist in the wider literature (Casey-Bryars et al., 2021; Ganyani et al., 2020; Tindale et al., 2020).

Posterior distributions for all fitted model parameters are shown in Figure 1-figure supplement 1 and Figure 1-figure supplement 2, and point estimates and 95\% credible intervals are given in Appendix 1-table 2 and Appendix 1-table 3. Since only the likelihood with respect to augmented data was calculated in the MCMC procedure, direct comparisons

184 of the goodness of fit between the models were not readily available. However, comparing 185 model predictions of the distribution of the interval between successive symptom onset dates to the analogous distribution in the household data indicated that both models provided a similar fit to the data (Figure 1-figure supplement 3). 
In Figure 1 (and elsewhere, unless otherwise stated), we characterise the generation time distribution assuming that a constant supply of susceptible individuals are available to infect.

191 This distribution corresponds to the normalised expected infectiousness profile of a host at each time since infection, and is widely applicable to transmission outside of, as well as within, households. However, realised household generation times are expected to be shorter than the estimates shown in Figure 1. This is due to the depletion of susceptible household members before longer generation times can be obtained, especially in small households (Cauchemez et al., 2009; Fraser, 2007; Park et al., 2020b). As a result, we also predicted the mean and standard deviation of realised generation times within the study households (Figure 1-figure supplement 4A-B), accounting for the precise distribution of household sizes in the study. For both the independent transmission and symptoms model and the mechanistic model, the mean (point estimates 3.6 days and 4.9 days for the two models, respectively) and standard deviation (3.8 days and 4.1 days) of realised household generation times were lower than our main generation time estimates shown in Figure 1. Since household transmission typically occurs earlier in the infector's course of infection than indicated by the estimates shown in Figure 1, we predicted a higher proportion of presymptomatic transmissions within the study households (Figure 1-figure supplement 4C) compared to the estimates in Figure $1 \mathrm{C}$.

208 For both models, we then used point estimates of fitted model parameters to infer the 209 distributions of the generation time (Figure 2A), the time from onset of symptoms to 210 transmission (TOST; Figure 2B) and the serial interval (Figure 2C). The TOST distribution

211 (which characterises the relative expected infectiousness of a host who develops symptoms at 212 each time from symptom onset, as opposed to from infection (Ashcroft et al., 2020; Ferretti et 213 al., 2020a; He et al., 2020; Lehtinen et al., 2021; Wells et al., 2021)) obtained using the 
mechanistic model was more concentrated around the time of symptom onset compared to

215 that predicted assuming independent transmission and symptoms (Figure 2B), as we found in

216 our previous work (Hart et al., 2021). In contrast, the estimated serial interval distributions

217 were similar for the two models (Figure 2C). The means and standard deviations of the

218 distributions shown in Figure 2 are given in Appendix 1-table 4.

219

220

221

222

223

224

225

226

227

228

229

230

231

232

233

234

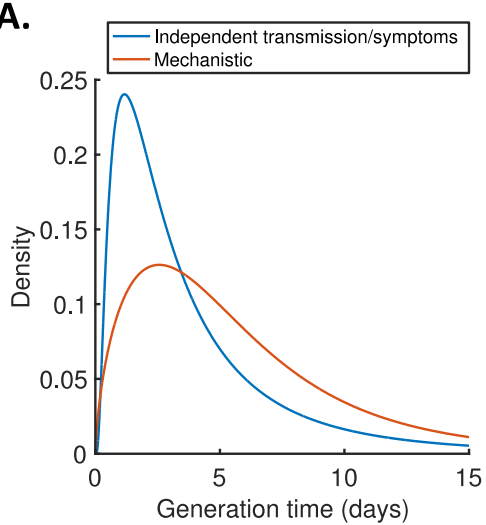

B.

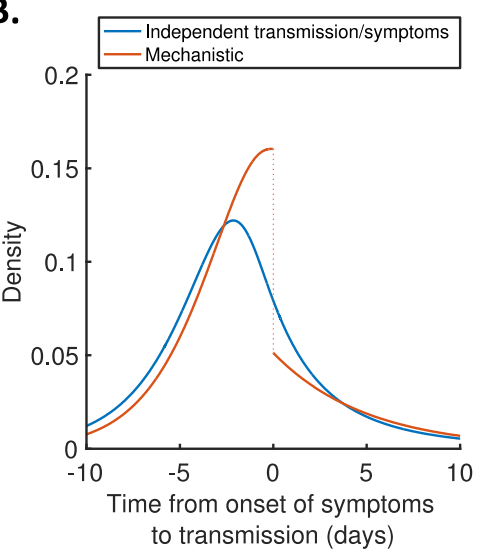

c.

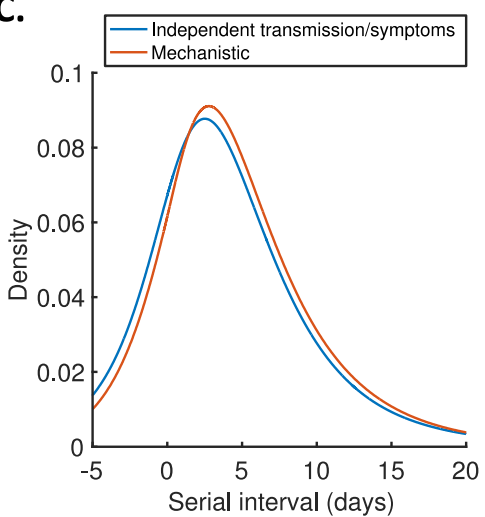

Figure 2. Generation time, TOST and serial interval distributions. Inferred generation time (A), TOST (B) and serial interval (C) distributions for the two models, obtained using point estimate (posterior mean)

parameters. The means and standard deviations of these distributions are given in Appendix 1-table 4. Similarly to (Hart et al., 2021), the discontinuity in the red curve in Figure 2B occurs because different transmission rates were fitted for infectors in the presymptomatic infectious and symptomatic infectious periods. The reduction in transmission following symptom onset can be attributed to changes in behaviour in response to symptoms (Manfredi and D’Onofrio, 2013).

\section{Temporal variation in the generation time distribution}

To explore whether or not the generation time distribution changed during the study period, we separately fitted the independent transmission and symptoms model to the data from households in which the index case was recruited in (i) March-April, (ii) May-August, or (iii) September-November 2020 (Figure 3). We chose these time periods to ensure the numbers of households recruited into the study during each interval were similar (Figure 3-figure supplement 1). 
A.

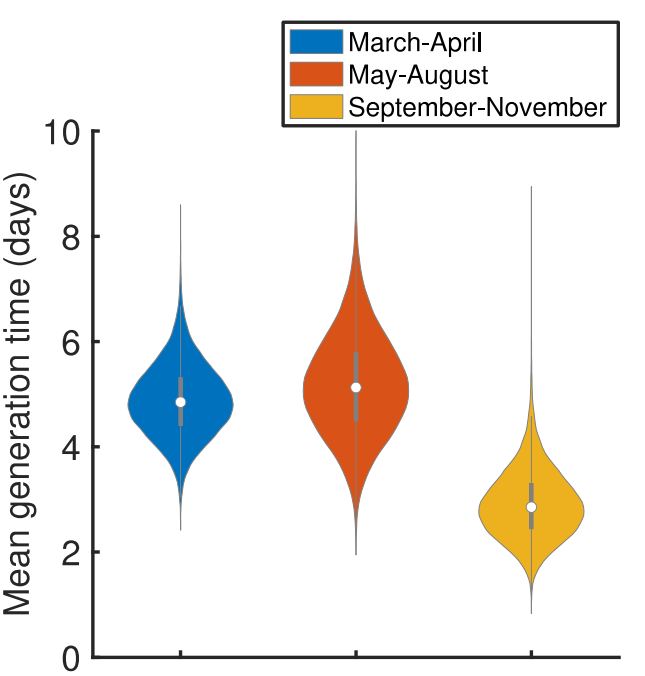

C.

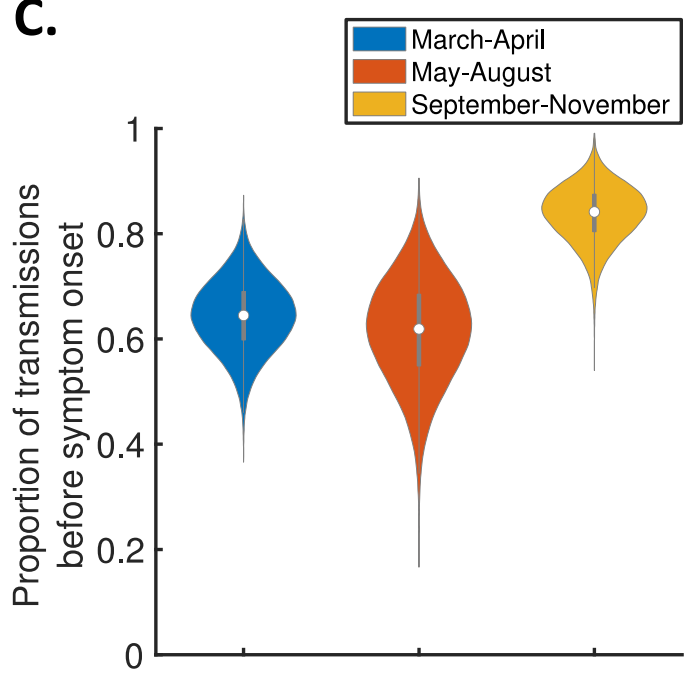

B.
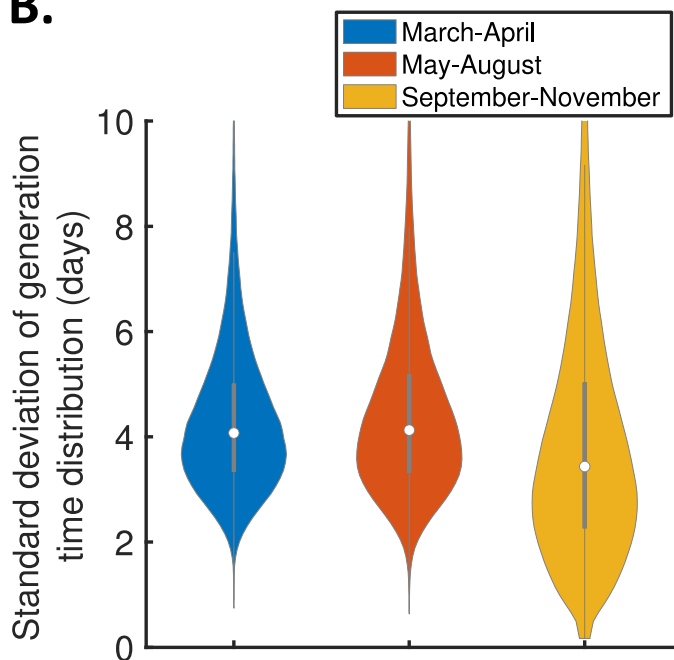

D.
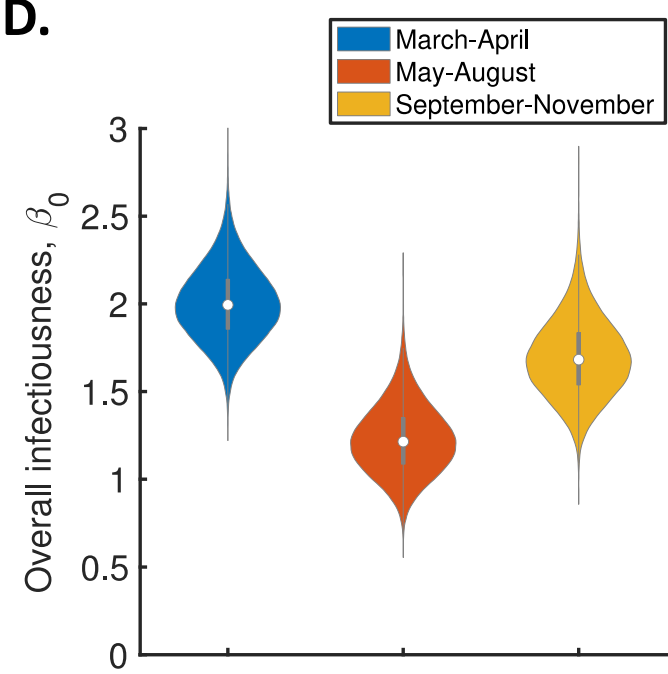

Figure 3. Temporal changes in the generation time. Violin plots indicating posterior distributions of the mean

(A) and standard deviation (B) of the generation time distribution, proportion of transmissions occurring prior to symptom onset (among infectors who develop symptoms; C), and overall infectiousness parameter, $\beta_{0}(\mathrm{D})$, for the independent transmission and symptoms model fitted to data from March-April (blue), May-August (red) or September-November 2020 (orange).

242 The results shown in Figure 3A suggest a shorter mean generation time in September-

243 November 2020 (2.9 days, 95\% CrI 1.8-4.3 days) compared to earlier months (4.9 days, 95\%

244 CrI 3.6-6.3 days, for March-April and 5.2 days, 95\% CrI 3.4-7.2 days, for May-August).

245 Comparing the posterior estimates for May-August and September-November (the red and 
orange violins in Figure 3A, respectively) indicated a 97\% posterior probability of a shorter

247 mean generation time in the later of these two time periods. A similar temporal reduction in

248 the mean generation time was found when we instead fitted the mechanistic model to the data

249 from the three time intervals (Figure 3-figure supplement 2). Estimates of the mean

250 generation time using the mechanistic model were 6.5 days (95\% CrI 5.6-8.1 days) for

251 March-April, 7.1 days (95\% CrI 5.7-9.6 days) for May-August, and 5.1 days (95\% CrI 4.3-

252

6.4 days) for September-November, with a $98 \%$ posterior probability of a shorter mean

253

generation time in September-November than May-August. We also used point estimates of

254

model parameters to compare the distributions of the generation time, TOST and serial

255

interval between the time periods (Figure 3-figure supplement 3), with both models

256

indicating that the transmission risk peaked earlier in infection for individuals infected in

257

September-November compared to earlier months (Figure 3-figure supplement 3, panels A

258 and D).

259

260

Figure $3 \mathrm{C}$ shows posterior estimates for the proportion of transmissions occurring prior to

symptom onset (among symptomatic infectors) across the three time periods for the

262

independent transmission and symptoms model, indicating a very high proportion of

263

presymptomatic transmissions in September-November $(0.83,95 \%$ CrI 0.72-0.93) compared

264

to lower estimates for March-April $(0.64,95 \%$ CrI 0.51-0.77) and May-August $(0.62,95 \%$

265

CrI 0.41-0.79). Our results for the mechanistic model indicate a similar temporal increase in the proportion of presymptomatic transmissions during the study period (Figure 3-figure supplement 2C).

269 To explore the lower estimated generation time for September-November further, we also

270 fitted the independent transmission and symptoms model to the data from each of these 
months individually (Figure 3-figure supplement 4). The shorter estimated generation time compared to earlier in the pandemic was consistent across each of the three months (Figure 3-

273 figure supplement 4A). We note that, while the Alpha (B.1.1.7) variant had begun to emerge

274 in the UK by the end of the study period (Public Health England, 2021), genomic

275 surveillance as part of the study showed that this variant caused infections in only two study

276 households. This variant was therefore unlikely to have been responsible for the temporal 277 reduction in the generation time that we observed.

278

279 In Figure 3-figure supplement 5, we show the posterior distributions of the fitted parameters 280 for the mechanistic model over the different time periods. The fitted parameters represent the mean duration of the presymptomatic infectious period (expressed as a proportion of the mean incubation period; Figure 3-figure supplement 5A), the mean duration of the symptomatic infectious period (Figure 3-figure supplement 5B), and the relative infectiousness of presymptomatic infectious hosts compared to those with symptoms (Figure 3-figure supplement 5C). However, there was substantial overlap in the credible intervals of posterior estimates of each parameter between the three time periods. We were therefore unable to identify the precise parameter(s) responsible for the decrease in generation time and increase in the proportion of presymptomatic transmissions that we observed.

290 Sensitivity analyses

291 When we fitted the two models to the household transmission data, we assumed that each household transmission chain was initiated by a single primary case and all other infected household members were infected from within the household. However, we also extended our framework to account for the possibility of co-primary cases (Appendix, Figure 1-figure supplement 5 and Figure 3-figure supplement 6). This led to slightly higher estimates of the 
mean generation time (Figure 1-figure supplement 5A) under each model compared to the corresponding estimates shown in Figure 1A, with point estimates of 4.8 days (95\% CrI 3.66.3 days) for the independent transmission and symptoms model and 6.8 days (95\% CrI 5.78.6 days) for the mechanistic model. Estimates of the standard deviation of the generation time distribution were similar to those in Figure 1B (Figure 1-figure supplement 5B; point estimates were 4.8 days (95\% CrI 2.9-7.9 days) for the independent transmission and symptoms model and 5.1 days (95\% CrI 4.0-6.9 days) for the mechanistic model). As part of the fitting procedure, we estimated the probability that each household member was infected during the primary transmission event (Figure 1-figure supplement 5E), obtaining point estimates of 0.17 (95\% CrI 0.02-0.33) under the independent transmission and symptoms model and 0.27 (95\% CrI 0.10-0.41) under the mechanistic model. We also repeated the analyses in Figure 3 but accounting for the possibility of co-primary cases (Figure 3-figure supplement 6). Our main qualitative finding remained unchanged: the generation time was found to decrease during the study period (Figure 3-figure supplement 6A).

In the independent transmission and symptoms model, we assumed that both the generation time and incubation period followed lognormal distributions. The mean and standard deviation of the generation time distribution were estimated by fitting the model to the

314 household transmission data. In the fitting procedure, we assumed that the incubation period

315 followed a lognormal distribution that was obtained in a previous meta-analysis (McAloon et 316 al., 2020). In contrast, we assumed in our mechanistic approach that each infection could be 317 decomposed into three gamma distributed stages (latent, presymptomatic infectious and 318 symptomatic infectious), so that the incubation period was also gamma distributed (with the 319 same mean and standard deviation as the lognormal distribution obtained by (McAloon et al., 320 2020)). An expression for the generation time distribution in the mechanistic model, which 
does not take a simple parametric form, is given in the Appendix. However, we conducted

322

323

324

325

326

327

328

329

330

331

332

333

334

335

336

337

338

339

340

341

342

343

344

345

supplementary analyses in which we instead assumed that either the generation time (Figure

1-figure supplement 6) or incubation period (Figure 1-figure supplement 7) in the

independent transmission and symptoms model was gamma distributed. In both cases, we obtained similar results to those shown for that model in Figure 1.

We also relaxed the assumption of a fixed incubation period distribution (Figure 1-figure supplement 8), using the confidence intervals obtained by (McAloon et al., 2020) to account for uncertainty in the incubation period distribution (Figure 1-figure supplement 8A-B). For both the independent transmission and symptoms model and the mechanistic model, accounting for this uncertainty did not substantially affect posterior estimates of either the mean (Figure 1-figure supplement 8C) or the standard deviation (Figure 1-figure supplement 8D) of the generation time distribution.

In our main analyses, we assumed that household transmission was frequency-dependent, so that the force of infection exerted by an infected household member on each susceptible household member scales with $1 / n$, where $n$ is the household size (Cauchemez et al., 2014, 2004). However, since some studies of influenza virus transmission in households have found transmission to lie somewhere in between frequency- and density-dependent (Endo et al., 2019; Ferguson et al., 2005), we also considered alternative possibilities where infectiousness scales with $n^{-\rho}$, for different values of $\rho$. In Figure 1-figure supplement 9A-C, we compared estimates under our baseline value of $\rho=1$ (frequency-dependent transmission) with those obtained assuming either $\rho=0$ (density-dependent transmission) or the intermediate possibility of $\rho=0.5$ considered by (Endo et al., 2019). In addition, we conducted an analysis in which the dependency, $\rho$, was estimated alongside other model parameters 
346 (Figure 1-figure supplement 9D). We found that our estimates of the mean and standard

347 deviation of the generation time distribution were robust to the assumed value of $\rho$ (Figure 1-

348 figure supplement 9A-B). However, when the value $\rho$ was fitted (Figure 1-figure supplement

349 9D), we estimated a value of 1.0 (95\% CrI 0.6-1.5). This supported our assumption of

350 frequency-dependent transmission, although the credible interval was relatively wide. In

351 addition, we considered the possibility that infectiousness instead scales with $1 /(n-1)$, so

352 that the infector under consideration is not included in this scaling, and again obtained similar

353 estimates of the mean and standard deviation of the generation time distribution compared to

354 those shown in Figure 1 (Figure 1-figure supplement 10).

355

356 We also considered the sensitivity of our results to the assumed relative infectiousness of asymptomatic infected hosts (Figure 1-figure supplement 11). In most of our analyses, we assumed that the expected infectiousness of an infected host who remained asymptomatic throughout infection was a factor $\alpha_{A}=0.35$ times that of a host who develops symptoms, at each time since infection (McAloon et al., 2020). However, similar estimates of the mean (Figure 1-figure supplement 11A) and standard deviation (Figure 1-figure supplement 11B) of the generation time distribution were obtained when we instead assumed $\alpha_{A}=0.1$ or $\alpha_{A}=1.27$ (these values corresponded to the lower and upper confidence bounds obtained in (Buitrago-Garcia et al., 2020)). Lower values of $\alpha_{A}$ did lead to slightly higher estimates of the overall infectiousness of infectors who developed symptoms, $\beta_{0}$ (Figure 1-figure supplement 11D). However, this effect was minimal, likely because very few cases in the household study were asymptomatic (27 out of 357$)$.

Finally, we explored the robustness of our results to the exclusion of household members of unknown infection status (see Materials and Methods), considering the extreme possibilities 

where these individuals were instead assumed to have either all remained uninfected, or all become infected (Figure 1-figure supplement 12). Although the estimates of $\beta_{0}$ were affected by this assumption (Figure 1-figure supplement 12D), the estimated generation time distribution was robust to the assumed infection status of these hosts (Figure 1-figure supplement 12A-B).

\section{DISCUSSION}

In this study, we estimated the generation time distribution of SARS-CoV-2 in the UK by fitting two different models to data describing the infection status and symptom onset dates of individuals in 172 households. The first model was predicated on an assumption that transmission and symptoms are independent. While this assumption has often been made in previous studies in which the SARS-CoV-2 generation time has been estimated (Challen et al., 2021; Deng et al., 2020; Ferretti et al., 2020b; Ganyani et al., 2020; Knight and Mishra, 2020), it is not an accurate reflection of the underlying epidemiology (Bacallado et al., 2020; Lehtinen et al., 2021). Therefore, we also considered a mechanistic model based on compartmental modelling, which was shown in our earlier work (Hart et al., 2021) to provide an improved fit to data from 191 SARS-CoV-2 infector-infectee pairs compared to previous models that have been used to estimate the generation time. Here, infection times and the order of transmissions within households were unknown, whereas in (Hart et al., 2021) the direction of transmission was assumed to be known for each infector-infectee pair. For that reason, we needed to extend the statistical inference methods underlying our previous work (Hart et al., 2021) to fit the two models to household data. To do this, we used a data augmentation MCMC approach similar to previous studies of household influenza virus transmission (Cauchemez et al., 2009, 2004; Ferguson et al., 2005). 
Under the model assuming independent transmission and symptoms, we estimated a mean generation time of 4.2 days (95\% CrI 3.3-5.3 days) and a standard deviation of 4.9 days (95\%

398

CrI 3.0-8.3 days). The estimate of the mean generation time was comparable to previous estimates obtained under this assumption using data from elsewhere (Ferretti et al., 2020b, 2020a; Ganyani et al., 2020) (Table 1). On the other hand, while our credible interval for the standard deviation was wide, the estimates obtained in those previous studies (Ferretti et al., 2020b, 2020a; Ganyani et al., 2020) all lay below our lower 95\% credible limit of 3.0 days. One potential cause of this disparity is the difference in isolation policies for symptomatic hosts between countries. In particular, the UK's policy of self-isolation may be expected to lead to a longer-tailed generation time distribution compared to countries with a policy of isolation outside the home, since under home isolation, some within-household transmission is likely to occur even following isolation. Isolation outside the home was commonplace in the East and Southeast Asian countries where the majority of the data underlying the estimates by (Ferretti et al., 2020b, 2020a; Ganyani et al., 2020) were collected.

Using the mechanistic model, we predicted a higher mean generation time of 5.9 days $(95 \%$ CrI 5.2-7.0 days) compared to the value estimated under the assumption of independent transmission and symptoms. On the other hand, the inferred serial intervals for the independent transmission and symptoms model and mechanistic model were more similar (Figure 2C), with means of 4.2 days and 4.7 days, respectively. Temporal information in our household transmission data consisted mostly of symptom onset dates, with very few hosts testing positive before developing symptoms. Therefore, the variation in estimates of the generation time between the models can be attributed to differences in the assumed relationships between the generation time and serial interval under those models. For the independent transmission and symptoms model, the generation time and serial interval 
distributions have the same mean, as is commonly assumed to be the case (Lehtinen et al., 2021). However, this was not true for the mechanistic model, in which hosts with longer presymptomatic infectious periods generate (on average) a higher number of transmissions. As a result, under the mechanistic model, a randomly chosen infection is likely to arise from an infector with a longer than expected incubation period, thereby leading to a longer generation time than serial interval (an analytical expression for the exact difference between the mean generation time and serial interval for that model is derived in the Appendix).

Our results do not indicate any clear difference in goodness of fit to the data between the two models (Figure 1-figure supplement 3). A range of factors should therefore be considered when deciding which of our estimates of epidemiological parameters to use in subsequent analyses. Although any model requires simplifying assumptions to be made, our mechanistic approach allows the standard assumption of independent transmission and symptoms to be relaxed by providing a mechanistic underpinning to the relationship between the times at 435 which individuals display symptoms and become infectious. Furthermore, as described 436 above, this model was shown in our previous work (Hart et al., 2021) to provide a better fit to an earlier SARS-CoV-2 dataset than a model assuming independence between transmission and symptoms (in (Hart et al., 2021), the simpler setting of transmission pairs rather than households facilitated direct model comparison). On the other hand, the independent

440 transmission and symptoms model has the advantage of producing an estimated generation 441 time distribution with a simple parametric form. The choice of estimates to use may also 442 depend on precisely what the estimates are being used for. For example, the generation time 443 distribution inferred under the assumption of independent transmission and symptoms may be 444 better suited for use in some models for estimating the time-dependent reproduction number, 445 since those models often also involve the assumption that transmission and symptoms are 
independent (Abbott et al., 2020). In contrast, the parameter estimates from our mechanistic

447 approach correspond naturally to parameters in compartmental epidemic models.

448

449 By fitting separately to data from three different intervals within the study period (March-

450 November 2020), we investigated whether or not the generation time distribution in the UK changed as the pandemic progressed. Our results indicate a lower mean generation time in September-November compared to earlier months (Figure 3A). One possible explanation for this is a higher proportion of time spent indoors in colder months leading to an increased transmission risk, particularly in the early stages of infection before symptoms develop (since symptomatic infected hosts are still likely to self-isolate). This explanation is consistent with our finding in Figure 3C of a higher proportion of transmissions occurring prior to symptom onset in September-November compared to March-April and May-August.

458

While behavioural changes may have been responsible for our finding of a temporal decrease in the generation time, an alternative explanation could be that evolutionary changes in the SARS-CoV-2 virus that occurred during the study period affected the generation time. For example, the B.1.177 lineage emerged in Spain in early summer 2020, and became the dominant SARS-CoV lineage in the UK around the beginning of October 2020 (Vöhringer et

464 al., 2021). Subsequently, the Alpha (B.1.1.7) variant, which was first detected in September 465 2020, became dominant in the UK in December 2020 (Public Health England, 2021). The 466 Alpha variant has been shown to possess different characteristics than earlier variants (Davies et al., 2021; Volz et al., 2021), causing an increased epidemic growth rate in the UK that has been attributed to an increase in transmissibility of 43-90\% (Davies et al., 2021). While in 469 principle evolutionary changes could explain the variation in the generation time that we 470 observed, sequencing data show that the Alpha variant was responsible for infections in only 
471 two households within our dataset. Consequently, the Alpha variant was not responsible for

472 our main finding of a temporally decreasing generation time, and additional data are required

473 to quantify the impact of the emergence of that variant (and subsequent variants, such as the

474 Delta (B.1.617.2) and Omicron (B.1.1.529) variants) on the SARS-CoV-2 generation time.

475

476 In data collected from infector-infectee transmission pairs, shorter generation times are

477 expected to be over-represented at times when case numbers are rising (Britton and Scalia

478 Tomba, 2019; Ferretti et al., 2020b; Lehtinen et al., 2021) and vice versa. While we used data

479 from households (rather than transmission pairs) in our analyses, a similar effect may have

480 contributed to our shorter estimated mean generation time for September-November 2020

481 (national case numbers were mostly increasing in September-October 2021) compared to

482 earlier months of the study (in which case numbers were mostly decreasing) (Knock et al.,

483 2021; Pouwels et al., 2021). However, we estimated the mean generation time to be similar in

484 November (when case numbers were mostly decreasing (Knock et al., 2021; Pouwels et al.,

485 2021)) compared to September and October (Figure 3-figure supplement 4), suggesting that

486 this effect of background epidemic dynamics alone did not drive the temporal changes in

487 generation time that we observed. We note, however, that sample sizes for individual months

488 were small (Figure 3-figure supplement 1). Extending our household inference framework to

489 explicitly account for background epidemic dynamics in generation time estimates (similar to

490 methods that have been developed for transmission pair data (Britton and Scalia Tomba,

491 2019; Ferretti et al., 2020b)) is an avenue for future work.

492

493 Our finding of a temporal decrease in the mean generation time during the study period

494 highlights the importance of obtaining up-to-date generation time estimates specific to the

495 location under study. Should variations in the generation time distribution occur and not be 
accounted for, estimates of the time-dependent reproduction number may be incorrect (Park

497 et al., 2021; Wallinga and Lipsitch, 2007). Specifically, if the mean generation time is shorter than assumed, then the true value of the time-dependent reproduction number is likely to be closer to one than the inferred value (Wallinga and Lipsitch, 2007) (and vice versa).

500

501 One advantage of our approach compared to previous studies in which the SARS-CoV-2 502 generation time has been estimated (Ferretti et al., 2020b, 2020a; Ganyani et al., 2020; Hart et

503 al., 2021) is that we are able to include the contribution of asymptomatic infected hosts to

504 household transmission chains in our analyses. We showed that our estimated generation time 505 distribution was robust to the assumed relative infectiousness of infected hosts who remained 506 asymptomatic, $\alpha_{A}$ (Figure 1-figure supplement 11). Similarly, while we assumed frequencydependent household transmission in most of our analyses, we found that the exact relationship between the household size and transmission had little effect on our estimates of

509 the mean and standard deviation of the generation time distribution (Figure 1-figure

510 supplement 9 and Figure 1-figure supplement 10). We also considered estimating the exponent governing the dependency of transmission on household size (Figure 1-figure supplement 9D). This supported our assumption of frequency-dependent transmission, and is

513 consistent with the finding of an inverse relationship between household size and secondary

514 attack rate in the household study underlying our analyses (Miller et al., 2021). In previous

515 studies of influenza transmission within households, evidence has been found both in favour

516 of (Cauchemez et al., 2004) and against (Endo et al., 2019) frequency-dependent

517 transmission.

518

519 While our generation time estimates were robust to the assumed relative infectiousness of 520 infected hosts who remained asymptomatic and whether transmission was assumed to be 
521

522

523

524

525

526

527

528

529

530

531

532

533

534

535

536

537

538

539

540

541

542

543 We also note that, while our dataset involved a larger sample size than used in most other

544 studies in which the SARS-CoV-2 generation time was estimated (Ferretti et al., 2020a,

545 2020b; Ganyani et al., 2020; Hart et al., 2021), the demographics of the study households

frequency- or density-dependent, extending our approach to account for the possibility that household transmission chains originated with multiple co-primary cases led to slightly higher estimates of the generation time (Figure 1-figure supplement 5) compared to our main estimates (Figure 1). Despite the overall higher estimated generation time, our main qualitative finding of a temporal decrease in the generation time held when co-primary cases were incorporated (Figure 3-figure supplement 6).

Like any mathematical modelling study, our approach has some limitations. We used household data in our analyses, whereas some characteristics of wider community transmission may differ from those of transmission within households. However, we corrected for the regularity of household contacts to estimate the (expected) infectiousness profile of an infected host at each time since infection (accounting for behavioural factors), which provides a widely applicable generation time estimate (Figure 1). Specifically, the infectiousness profile gives the generation time distribution under the assumption that a constant supply of susceptible individuals are available throughout the host's course of infection. This distribution can then be conditioned to specific population structures, as we demonstrated by estimating the realised generation time distribution within the study households (Figure 1-figure supplement 4). The household generation time estimates shown in Figure 1-figure supplement 4 are shorter than our main generation time estimates (Figure 1), due to the regularity of household contacts and the depletion of susceptible individuals within households before longer generation times can be obtained. 
may not have been completely representative of the wider population. Exploring

547 heterogeneity in the generation time distribution between individuals and/or households with

548 different characteristics is an important topic for future work. This could involve, but is not

549 limited to, estimating the generation time distribution for individuals of different age, sex,

550 ethnicity and socio-economic status. Nonetheless, as well as providing updated SARS-CoV-2

551 generation time estimates, our study demonstrates that changes in the generation time can be

552 detected using data from household studies. Our finding that the generation time has become shorter highlights both the importance of continued monitoring of the generation time and the role of household studies in such monitoring efforts, particularly in light of the more recent emergence of novel SARS-CoV-2 variants.

556

557

In summary, we have inferred the SARS-CoV-2 generation time distribution in the UK using household data and two different transmission models. A key output of this research is one of the first estimates of the SARS-CoV-2 generation time outside Asia. Another crucial feature of our analysis is that it was based on data from beyond the first few months of the pandemic. Since this research suggests that the generation time may be changing, continued data collection and analysis is of clear importance.

563

\section{MATERIALS AND METHODS}

565

$\underline{\text { Data }}$

566 Data were obtained from a household study (Miller et al., 2021) conducted in 172 UK households (with 603 household members in total) by PHE between March and November 2020 (Figure 1-Source Data 1). In each household, an index case was recruited following a positive PCR test. The following were then recorded for each household member:

- The timing and outcome of (up to) two subsequent PCR tests. 
577 In the study, all household members who tested positive in either a PCR or antibody test were

- The outcome of an antibody test (carried out for 541 individuals - $90 \%$ of the study cohort)

- Whether or not the household member developed symptoms.

- The date of symptom onset (only for symptomatic individuals with a positive PCR or antibody test).
578

579

580

581

582

583

584

585

586

587

588

589

590

591

592

593

594

595 assumed to have been infected. Conversely, all individuals who tested negative for antibodies and did not return a positive PCR test (i.e., the two PCR tests were either negative or were not carried out) were assumed to have remained uninfected, irrespective of symptom status. For 34 individuals (6\% of the study cohort), no antibody test was carried out and any PCR tests were negative. Since it is unknown whether or not these individuals were infected, they were excluded from our main analyses (but were counted in the household size), although we also considered the sensitivity of our results to this assumption.

In two households, at least one household member developed symptoms 55-56 days prior to the symptom onset date of the index case, with no other household members developing symptoms (or returning a positive PCR or antibody test) between these dates. In contrast, the maximum gap between successive symptom onset dates in the remaining households was 25 days (Figure 1-figure supplement 3). Data from these two households were excluded from our analyses, on the basis that the virus was most likely introduced multiple times into these households. Three other households were also excluded from our analyses because, other than the index cases in each household, all other household members were of unknown infection status (i.e., they were among the individuals for whom no antibody test was carried out and any PCR tests were negative). 
597 Overall, aside from the five excluded households, the 167 remaining households comprised

598587 individuals, of whom 330 became infected and developed symptoms, 27 became infected

599 but remained asymptomatic, 200 remained uninfected, and the remaining 30 were of

600 unknown infection status. The number of households and individuals recruited into the study

601 by month is shown in Figure 3-figure supplement 1.

602

603 Models

604 General modelling framework

605 Throughout, we denote the expected force of infection exerted by an infected host onto each 606 susceptible member of their household, at time $\tau$ since infection, by $\beta(\tau)$, where we assumed

$$
\beta(\tau)=\left(\beta_{0} / n\right) f(\tau)
$$

607 for a host who develops symptoms, and

$$
\beta(\tau)=\alpha_{A}\left(\beta_{0} / n\right) f(\tau)
$$

608 for a host who remains asymptomatic throughout infection. Here:

609

- $\beta_{0}$ is the overall infectiousness parameter, describing the expected number of

610

611

612

- $n$ is the household size. The scaling of $\beta(\tau)$ with $1 / n$ corresponds to frequency-

613

614 dependent transmission, as assumed by (Cauchemez et al., 2014, 2004), although we

615

616 household transmissions generated by a single infected host (who develops symptoms) in a large, otherwise entirely susceptible, household.

617 carried out a sensitivity analysis in which we considered alternative possibilities where household transmission is density-dependent (without the scaling factor $1 / n$ ), scales with $1 / n^{0.5}$ (Endo et al., 2019), or scales with $1 /(n-1)$.

- $f(\tau)$ is the generation time distribution. 
- $\alpha_{A}$ is the relative infectiousness of infected hosts who remain asymptomatic throughout infection. We assumed a value of 0.35 (Buitrago-Garcia et al., 2020) in most of our analyses, although we considered different values of $\alpha_{A}$ in a sensitivity analysis.

Except where otherwise stated, we considered the generation time distribution assuming a constant supply of susceptibles during infection, $f(\tau)$, which corresponds to the normalised expected infectiousness profile and gives a widely applicable generation time estimate (see Discussion). However, realised generation times within a household may be shorter than predicted by this distribution due to the depletion of susceptible household members before longer generation times can be attained (Cauchemez et al., 2009; Fraser, 2007; Park et al., 2020b). For example, if infected hosts are (on average) equally infectious at two times since infection, $\tau_{1}<\tau_{2}$, then $f\left(\tau_{1}\right)=f\left(\tau_{2}\right)$. However, because the number of susceptible household members may decrease between these two times (i.e., either the host under consideration, or another infected household member, may transmit the virus within the household in the intervening time), then transmission is in fact more likely to occur in a household at the earlier time, $\tau_{1}$, when more susceptibles are available. Therefore, we also predicted the mean and standard deviation of realised generation times within the study households in Figure 1-figure supplement 4.

We considered two different models of infectiousness, which are outlined below. Under each model, expressions were derived in (Hart et al., 2021) for the generation time, TOST and serial interval distributions, in addition to the proportion of transmissions occurring before symptom onset. These expressions are given in the Appendix (other than the generation time 
642 distribution and proportion of presymptomatic transmissions for the independent transmission 643 and symptoms model, which are stated below).

644

645 Independent transmission and symptoms model

646 In this model, the infectiousness of an infected host (who does not remain asymptomatic

647 throughout infection; asymptomatic infected hosts are considered separately) at a given time

648 since infection, $\tau$, is assumed to be independent of exactly when the host develops symptoms

649 - i.e., the generation time and incubation period are independent. In our main analyses using

650 this model, we assumed that the generation time distribution, $f(\tau)$, is the probability density

651 function of a lognormal distribution (Ferguson et al., 2005) (an alternative case of a gamma

652 distributed generation time is considered in Figure 1-figure supplement 6). The mean and

653 standard deviation of this distribution, in addition to $\beta_{0}$, were estimated when we fitted the

654 model to the household transmission data.

655

656 Under the assumption of independent transmission and symptoms, the proportion of transmissions occurring prior to symptom onset (among infectors who develop symptoms) is

658 given by (Ferretti et al., 2020b; Fraser et al., 2004)

$$
\int_{0}^{\infty} f(\tau)\left(1-F_{\text {inc }}(\tau)\right) \mathrm{d} \tau
$$

where $F_{\text {inc }}$ is the cumulative distribution function of the incubation period (this was assumed to be known; the exact incubation period distribution we used is given under "Parameter estimation" below).

662

Mechanistic model

664 Under the mechanistic model (Hart et al., 2021), infectors who develop symptoms progress

665 through independent latent $(E)$, presymptomatic infectious $(P)$ and symptomatic infectious $(I)$ 
stages of infection. We assumed the duration of each stage to be gamma distributed, and infectiousness was assumed to be constant during each stage. Under these assumptions, an expression can be derived for the expected infectiousness, $\beta\left(\tau \mid \tau_{\text {inc }}\right.$ ), of a host (who develops symptoms) at each time since infection $\tau$, conditional on their incubation period $\tau_{\text {inc }}$. We assumed that entirely asymptomatic infected hosts follow the same stage progression as those who develop symptoms, although in this case, the distinction between the $P$ and $I$ stages has no epidemiological meaning. Details of the mechanistic approach, including the formula for $\beta\left(\tau \mid \tau_{\text {inc }}\right)$, are provided in the Appendix.

When we fitted this model to the household transmission data, three model parameters were estimated in addition to $\beta_{0}$. These parameters correspond to:

- The ratio between the mean latent $(E)$ period and the mean incubation (combined $E$ and $P$ ) period (where the latter was assumed to be known).

- The mean symptomatic infectious $(I)$ period.

- The ratio between the transmission rates when potential infectors are in the $P$ and $I$ stages.

\section{$\underline{\text { Likelihood function }}$}

Here, we consider a household of size $n$, in which $n_{I}$ household members become infected (of whom $n_{S}$ develop symptoms and $n_{A}$ remain asymptomatic throughout infection) and $n_{U}=n-n_{I}$ remain uninfected. We derive an expression for the likelihood of the parameters of either model of infectiousness, given the entire sequence of infection times of individuals in the household $\left(t_{1}<\cdots<t_{n_{I}}\right)$ as well as the precise symptom onset time $\left(t_{s, j}\right)$ of each host, $j$, who develops symptoms. In the case of the mechanistic model, the likelihood also depends on the times at which entirely asymptomatic infected hosts enter the $I$ stage of infection 
(these times are also denoted by $t_{s, j}$, although for asymptomatic infected individuals these

times have no epidemiological meaning). Since exact infection times were not available

693 within study households, and it was unknown exactly when each symptomatic infected host

694 developed symptoms within their recorded symptom onset date, we used data augmentation

695

MCMC to fit the two models to the UK household transmission data using this likelihood

696 function (see further details below).

697

698 When deriving the likelihood, we made several simplifying assumptions:

- The virus is introduced once into the household (i.e., no subsequent infections from the community occur following the infection of the primary case).

- No co-primary cases (we relaxed this assumption in the Appendix, Figure 1-figure supplement 5 and Figure 3-figure supplement 6).

- Potential bias towards more recent introduction of the virus into the household if

706

707

We denote the expected infectiousness of household member $j$, at time $\tau$ since infection, by $\beta_{j}(\tau)$. For the mechanistic model in which transmission and symptoms are not independent, this infectiousness is conditional on the duration of the incubation period, $t_{s, j}-t_{j}$, for a host who develops symptoms (the infectiousness is also conditional on $\left(t_{s, j}-t_{j}\right)$ for an entirely asymptomatic infected host, although this interval has no epidemiological meaning for such

712 individuals). The total (instantaneous) force of infection exerted at time $t$ on each susceptible 713 household member is then 


$$
\lambda(t)=\sum_{j=1}^{n_{I}} \beta_{j}\left(t-t_{j}\right)
$$

714 where $\beta_{j}\left(t-t_{j}\right)=0$ for $t \leq t_{j}$, and the cumulative force of infection is

$$
\Lambda(t)=\int_{-\infty}^{t} \lambda(s) \mathrm{d} s
$$

715

716 For $k=2, \ldots, n_{I}$, conditional on the sequence of infection times up to time $t_{k}$, the probability

717 that host $k$ becomes infected at time $t_{k}$ is given by

$$
\lambda\left(t_{k}\right) \exp \left(-\Lambda\left(t_{k}\right)\right)
$$

718 where $\exp \left(-\Lambda\left(t_{k}\right)\right)$ represents the probability of host $k$ avoiding infection from household

719 contacts that occurred before their actual time of infection, $t_{k}$ (Cauchemez et al., 2004;

720 Ferguson et al., 2005). This factor, which was not included in the likelihood when we

721 previously estimated the generation time using data from infector-infectee transmission pairs

722 (Hart et al., 2021), is required here because of the regularity of household contacts. Since

723 household contacts occur frequently, it is necessary to account explicitly for contacts between

724 infected and susceptible individuals that did not lead to transmission. The inclusion of this

725 factor in the likelihood therefore corrects for the regularity of household contacts to ensure

726 widely applicable generation time estimates (note that this factor is equal to one in the limit

727 of a very small overall household infectiousness parameter, $\beta_{0}$ ).

728

729 For $k=n_{I}+1, \ldots, n$, conditional on the entire sequence of infection times, $t_{1}, \ldots, t_{n_{I}}$, the

730 probability of host $k$ never being infected is given by $\exp (-\Lambda(\infty))$. In the case of

731 independent transmission and symptoms, we have

$$
\left.\exp (-\Lambda(\infty))=\exp \left(-\beta_{0}\left(n_{S}+\alpha_{A} n_{A}\right) / n\right)\right)
$$


whereas for the mechanistic model, $\exp (-\Lambda(\infty))$ instead depends on the incubation periods asymptomatic infected hosts (see the Appendix).

735

736 The likelihood contribution from the household, $L(\theta)$, where $\theta$ is the vector of unknown model parameters, can therefore be written as

$$
L(\theta)=\prod_{k=1}^{n} L_{k, 1}(\theta) L_{k, 2}(\theta)
$$

738 Here, $L_{k, 1}(\theta)$ is the contribution to the likelihood from the transmission, or absence of 739 transmission, to host $k$, i.e.,

$$
L_{k, 1}(\theta)=\left\{\begin{aligned}
1, & \text { for } k=1 ; \\
\lambda\left(t_{k}\right) \exp \left(-\Lambda\left(t_{k}\right)\right), & \text { for } k=2, \ldots, n_{I} \\
\exp (-\Lambda(\infty)), & \text { for } k=n_{I}+1, \ldots, n
\end{aligned}\right.
$$

$740 L_{k, 2}(\theta)$ is the contribution from the incubation period of host $k$ (where applicable), i.e., for

741 the independent transmission and symptoms model,

$$
L_{k, 2}(\theta)=\left\{\begin{aligned}
f_{\text {inc }}\left(t_{s, k}-t_{k}\right), & \text { if host } k \text { becomes infected and develops symptoms; } \\
1, & \text { otherwise; }
\end{aligned}\right.
$$

742 where $f_{\text {inc }}$ is the probability density function of the incubation period (this was

743 assumed to be known; the exact incubation period distribution we used is given below).

744 For the mechanistic model, we also have a contribution to the likelihood from the (in

745 this case not epidemiologically meaningful) times $\left(t_{s, k}-t_{k}\right)$ for entirely asymptomatic

746 infected hosts, so that

$$
L_{k, 2}(\theta)=\left\{\begin{aligned}
f_{\text {inc }}\left(t_{s, k}-t_{k}\right), & \text { for } k=1, \ldots, n_{I} \\
1, & \text { for } k=n_{I}+1, \ldots, n
\end{aligned}\right.
$$

$748 \quad$ Parameter estimation

Incubation period 
For the independent transmission and symptoms model, we assumed a lognormal incubation

751

752

753

754

755

756

757

758

759

760

761

762

763

764

765

766

767

768

769

770

771

772

773

774 period distribution with mean 5.8 days and standard deviation 3.1 days (McAloon et al., 2020). For the mechanistic model, we assumed a gamma distributed incubation period with the same mean and standard deviation; this was for mathematical convenience, since the incubation period could then be decomposed into the sum of independent gamma distributed latent and presymptomatic infectious periods. Results for the independent transmission and symptoms model using a gamma distributed incubation period are shown in Figure 1-figure supplement 7 , and uncertainty in the exact parameters of the incubation period distribution is accounted for in Figure 1-figure supplement 8.

\section{Parameter fitting procedure}

Unknown model parameters were estimated using data augmentation MCMC. The observed data comprised information about whether or not individuals were ever infected and/or displayed symptoms, symptom onset dates, and for some individuals an upper bound on their infection time (corresponding to the date of a positive PCR test). These data were augmented with precise times of infection and symptom onset (where applicable) for each infected host. No prior assumptions were made about the order of transmissions within the household.

Below, we outline the parameter fitting procedure that we used for the independent transmission and symptoms model. The procedure used for the mechanistic model was similar and is described in the Appendix.

Lognormal priors were assumed for fitted model parameters (these parameters were the mean and standard deviation of the generation time distribution, in addition to the overall infectiousness, $\beta_{0}$ ). The priors for the mean and standard deviation of the generation time 
distribution had medians of 5 days and 2 days, respectively (these choices were informed by

776

777

778

779

780

781

Here, we denote the vector of model parameters by $\theta$, and the augmented data by

$$
\boldsymbol{t}=\left(\boldsymbol{t}^{(1)}, \ldots, \boldsymbol{t}^{(M)}\right),
$$

782

783

number of households. We write the (overall) likelihood as

$$
L(\theta ; \boldsymbol{t})=\prod_{m=1}^{M} L^{(m)}\left(\theta ; \boldsymbol{t}^{(m)}\right),
$$

784

where the likelihood contribution, $L^{(m)}\left(\theta ; \boldsymbol{t}^{(m)}\right)$, from each household, $m$, was computed as described in the previous section (i.e., all households in the study were assumed to be 786 independent), and we denote the prior density of $\theta$ by $\pi(\theta)$.

787

788

In each step of the chain, we carried out (in turn) one of the following:

1. Propose new values for each entry of the vector of model parameters, $\theta$, using independent normal proposal distributions for each parameter (around the corresponding parameter values in the previous step of the chain). Accept the proposed parameters, $\theta_{\text {prop }}$, with probability

$$
\min \left(\frac{L\left(\theta_{\text {prop }} ; \boldsymbol{t}\right) \pi\left(\theta_{\text {prop }}\right)}{L\left(\theta_{\text {old }} ; \boldsymbol{t}\right) \pi\left(\theta_{\text {old }}\right)}, 1\right)
$$

where $\theta_{\text {old }}$ denotes the vector of parameter values from the previous step of the chain, and where the augmented data, $\boldsymbol{t}$, remain unchanged in this step. 
2. Propose new values for the precise symptom onset times of each symptomatic infected host, using independent uniform proposal distributions (within the day of symptom of onset for each host). For each household, $m$, accept the proposed augmented data, $\boldsymbol{t}_{\text {prop }}^{(m)}$, from that household with probability

$$
\min \left(\frac{L^{(m)}\left(\theta ; \boldsymbol{t}_{\text {prop }}^{(m)}\right)}{L^{(m)}\left(\theta ; \boldsymbol{t}_{\text {old }}^{(m)}\right)}, 1\right)
$$

where $\boldsymbol{t}_{\text {old }}^{(m)}$ denotes the corresponding augmented data from the previous step of the chain, and where the model parameters, $\theta$, remain unchanged in this step (i.e., proposed times are accepted/rejected independently for each household, according to the likelihood contribution from that household).

3. Propose new values for the infection time of one randomly chosen symptomatic infected host in each household (in households where there was at least one), using independent normal proposal distributions (around the equivalent times in the previous step of the chain). For each household, $m$, accept the proposed augmented data, $\boldsymbol{t}_{\text {prop }}^{(m)}$, from that household with probability

$$
\min \left(\frac{L^{(m)}\left(\theta ; \boldsymbol{t}_{\text {prop }}^{(m)}\right)}{L^{(m)}\left(\theta ; \boldsymbol{t}_{\text {old }}^{(m)}\right)}, 1\right)
$$

4. Propose new values for the infection time of one randomly chosen asymptomatic infected host in each household (in households where there was at least one), using independent normal proposal distributions (around the equivalent times in the previous step of the chain). For each household, $m$, accept the proposed augmented data, $\boldsymbol{t}_{\text {prop }}^{(m)}$, from that household with probability 


$$
\min \left(\frac{L^{(m)}\left(\theta ; \boldsymbol{t}_{\text {prop }}^{(m)}\right)}{L^{(m)}\left(\theta ; \boldsymbol{t}_{\text {old }}^{(m)}\right)}, 1\right)
$$

815

816 The chain was run for $10,000,000$ iterations; the first 2,000,000 iterations were discarded as

817 burn-in. Posteriors were obtained by recording every 100 iterations of the chain.

818 
The household study was approved by the PHE Research Ethics and Governance Group as part of the portfolio of PHE's enhanced surveillance activities in response to the pandemic.

822

\section{Acknowledgments:}

824 Thanks to Pauline Waight, who managed the data for the household study, and to the PHE

825 staff who collected the data and tested the PCR and serum samples. Thanks also to Rob

826 Challen, Julia Gog, Matt Keeling and other members of the Juniper Consortium

827 (www.maths.org/juniper/) for helpful comments about this research.

828

Competing interests:

AE received a research grant from Taisho Pharmaceutical Co., Ltd. All the other authors declare no competing interests.

\section{References:}

Abbott S, Hellewell J, Thompson RN, Sherratt K, Gibbs HP, Bosse NI, Munday JD, Meakin S, Doughty EL, Chun JY, Chan Y-WD, Finger F, Campbell P, Endo A, Pearson CAB, Gimma A, Russell T, CMMID COVID modelling group, Flasche S, Kucharski AJ, Eggo RM, Funk S. 2020. Estimating the time-varying reproduction number of SARSCoV-2 using national and subnational case counts. Wellcome Open Res 5:112. doi:10.12688/wellcomeopenres.16006.2

Ali ST, Wang L, Lau EHY, Xu X-K, Du Z, Wu Y, Leung GM, Cowling BJ. 2020. Serial interval of SARS-CoV-2 was shortened over time by nonpharmaceutical interventions. Science 369:1106-1109. doi:10.1126/science.abc9004

Anderson RM, May RM. 1992. Infectious Diseases of Humans: Dynamics and Control. OUP Oxford.

Ashcroft P, Huisman JS, Lehtinen S, Bouman JA, Althaus CL, Regoes RR, Bonhoeffer S. 2020. COVID-19 infectivity profile correction. Swiss Med Wkly 150:w20336. doi:10.4414/smw.2020.20336

Ashcroft P, Lehtinen S, Angst DC, Low N, Bonhoeffer S. 2021. Quantifying the impact of quarantine duration on COVID-19 transmission. eLife 10:e63704.

850 doi:10.7554/eLife.63704 
868

869

870

871

872

873

874

875

876

877

878

879

880

881

882

883

884

885

886

887

888

889

890

891

892

893

894

895

896

897

898

899

900

901

902

903

904
Bacallado S, Zhao Q, Ju N. 2020. Letter to the editor: Generation interval for COVID-19 based on symptom onset data. Eurosurveillance 25:2001381. doi:10.2807/15607917.ES.2020.25.29.2001381

Boddington NL, Charlett A, Elgohari S, Byers C, Coughlan L, Vilaplana TG, Whillock R, Sinnathamby M, Panagiotopoulos N, Letley L, MacDonald P, Vivancos R, Edeghere O, Shingleton J, Bennett E, Cottrell S, McMenamin J, Zambon M, Ramsay M, Dabrera G, Saliba V, Bernal JL. 2021. Epidemiological and clinical characteristics of early COVID-19 cases, United Kingdom of Great Britain and Northern Ireland. Bull World Health Organ 99:178-189. doi:10.2471/BLT.20.265603

Britton T, Scalia Tomba G. 2019. Estimation in emerging epidemics: biases and remedies. $J$ $R$ Soc Interface 16:20180670. doi:10.1098/rsif.2018.0670

Buitrago-Garcia D, Egli-Gany D, Counotte MJ, Hossmann S, Imeri H, Ipekci AM, Salanti G, Low N. 2020. Occurrence and transmission potential of asymptomatic and presymptomatic SARS-CoV-2 infections: A living systematic review and metaanalysis. PLOS Med 17:e1003346. doi:10.1371/journal.pmed.1003346

Bushman M, Worby C, Chang H-H, Kraemer MUG, Hanage WP. 2021. Transmission of SARS-CoV-2 before and after symptom onset: impact of nonpharmaceutical interventions in China. Eur J Epidemiol 36:429-439. doi:10.1007/s10654-021-007464

Casey-Bryars M, Griffin J, McAloon C, Byrne A, Madden J, Evoy DM, Collins Á, Hunt K, Barber A, Butler F, Lane EA, O'Brien K, Wall P, Walsh K, More SJ. 2021.

Presymptomatic transmission of SARS-CoV-2 infection: a secondary analysis using published data. BMJ Open 11:e041240. doi:10.1136/bmjopen-2020-041240

Cauchemez S, Carrat F, Viboud C, Valleron AJ, Boëlle PY. 2004. A Bayesian MCMC approach to study transmission of influenza: application to household longitudinal data. Stat Med 23:3469-3487. doi:10.1002/sim.1912

Cauchemez S, Donnelly CA, Reed C, Ghani AC, Fraser C, Kent CK, Finelli L, Ferguson NM. 2009. Household transmission of 2009 pandemic influenza $A$ (H1N1) virus in the United States. N Engl J Med 361:2619-2627. doi:10.1056/NEJMoa0905498

Cauchemez S, Ferguson NM, Fox A, Mai LQ, Thanh LT, Thai PQ, Thoang DD, Duong TN, Hoa LNM, Hien NT, Horby P. 2014. Determinants of influenza transmission in South East Asia: insights from a household cohort study in Vietnam. PLOS Pathog 10:e1004310. doi:10.1371/journal.ppat.1004310

Challen R, Brooks-Pollock E, Tsaneva-Atanasova K, Danon L. 2021. Meta-analysis of the severe acute respiratory syndrome coronavirus 2 serial intervals and the impact of parameter uncertainty on the coronavirus disease 2019 reproduction number. Stat Methods Med Res 09622802211065159. doi:10.1177/09622802211065159

Cheng H-Y, Jian S-W, Liu D-P, Ng T-C, Huang W-T, Lin H-H, for the Taiwan COVID-19 Outbreak Investigation Team. 2020. Contact tracing assessment of COVID-19 transmission dynamics in Taiwan and risk at different exposure periods before and after symptom onset. JAMA Intern Med 180:1156. doi:10.1001/jamainternmed.2020.2020

Davies NG, Abbott S, Barnard RC, Jarvis CI, Kucharski AJ, Munday JD, Pearson CAB, Russell TW, Tully DC, Washburne AD, Wenseleers T, Gimma A, Waites W, Wong KLM, Zandvoort K van, Silverman JD, Group1 1 CC-19 W, Consortium $\ddagger$ C-19 GU (COG-U, Diaz-Ordaz K, Keogh R, Eggo RM, Funk S, Jit M, Atkins KE, Edmunds WJ. 2021. Estimated transmissibility and impact of SARS-CoV-2 lineage B.1.1.7 in England. Science eabg3055. doi:10.1126/science.abg3055

Deng Y, You C, Liu Y, Qin J, Zhou X-H. 2020. Estimation of incubation period and generation time based on observed length-biased epidemic cohort with censoring for COVID-19 outbreak in China. Biometrics 1-13. doi:https://doi.org/10.1111/biom.13325

Diekmann O, Heesterbeek JAP. 2000. Mathematical Epidemiology of Infectious Diseases: Model Building, Analysis and Interpretation. John Wiley \& Sons. 
Endo A, Uchida M, Kucharski AJ, Funk S. 2019. Fine-scale family structure shapes influenza transmission risk in households: Insights from primary schools in Matsumoto city, 2014/15. PLOS Comput Biol 15:e1007589. doi:10.1371/journal.pcbi.1007589

Ferguson NM, Cummings DAT, Cauchemez S, Fraser C, Riley S, Meeyai A, lamsirithaworn S, Burke DS. 2005. Strategies for containing an emerging influenza pandemic in Southeast Asia. Nature 437:209-214. doi:10.1038/nature04017

Ferretti L, Ledda A, Wymant C, Zhao L, Ledda V, Abeler L, Kendall M, Nurtay A, Cheng $\mathrm{H}-\mathrm{Y}$, $\mathrm{Ng} \mathrm{T-C}$, Lin H-H, Hinch R, Masel J, Kilpatrick AM, Fraser C. 2020a. The timing of COVID-19 transmission. medRxiv 2020.09.04.20188516. doi:https://doi.org/10.1101/2020.09.04.20188516

Ferretti L, Wymant C, Kendall M, Zhao L, Nurtay A, Abeler-Dörner L, Parker M, Bonsall D, Fraser C. 2020b. Quantifying SARS-CoV-2 transmission suggests epidemic control with digital contact tracing. Science 368:eabb6936. doi:10.1126/science.abb6936

Fraser C. 2007. Estimating Individual and Household Reproduction Numbers in an Emerging Epidemic. PLOS ONE 2:e758. doi:10.1371/journal.pone.0000758

Fraser C, Riley S, Anderson RM, Ferguson NM. 2004. Factors that make an infectious disease outbreak controllable. Proc Natl Acad Sci 101:6146-6151. doi:10.1073/pnas.0307506101

Ganyani T, Kremer C, Chen D, Torneri A, Faes C, Wallinga J, Hens N. 2020. Estimating the generation interval for coronavirus disease (COVID-19) based on symptom onset data, March 2020. Eurosurveillance 25:2000257. doi:10.2807/15607917.ES.2020.25.17.2000257

Gostic KM, McGough L, Baskerville EB, Abbott S, Joshi K, Tedijanto C, Kahn R, Niehus R, Hay JA, Salazar PMD, Hellewell J, Meakin S, Munday JD, Bosse NI, Sherrat K, Thompson RN, White LF, Huisman JS, Scire J, Bonhoeffer S, Stadler T, Wallinga J, Funk S, Lipsitch M, Cobey S. 2020. Practical considerations for measuring the effective reproductive number, Rt. PLOS Comput Biol 16:e1008409. doi:10.1371/journal.pcbi.1008409

Griffin J, Casey M, Collins A, Hunt K, McEvoy D, Byrne A, McAloon C, Barber A, Lane EA, More S. 2020. Rapid review of available evidence on the serial interval and generation time of COVID-19. BMJ Open 10:e040263. doi:10.1136/bmjopen-2020040263

Hart WS, Maini PK, Thompson RN. 2021. High infectiousness immediately before COVID-19 symptom onset highlights the importance of continued contact tracing. eLife 10:e65534. doi:10.7554/eLife.65534

He X, Lau EHY, Wu P, Deng X, Wang J, Hao X, Lau YC, Wong JY, Guan Y, Tan X, Mo X, Chen Y, Liao B, Chen W, Hu F, Zhang Q, Zhong M, Wu Y, Zhao L, Zhang F, Cowling BJ, Li F, Leung GM. 2020. Temporal dynamics in viral shedding and transmissibility of COVID-19. Nat Med 26:672-675. doi:10.1038/s41591-020-0869-5

Hu S, Wang W, Wang Y, Litvinova M, Luo K, Ren L, Sun Q, Chen Xinghui, Zeng G, Li J, Liang L, Deng Z, Zheng W, Li M, Yang H, Guo J, Wang K, Chen Xinhua, Liu Z, Yan $H$, Shi H, Chen Z, Zhou Y, Sun K, Vespignani A, Viboud C, Gao L, Ajelli M, Yu H. 2021. Infectivity, susceptibility, and risk factors associated with SARS-CoV-2 transmission under intensive contact tracing in Hunan, China. Nat Commun 12:1533. doi:10.1038/s41467-021-21710-6

Knight J, Mishra S. 2020. Estimating effective reproduction number using generation time versus serial interval, with application to covid-19 in the Greater Toronto Area, Canada. Infect Dis Model 5:889-896. doi:10.1016/j.idm.2020.10.009

Knock ES, Whittles LK, Lees JA, Perez-Guzman PN, Verity R, FitzJohn RG, Gaythorpe KAM, Imai N, Hinsley W, Okell LC, Rosello A, Kantas N, Walters CE, Bhatia S, Watson OJ, Whittaker C, Cattarino L, Boonyasiri A, Djaafara BA, Fraser K, Fu H, Wang H, Xi X, Donnelly CA, Jauneikaite E, Laydon DJ, White PJ, Ghani AC, Ferguson NM, Cori A, Baguelin M. 2021. Key epidemiological drivers and impact of interventions in the 2020 SARS-CoV-2 epidemic in England. Sci Trans/ Med. doi:10.1126/scitranslmed.abg4262 
Lehtinen S, Ashcroft P, Bonhoeffer S. 2021. On the relationship between serial interval, infectiousness profile and generation time. J R Soc Interface 18:20200756. doi:10.1098/rsif.2020.0756

Manfredi P, D'Onofrio A, editors. 2013. Modeling the Interplay Between Human Behavior and the Spread of Infectious Diseases. New York: Springer-Verlag.

McAloon C, Collins Á, Hunt K, Barber A, Byrne AW, Butler F, Casey M, Griffin J, Lane E, McEvoy D, Wall P, Green M, O'Grady L, More SJ. 2020. Incubation period of COVID19: a rapid systematic review and meta-analysis of observational research. $B M J$ Open 10:e039652. doi:10.1136/bmjopen-2020-039652

Miller E, Waight PA, Andrews NJ, McOwat K, Brown KE, Höschler K, ljaz S, Letley L, Haskins D, Sinnathamby M, Cuthbertson $H$, Hallis B, Parimalanathan V, Lusignan S de, Lopez-Bernal J. 2021. Transmission of SARS-CoV-2 in the household setting: A prospective cohort study in children and adults in England. J Infect 83:483-489. doi:10.1016/j.jinf.2021.07.037

Parag KV, Thompson RN, Donnelly CA. 2021. Are epidemic growth rates more informative than reproduction numbers? Royal Statistical Society.

Park SW, Bolker BM, Champredon D, Earn DJD, Li M, Weitz JS, Grenfell BT, Dushoff J. 2020a. Reconciling early-outbreak estimates of the basic reproductive number and its uncertainty: framework and applications to the novel coronavirus (SARS-CoV-2) outbreak. J R Soc Interface 17:20200144. doi:10.1098/rsif.2020.0144

Park SW, Bolker BM, Funk S, Metcalf CJE, Weitz JS, Grenfell BT, Dushoff J. 2021. Roles of generation-interval distributions in shaping relative epidemic strength, speed, and control of new SARS-CoV-2 variants. medRxiv 2021.05.03.21256545. doi:10.1101/2021.05.03.21256545

Park SW, Champredon D, Dushoff J. 2020b. Inferring generation-interval distributions from contact-tracing data. J R Soc Interface 17:20190719. doi:10.1098/rsif.2019.0719

Pouwels KB, House T, Pritchard E, Robotham JV, Birrell PJ, Gelman A, Vihta K-D, Bowers N, Boreham I, Thomas H, Lewis J, Bell I, Bell JI, Newton JN, Farrar J, Diamond I, Benton P, Walker Ann Sarah, Pouwels KB, Walker A. Sarah, Crook D, Matthews PC, Peto T, Pritchard E, Stoesser N, Vihta K-D, Howarth A, Doherty G, Kavanagh J, Chau KK, Hatch SB, Ebner D, Martins Ferreira L, Christott T, Marsden BD, Dejnirattisai W, Mongkolsapaya J, Hoosdally S, Cornall R, Stuart DI, Screaton G, Eyre D, Bell J, Cox S, Paddon K, James T, House T, Newton JN, Robotham JV, Birrell P, Jordan H, Sheppard T, Athey G, Moody D, Curry L, Brereton P, Hay J, Vansteenhouse H, Bell I, Diamond I, Lambert A, Benton P, Rourke E, Hawkes S, Henry S, Scruton J, Stokes P, Thomas T, Allen J, Black R, Bovill H, Braunholtz D, Brown D, Collyer S, Crees M, Daglish C, Davies B, Donnarumma H, Douglas-Mann J, Felton A, Finselbach H, Fordham E, Ipser A, Jenkins J, Jones J, Kent K, Kerai G, Lloyd L, Masding V, Osborn E, Patel A, Pereira E, Pett T, Randall M, Reeve D, Shah P, Snook R, Studley R, Sutherland E, Swinn E, Thomas H, Tudor A, Weston J, Leib S, Tierney J, Farkas G, Cobb R, Van Galen F, Compton L, Irving J, Clarke J, Mullis R, Ireland L, Airimitoaie D, Nash C, Cox D, Fisher S, Moore Z, McLean J, Kerby M. 2021. Community prevalence of SARS-CoV-2 in England from April to November, 2020: results from the ONS Coronavirus Infection Survey. Lancet Public Health 6:e30-e38. doi:10.1016/S2468-2667(20)30282-6

Public Health England. 2021. SARS-CoV-2 variants of concern and variants under investigation in England (Technical briefing 7).

Public Health England. 2020. "The First Few Hundred (FF100)" Enhanced Case and Contact Protocol v12.

Sun K, Wang W, Gao L, Wang Y, Luo K, Ren L, Zhan Z, Chen X, Zhao S, Huang Y, Sun Q, Liu Z, Litvinova M, Vespignani A, Ajelli M, Viboud C, Yu H. 2021. Transmission heterogeneities, kinetics, and controllability of SARS-CoV-2. Science 371:eabe2424. doi:10.1126/science.abe2424

Svensson Å. 2007. A note on generation times in epidemic models. Math Biosci 208:300311. doi:10.1016/j.mbs.2006.10.010 
Thompson RN, Hollingsworth TD, Isham V, Arribas-Bel D, Ashby B, Britton T, Challenor P, Chappell LHK, Clapham H, Cunniffe NJ, Dawid AP, Donnelly CA, Eggo RM, Funk S, Gilbert N, Glendinning P, Gog JR, Hart WS, Heesterbeek H, House T, Keeling M, Kiss IZ, Kretzschmar ME, Lloyd AL, McBryde ES, McCaw JM, McKinley TJ, Miller JC, Morris M, O'Neill PD, Parag KV, Pearson CAB, Pellis L, Pulliam JRC, Ross JV, Tomba GS, Silverman BW, Struchiner CJ, Tildesley MJ, Trapman P, Webb CR, Mollison D, Restif O. 2020. Key questions for modelling COVID-19 exit strategies. Proc $R$ Soc B Biol Sci 287:20201405. doi:10.1098/rspb.2020.1405

Tindale LC, Stockdale JE, Coombe M, Garlock ES, Lau WYV, Saraswat M, Zhang L, Chen D, Wallinga J, Colijn C. 2020. Evidence for transmission of COVID-19 prior to symptom onset. eLife 9:e57149. doi:10.7554/eLife.57149

Vöhringer HS, Sanderson T, Sinnott M, De Maio N, Nguyen T, Goater R, Schwach F, Harrison I, Hellewell J, Ariani CV, Gonçalves S, Jackson DK, Johnston I, Jung AW, Saint C, Sillitoe J, Suciu M, Goldman N, Panovska-Griffiths J, Birney E, Volz E, Funk S, Kwiatkowski D, Chand M, Martincorena I, Barrett JC, Gerstung M. 2021. Genomic reconstruction of the SARS-CoV-2 epidemic in England. Nature 600:506-511. doi:10.1038/s41586-021-04069-y

Volz E, Mishra S, Chand M, Barrett JC, Johnson R, Geidelberg L, Hinsley WR, Laydon DJ, Dabrera G, O'Toole Á, Amato R, Ragonnet-Cronin M, Harrison I, Jackson B, Ariani CV, Boyd O, Loman NJ, McCrone JT, Gonçalves S, Jorgensen D, Myers R, Hill V, Jackson DK, Gaythorpe K, Groves N, Sillitoe J, Kwiatkowski DP, Flaxman S, Ratmann O, Bhatt S, Hopkins S, Gandy A, Rambaut A, Ferguson NM. 2021. Assessing transmissibility of SARS-CoV-2 lineage B.1.1.7 in England. Nature 593:266-269. doi:10.1038/s41586-021-03470-x

Wallinga J, Lipsitch M. 2007. How generation intervals shape the relationship between growth rates and reproductive numbers. Proc R Soc B Biol Sci 274:599-604. doi:10.1098/rspb.2006.3754

Wells CR, Townsend JP, Pandey A, Moghadas SM, Krieger G, Singer B, McDonald RH, Fitzpatrick MC, Galvani AP. 2021. Optimal COVID-19 quarantine and testing strategies. Nat Commun 12:356. doi:10.1038/s41467-020-20742-8

Xia W, Liao J, Li C, Li Yuanyuan, Qian X, Sun X, Xu H, Mahai G, Zhao X, Shi L, Liu J, Yu L, Wang $M$, Wang $Q$, Namat A, Li Ying, Qu J, Liu Q, Lin X, Cao S, Huan S, Xiao J, Ruan F, Wang $H, X u$ Q, Ding X, Fang X, Qiu F, Ma J, Zhang Y, Wang A, Xing Y, Xu S. 2020. Transmission of corona virus disease 2019 during the incubation period may lead to a quarantine loophole. medRxiv 2020.03.06.20031955. doi:10.1101/2020.03.06.20031955

Zhang J, Litvinova M, Wang W, Wang Y, Deng X, Chen Xinghui, Li M, Zheng W, Yi L, Chen Xinhua, Wu Q, Liang Y, Wang X, Yang J, Sun K, Longini IM, Halloran ME, Wu P, Cowling BJ, Merler S, Viboud C, Vespignani A, Ajelli M, Yu H. 2020. Evolving epidemiology and transmission dynamics of coronavirus disease 2019 outside Hubei province, China: a descriptive and modelling study. Lancet Infect Dis 20:793-802. doi:10.1016/S1473-3099(20)30230-9 
1059 Source Data:

1060 Figure 1-Source Data 1. Household transmission data. The transmission data from 172 households used in 1061 our analyses. 
Figure Supplements:
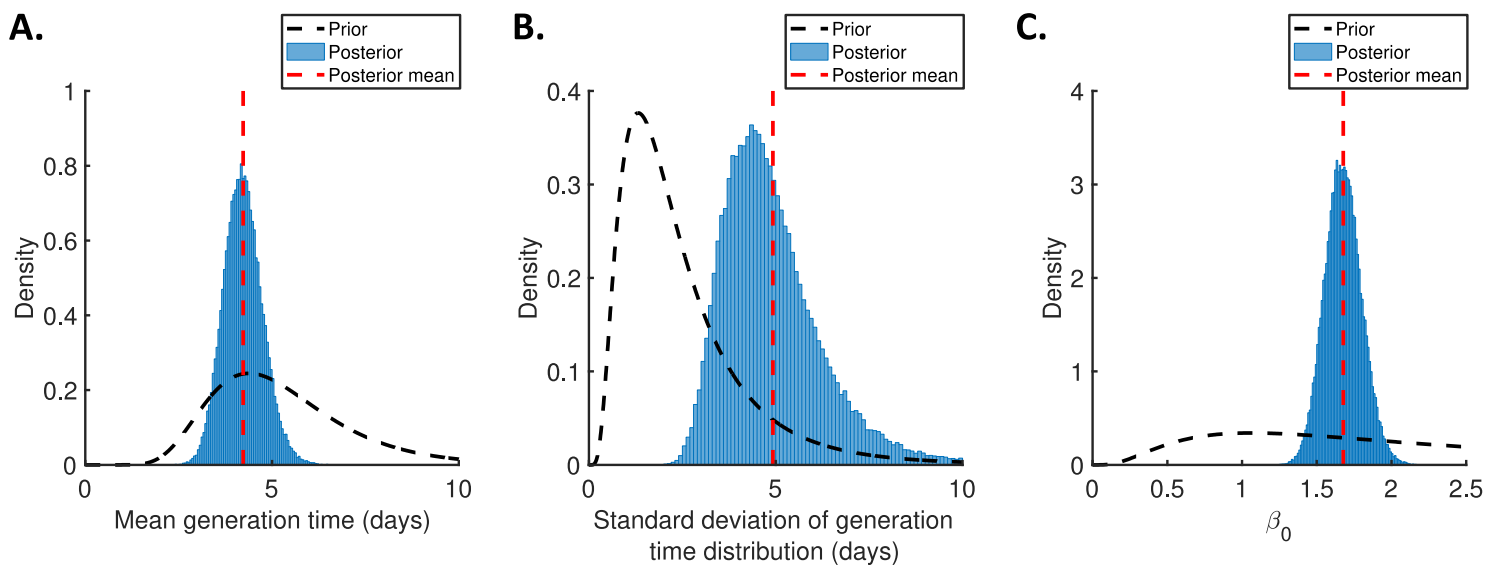

1064 Figure 1-figure supplement 1. Posterior distributions of fitted parameters for the independent

1065 transmission and symptoms model. Prior distributions (black dashed lines), posterior distributions (blue bars), and posterior means (vertical red dashed lines) of fitted parameters in the independent transmission and symptoms model. A. Mean generation time. B. Standard deviation of the generation time distribution. C. Overall infectiousness, $\beta_{0}$. 
A.

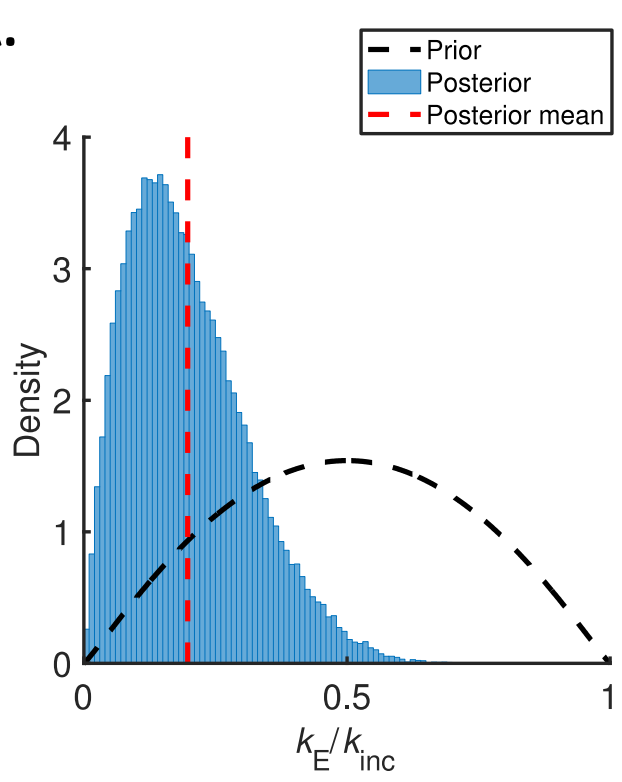

C.

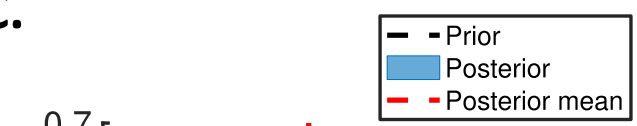

B.

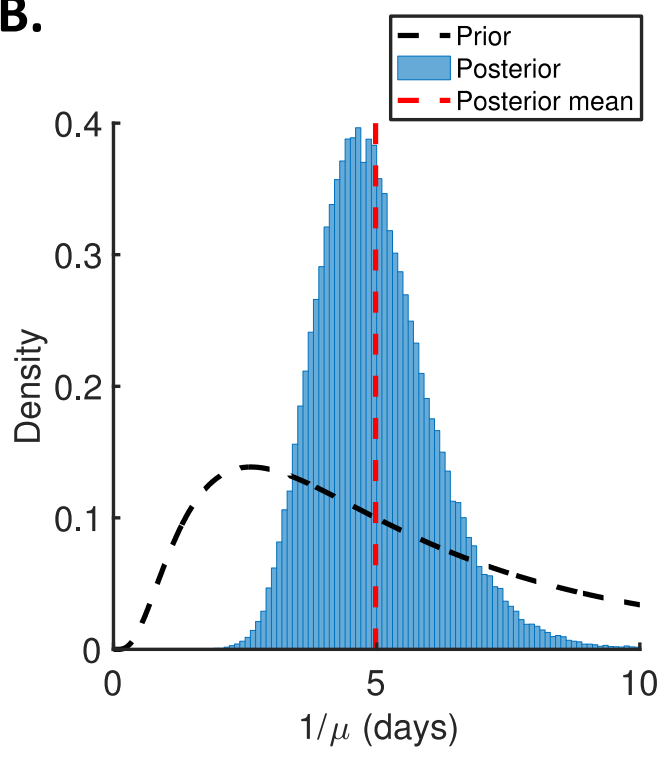

D.

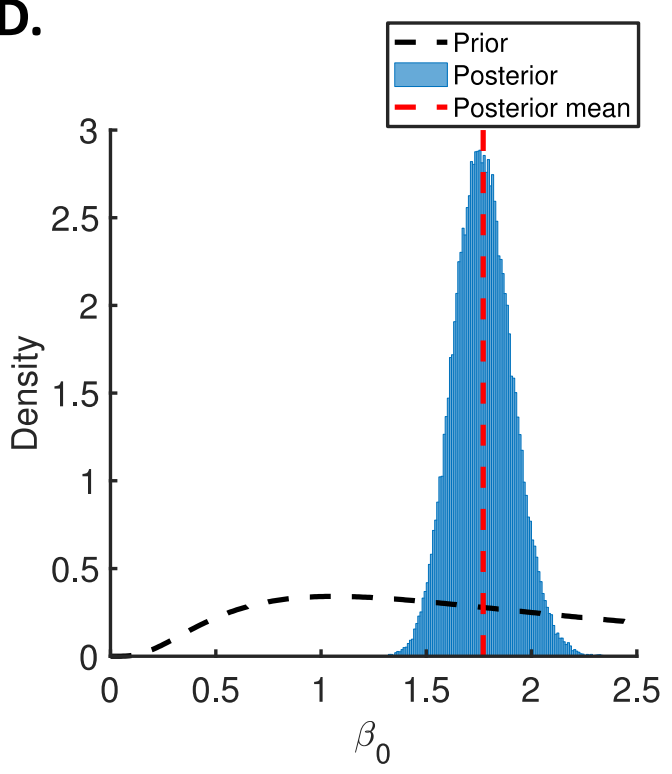

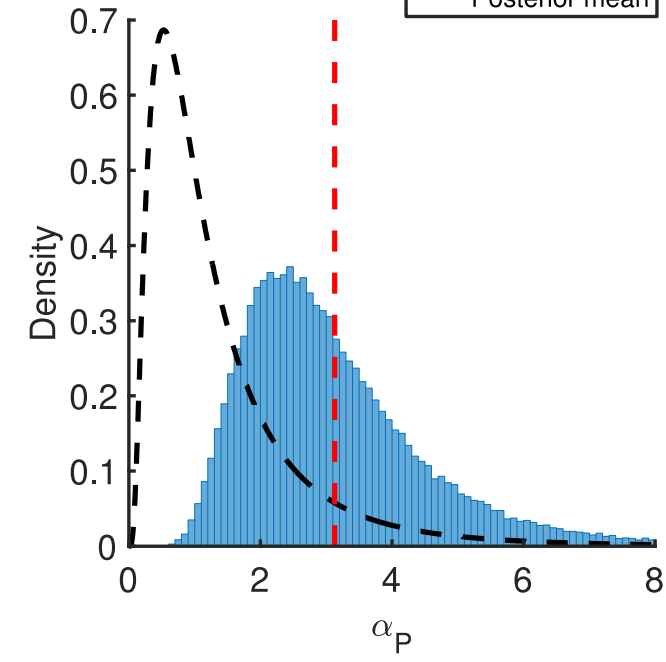

1071 Figure 1-figure supplement 2. Posterior distributions of fitted parameters for the mechanistic model. Prior

1072 distributions (black dashed lines), posterior distributions (blue bars), and posterior means (vertical red dashed

1073 lines) of fitted parameters in the mechanistic model. A. Ratio of mean durations of the latent $(E)$ and incubation

1074 (combined $E$ and $P$ ) periods, $k_{E} / k_{\text {inc }}$. B. Mean symptomatic infectious $(I)$ period, $1 / \mu$. C. Ratio of transmission

1075 rates in the presymptomatic infectious $(P)$ and symptomatic infectious $(I)$ stages of infection, $\alpha_{P}$. D. Overall

1076 infectiousness, $\beta_{0}$. 


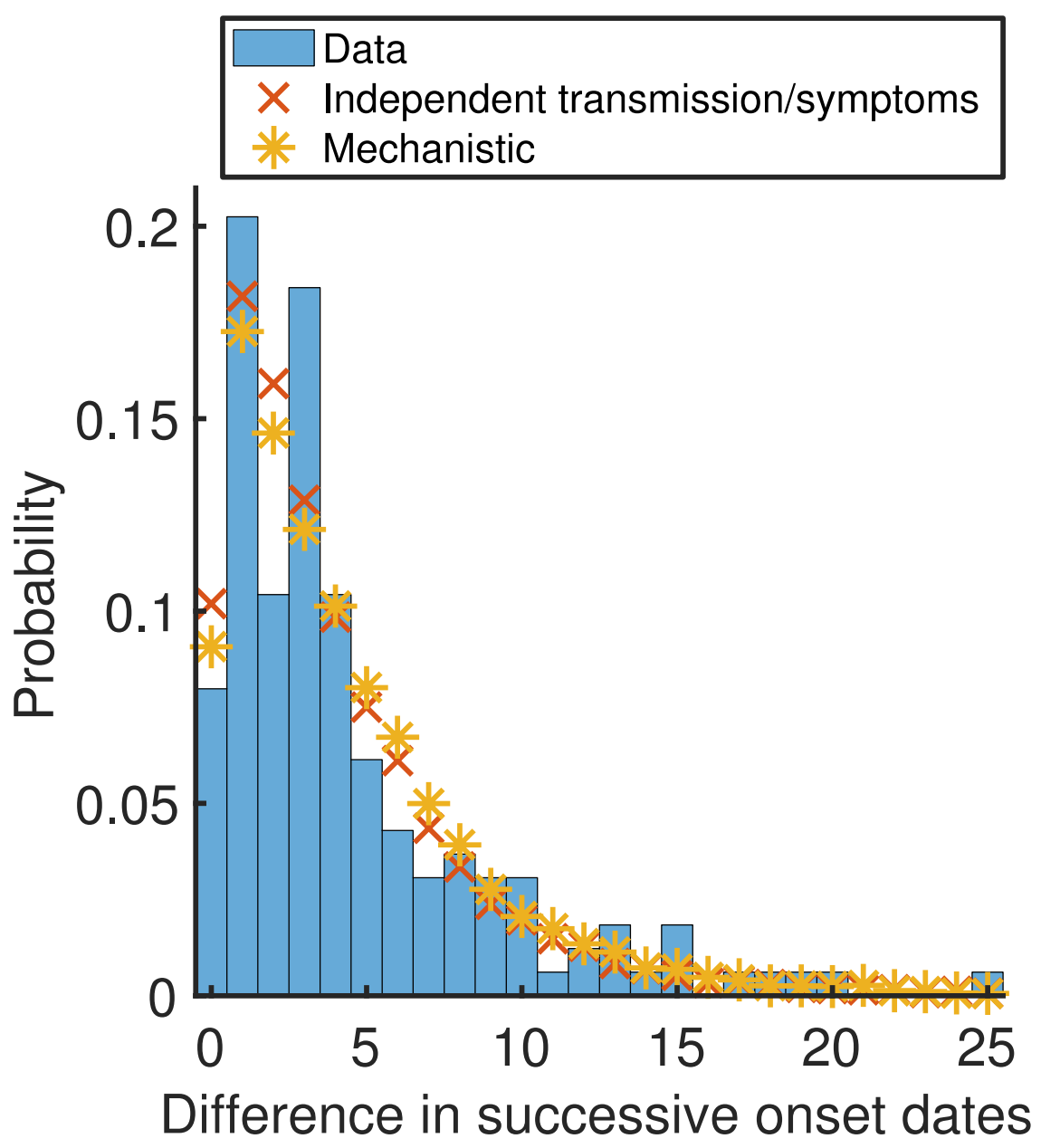

Figure 1-figure supplement 3. Observed and model-predicted distributions of intervals between

successive symptom onset dates. Using posterior mean parameter estimates, we predicted the distribution of

1081 the difference in successive symptom onset dates (this differs from the serial interval because an infected individual may not have been infected by the previous household member to develop symptoms) under the fitted independent transmission and symptoms model (red crosses) and mechanistic model (yellow stars). These distributions were compared to the UK household data (blue bars). The distributions for the fitted models were obtained by generating synthetic data from 16,700 households using the same distribution of household sizes as in the UK data. 
A.

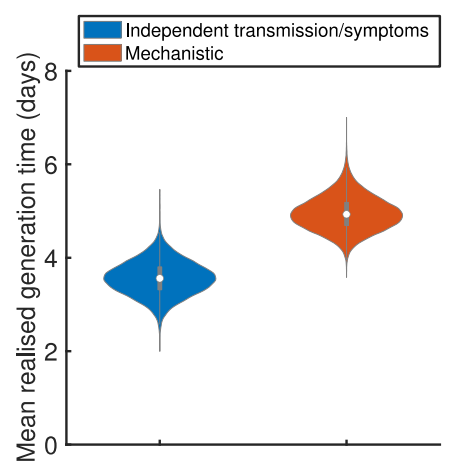

B.

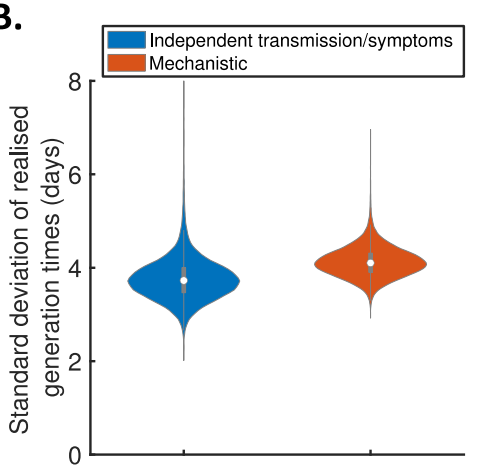

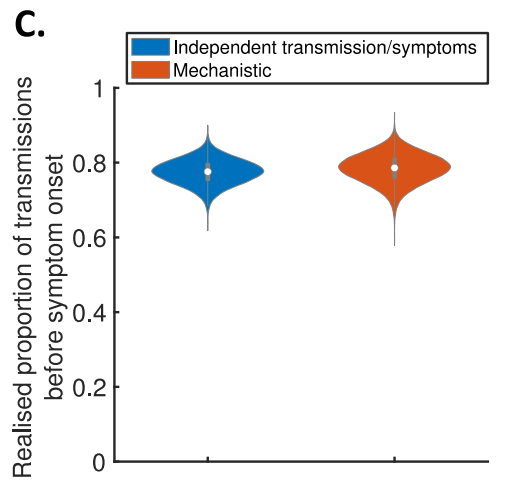

Figure 1-figure supplement 4. Generation times within study households. Violin plots indicating posterior distributions of the mean (A) and standard deviation (B) of realised generation times in the study households, and the realised proportion of transmissions occurring prior to symptom onset (C), for the independent transmission and symptoms model (blue) and mechanistic model (red). 
A.

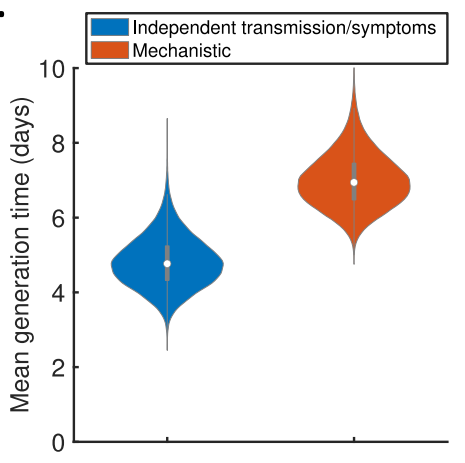

B.

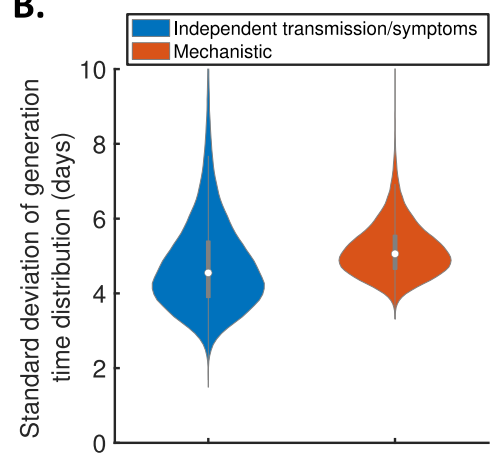

C.

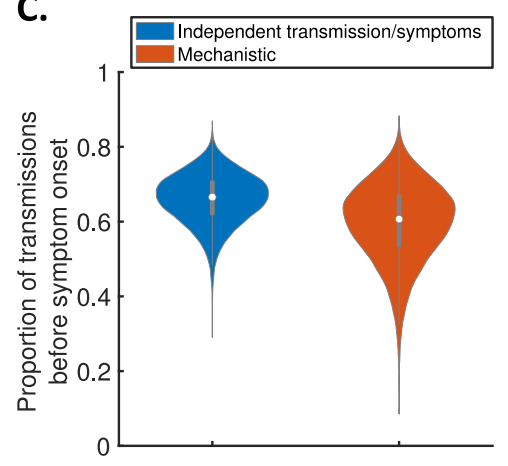

D.

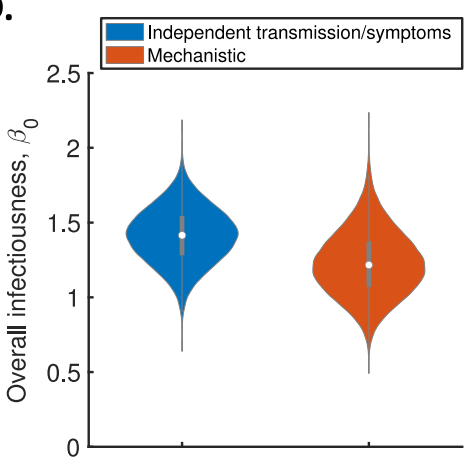

E.

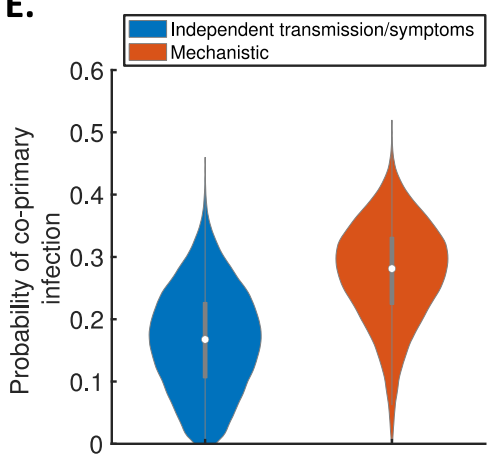

Figure 1-figure supplement 5. Estimates of the generation time accounting for the possibility of co-

primary cases. Violin plots indicating posterior distributions of the mean (A) and standard deviation (B) of the generation time distribution, proportion of transmissions occurring prior to symptom onset (among infectors who develop symptoms; $\mathrm{C}$ ), overall infectiousness parameter, $\beta_{0}(\mathrm{D})$, and probability of co-primary infection (E), for the independent transmission and symptoms model (blue) and mechanistic model (red), when the possibility of co-primary cases was included in our approach (see the Appendix). 
A.

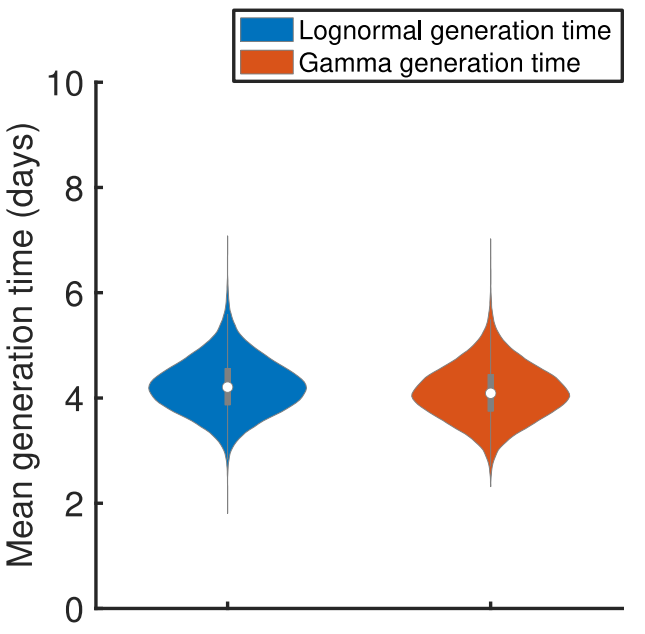

C.

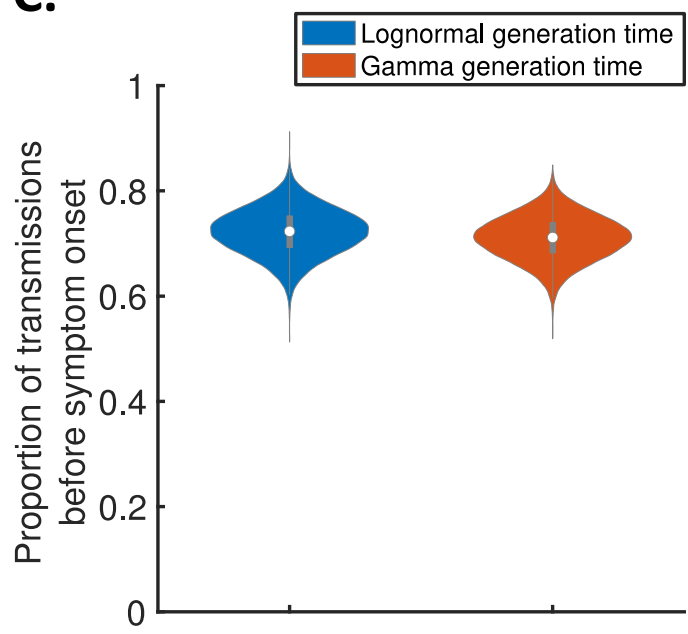

B.

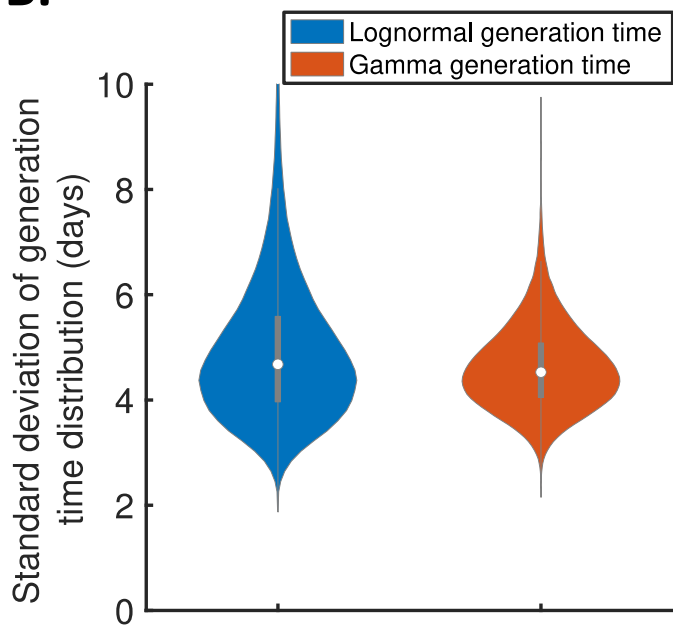

D.

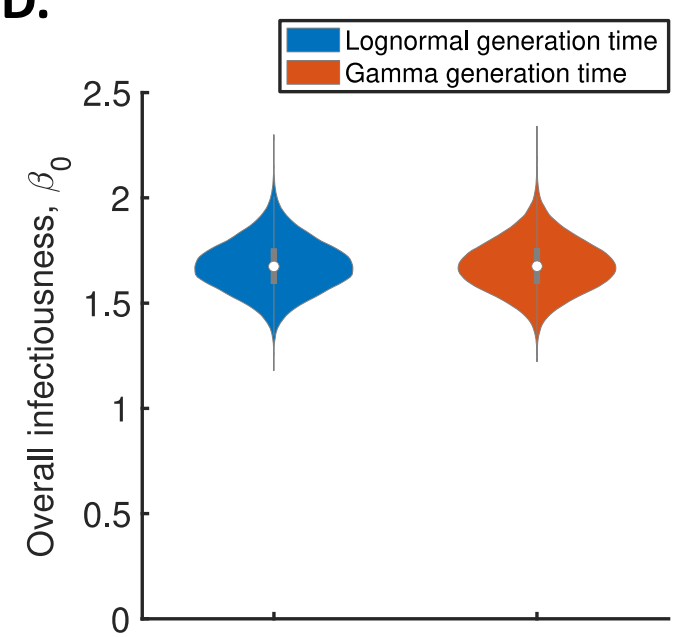

1103 Figure 1-figure supplement 6. Sensitivity of the results to the functional form of the generation time

1104 distribution for the independent transmission and symptoms model. Violin plots indicating posterior

1105 distributions of the mean (A) and standard deviation (B) of the generation time distribution, proportion of

1106 transmissions occurring prior to symptom onset (among infectors who develop symptoms; C), and overall

1107 infectiousness parameter, $\beta_{0}(\mathrm{D})$, for the independent transmission and symptoms model, when the generation

1108 time was assumed to follow either a lognormal (blue, as in the main text) or a gamma (red) distribution, and the

1109 incubation period distribution followed a lognormal distribution (as in the main text). 
A.

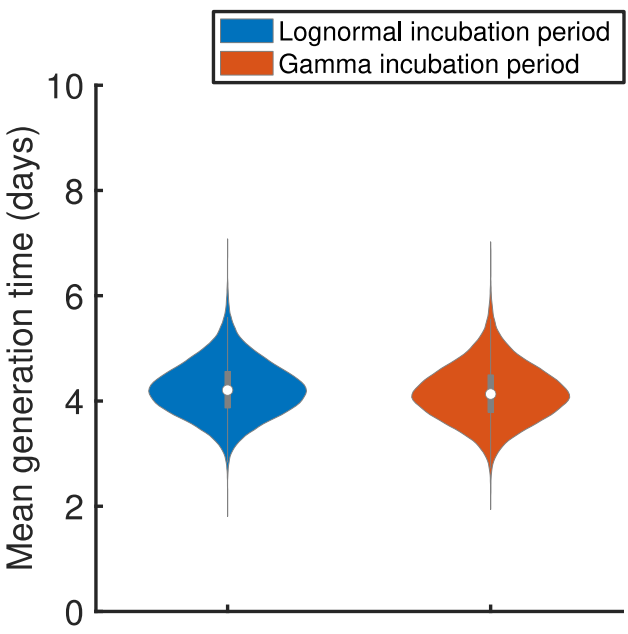

C.

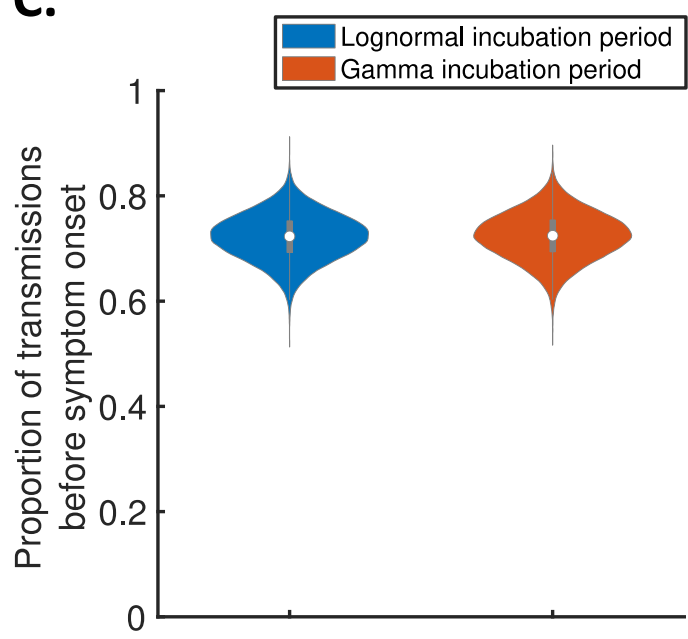

B.

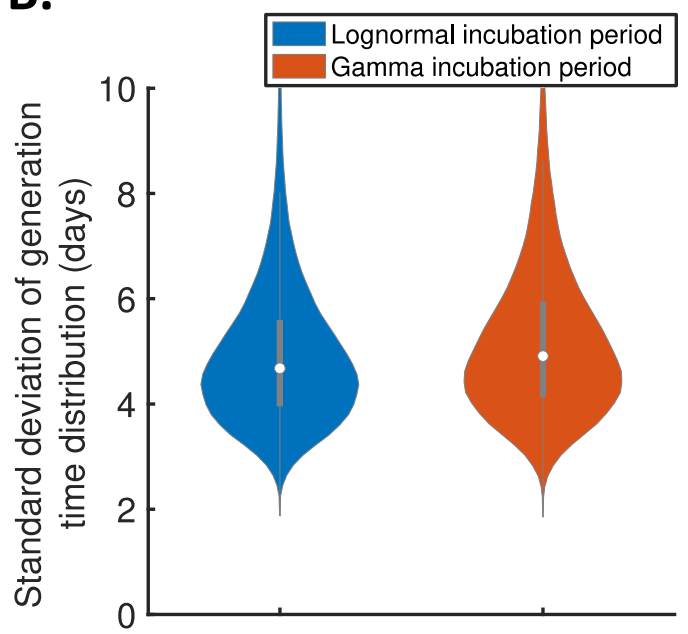

D.

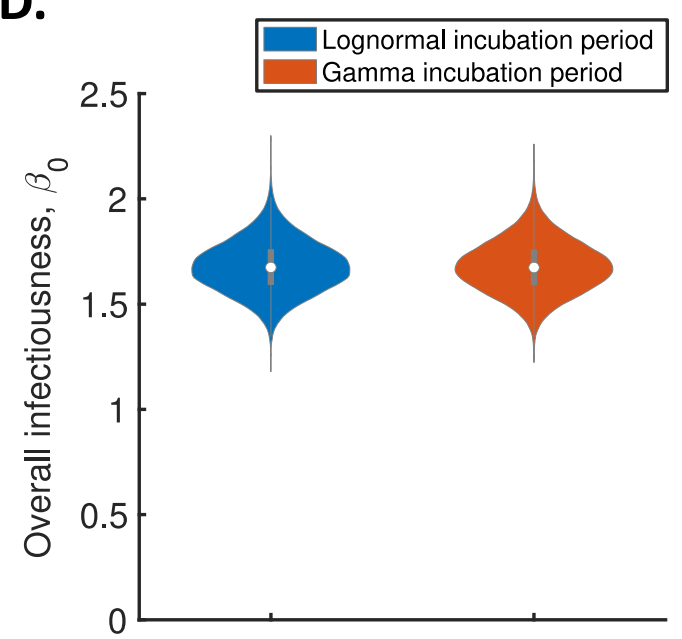

1112 Figure 1-figure supplement 7. Sensitivity of the results to the functional form of the incubation period

1113 distribution for the independent transmission and symptoms model. Violin plots indicating posterior

1114 distributions of the mean (A) and standard deviation (B) of the generation time distribution, proportion of

1115 transmissions occurring prior to symptom onset (among infectors who develop symptoms; C), and overall

1116 infectiousness parameter, $\beta_{0}(\mathrm{D})$, for the independent transmission and symptoms model, when the incubation

1117 period was assumed to follow either a lognormal distribution (blue, as in the main text) or a gamma distribution

1118 with the same mean and standard deviation (red), and the generation time followed a lognormal distribution (as

1119 in the main text). 
A.

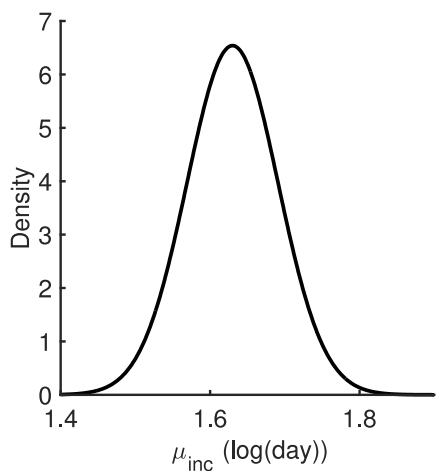

D.

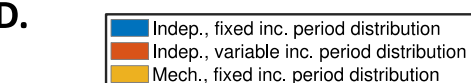
Mech., fixed inc. period distribution

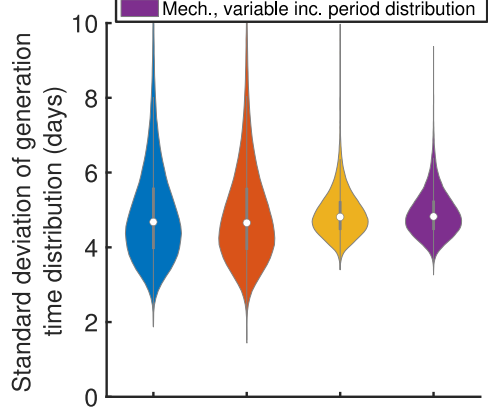

B.

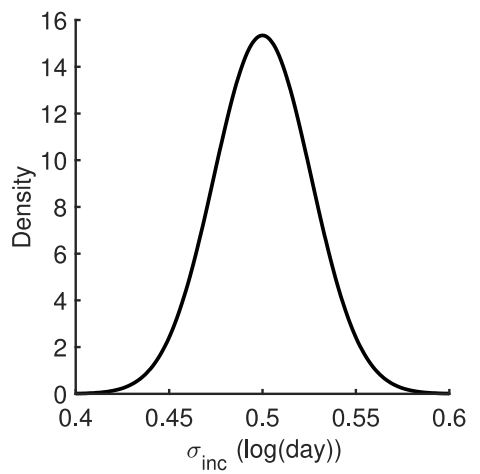

E.

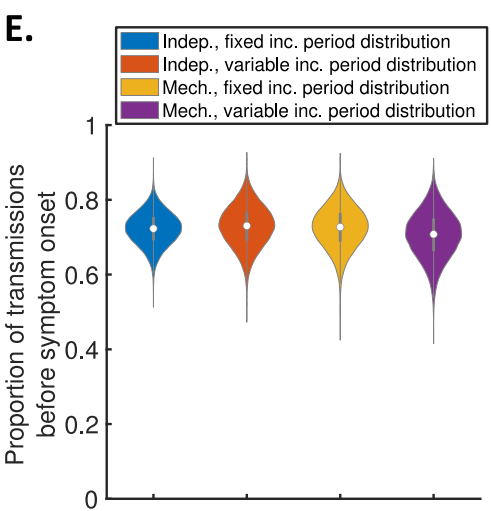

C.

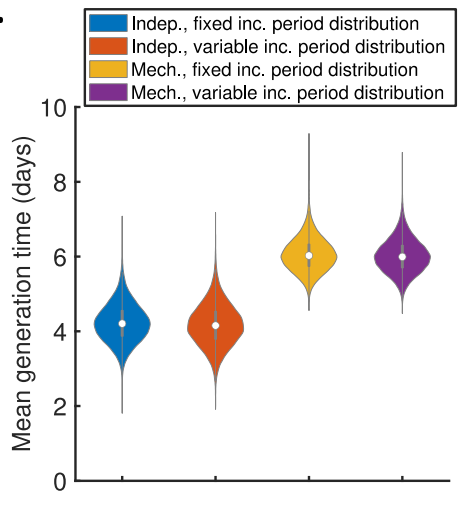

F.

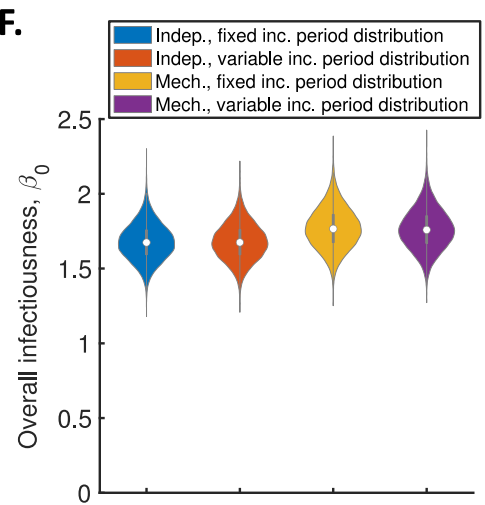

Figure 1-figure supplement 8. Sensitivity of the results to the incubation period distribution. A-B.

1123 Uncertainty in the incubation period distribution was accounted for by updating the parameters, $\mu_{i n c}$ and $\sigma_{i n c}$, of

1124 a lognormal incubation period distribution (these parameters represent the mean and standard deviation of the natural logarithm of the incubation period distribution, respectively) alongside unknown model parameters during the MCMC procedure. Independent normal prior distributions (truncated at zero) consistent with the 95\% confidence intervals obtained in (McAloon et al., 2020) were assumed for $\mu_{i n c}$ (prior mean $1.63 \log$ (day), standard deviation 0.061, 95\% CrI 1.51-1.75; panel A) and $\sigma_{\text {inc }}$ (prior mean $0.5 \log$ (day), standard deviation $0.026,95 \%$ CrI $0.45-0.55$; panel B). This incubation period was used directly when evaluating the likelihood in the independent transmission and symptoms model. In the mechanistic model, we assumed a gamma distributed incubation period with the same mean and standard deviation as a lognormal distribution with parameters $\mu_{i n c}$ and $\sigma_{i n c}$. C-F. Violin plots indicating posterior distributions of the mean (C) and standard deviation (D) of the generation time distribution, proportion of transmissions occurring prior to symptom onset (among infectors who develop symptoms; E), and overall infectiousness parameter, $\beta_{0}(\mathrm{~F})$, for the independent transmission and symptoms model with either a fixed (as in the main text; blue) or variable (i.e., accounting for uncertainty in $\mu_{i n c}$ and $\sigma_{i n c}$ as described above; red) incubation period distribution, and for the mechanistic model with a fixed (orange) or variable (purple) incubation period distribution. 
A.
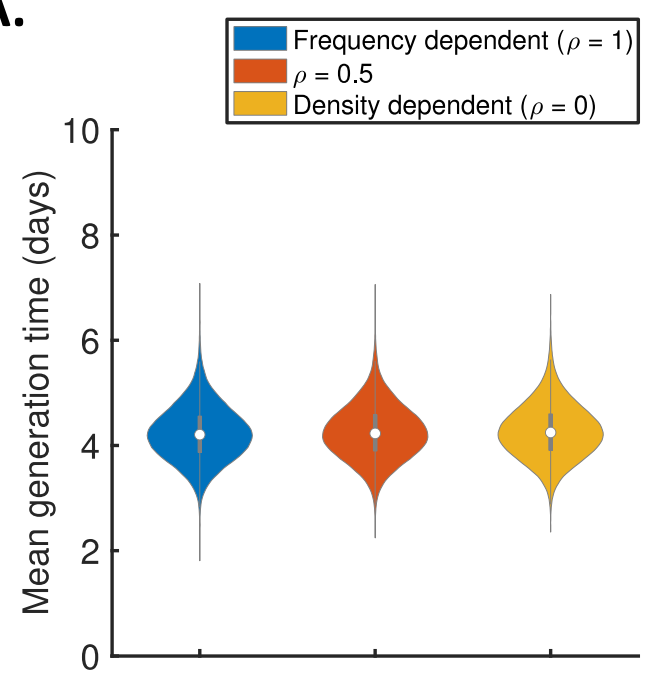

c.
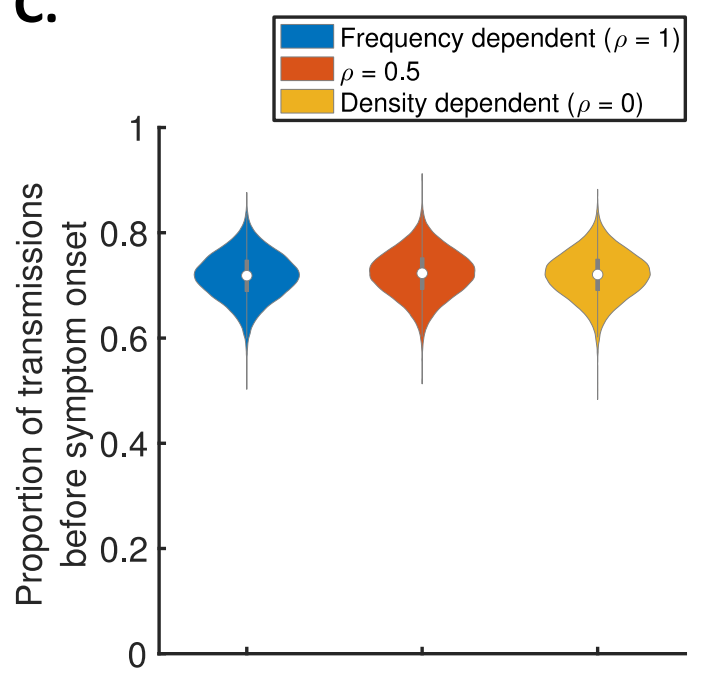
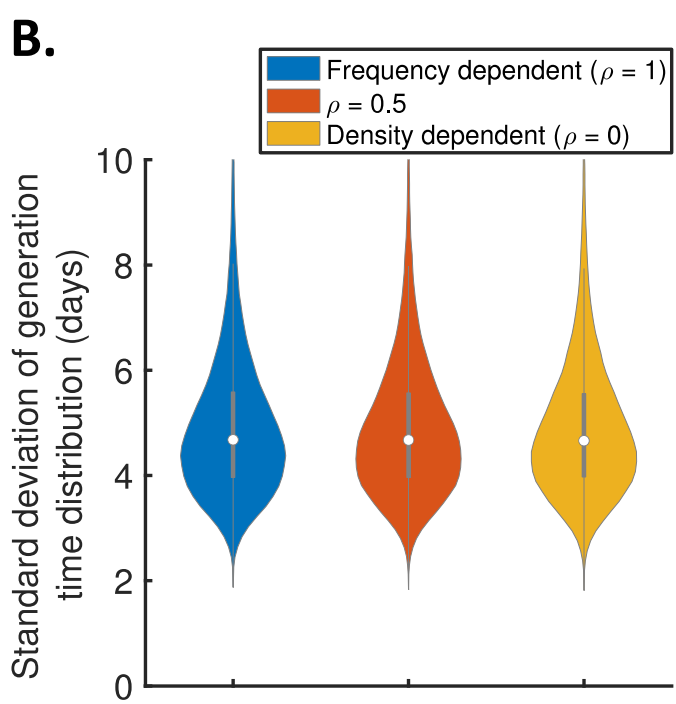

D.

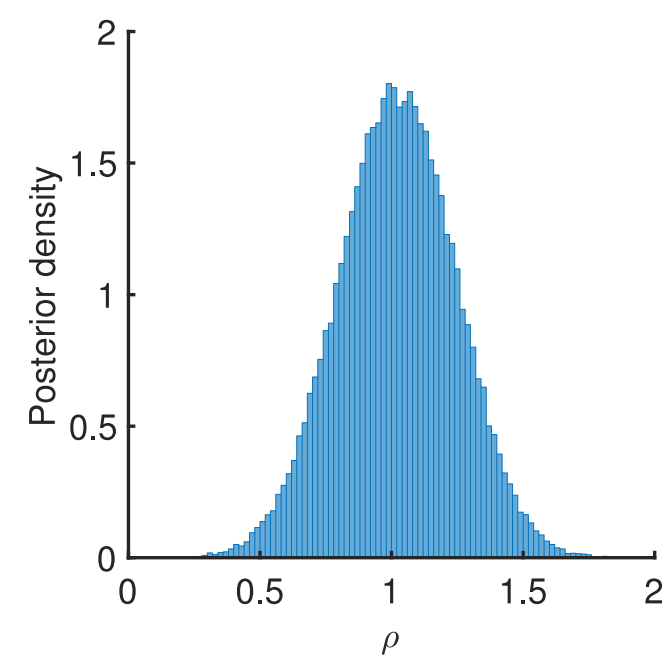

1139 Figure 1-figure supplement 9. Sensitivity of the results to the dependency of transmission on the

1140 household size. A-C. Violin plots indicating the posterior distributions of the mean (A) and standard deviation

1141 (B) of the generation time distribution, and proportion of transmissions occurring prior to symptom onset

1142 (among infectors who develop symptoms; C), for the independent transmission and symptoms model under

1143 different assumptions about the dependency of transmission on the household size. In these panels,

1144 infectiousness is assumed to scale with $n^{-\rho}$, where $n$ is the household size, for $\rho=1$ (frequency-dependent

1145 transmission, blue), $\rho=0.5$ (red), $\rho=0$ (density-dependent transmission, orange). D. Posterior distribution of

1146 the dependency, $\rho$, when it was fitted to the data (alongside other model parameters), assuming a uniform prior 1147 for $\rho$ 
A.

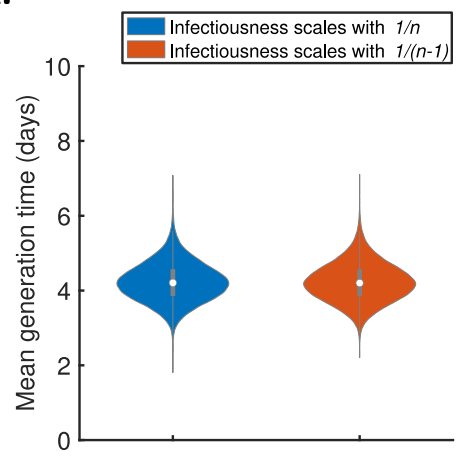

B.

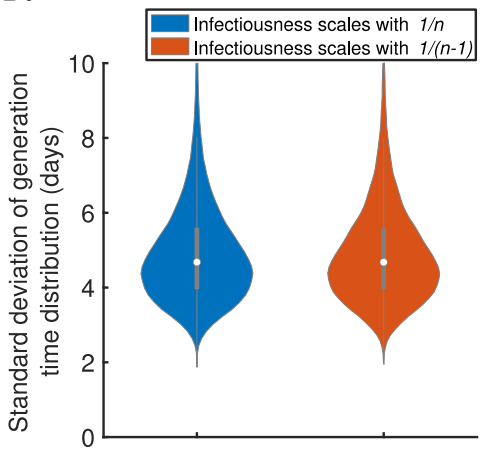

C.

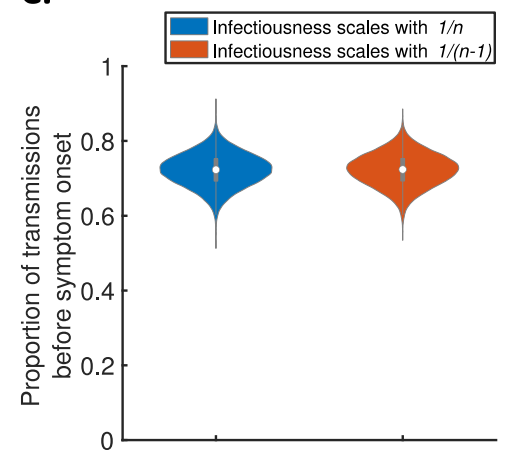

1150 Figure 1-figure supplement 10. Further sensitivity of the results to the dependency of transmission on the

1151 household size. Violin plots indicating the posterior distributions of the mean (A) and standard deviation (B) of

1152 the generation time distribution, and proportion of transmissions occurring prior to symptom onset (among

1153 infectors who develop symptoms; C), for the independent transmission and symptoms model under different

1154 assumptions about the dependency of transmission on the household size. In these panels, infectiousness is

1155 assumed to scale with either $1 / n$ (blue) or $1 /(n-1)$ (red). 
A.

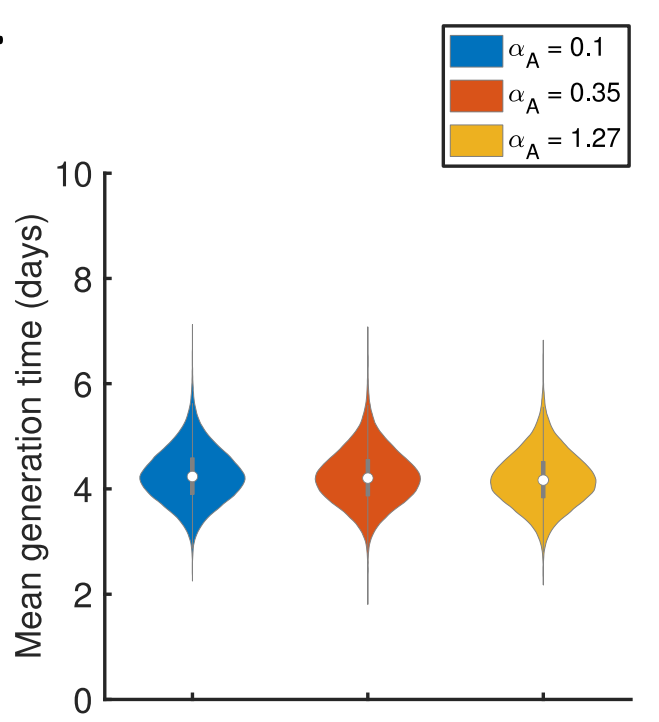

C.
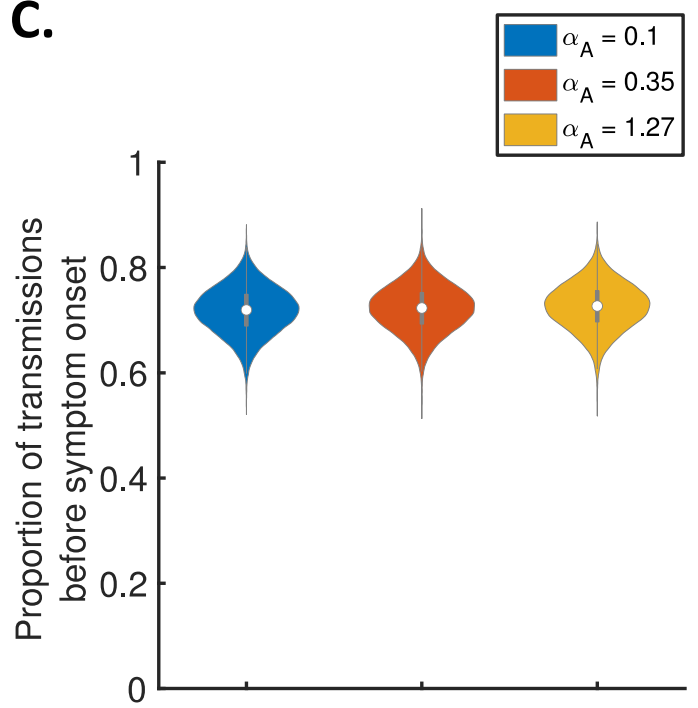

B.
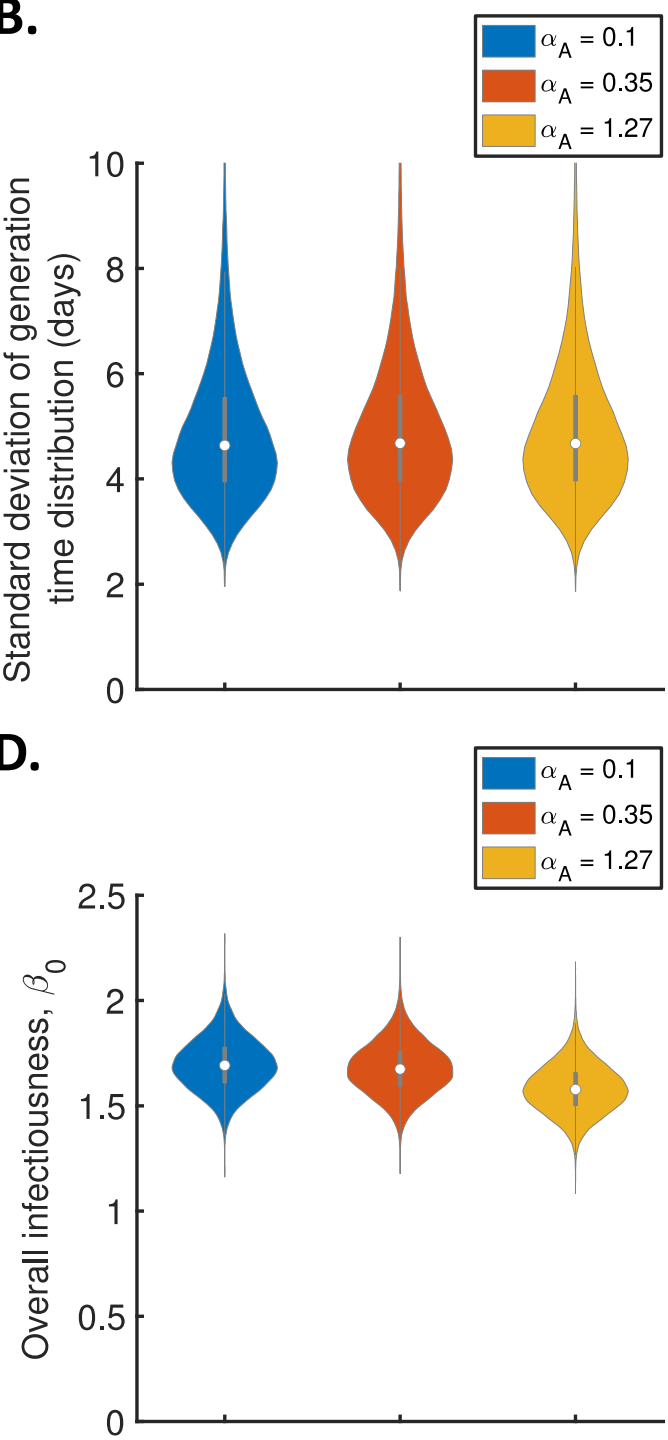

1158 Figure 1-figure supplement 11. Sensitivity of the results to the relative infectiousness of entirely

1159 asymptomatic infected hosts. Violin plots indicating posterior distributions of the mean (A) and standard

1160 deviation (B) of the generation time distribution, proportion of transmissions occurring prior to symptom onset

1161 (among infectors who develop symptoms; C), and overall infectiousness parameter, $\beta_{0}(\mathrm{D})$, for the independent transmission and symptoms model, when the relative infectiousness of asymptomatic hosts (compared to hosts who develop symptoms, at the same time since infection) was assumed to be $\alpha_{A}=0.1$ (blue), $\alpha_{A}=0.35$ (red) and $\alpha_{A}=1.27$ (orange). 
A.

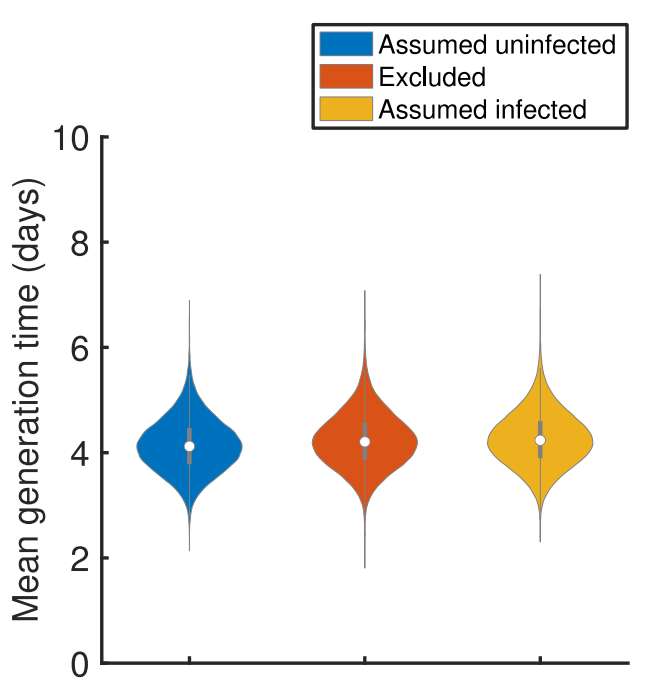

C.

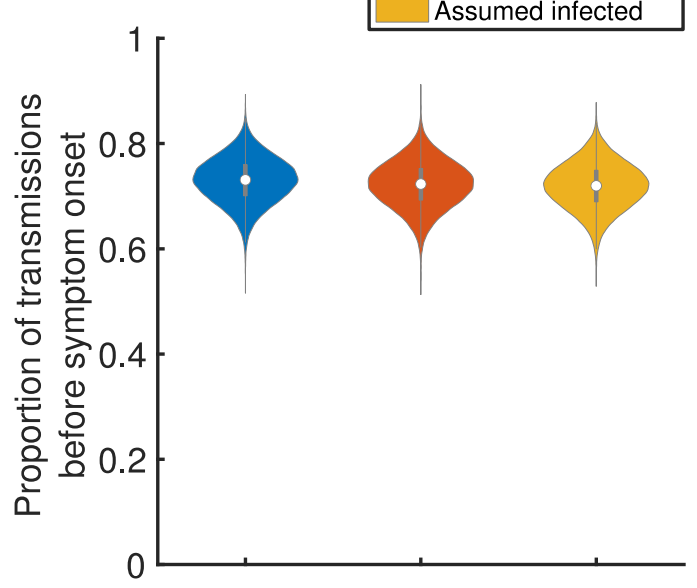

B.
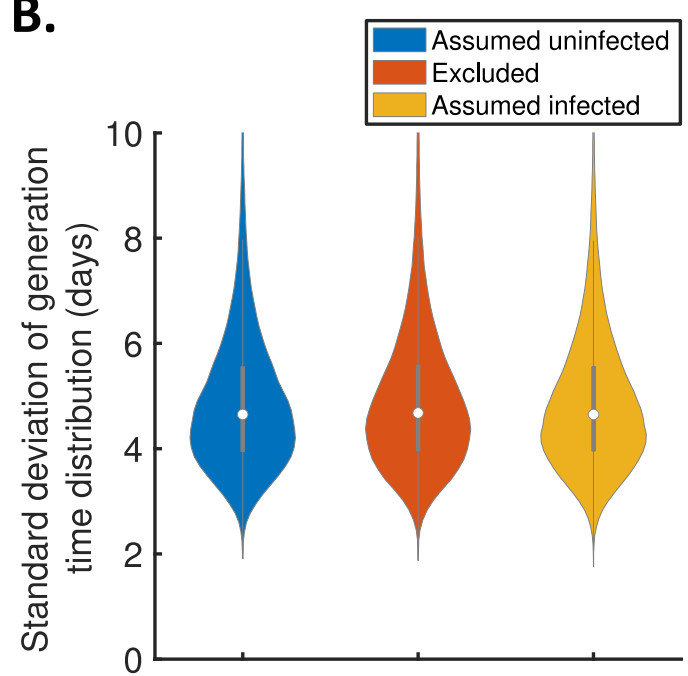

D.
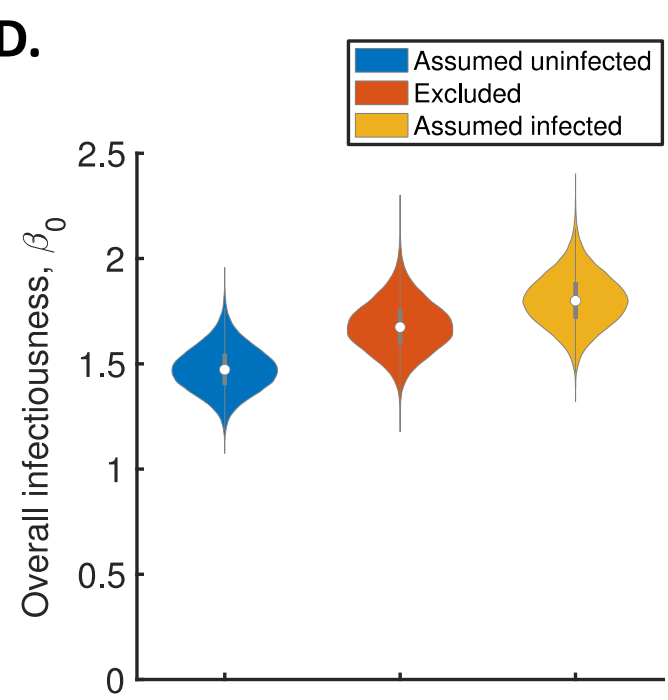

1167 Figure 1-figure supplement 12. Sensitivity of the results to the exclusion of household members of

1168 unknown infection status. A-C. Violin plots indicating the posterior distributions of the mean (A) and standard

1169 deviation (B) of the generation time distribution, proportion of transmissions occurring prior to symptom onset

1170 (among infectors who develop symptoms; C), and overall infectiousness parameter, $\beta_{0}$ (D), for the independent

1171 transmission and symptoms model, when individuals of unknown infection status were assumed all uninfected

1172 (blue), excluded (red), or assumed all infected (orange). 
A.

1174

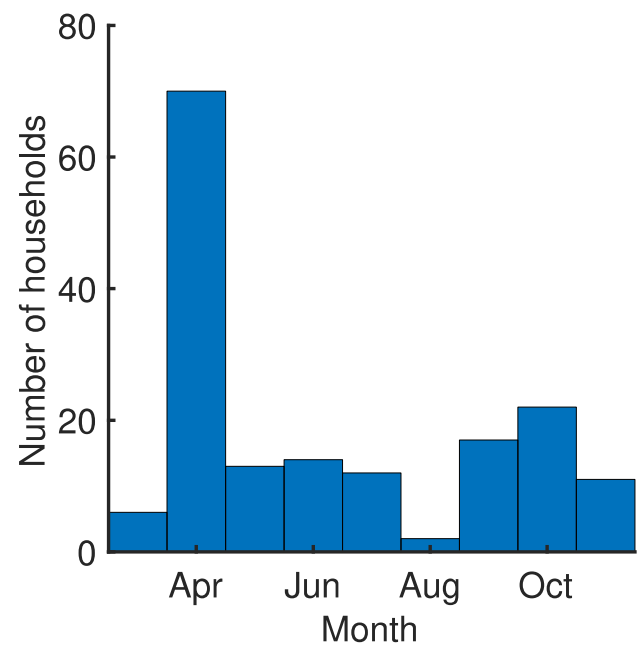

B.

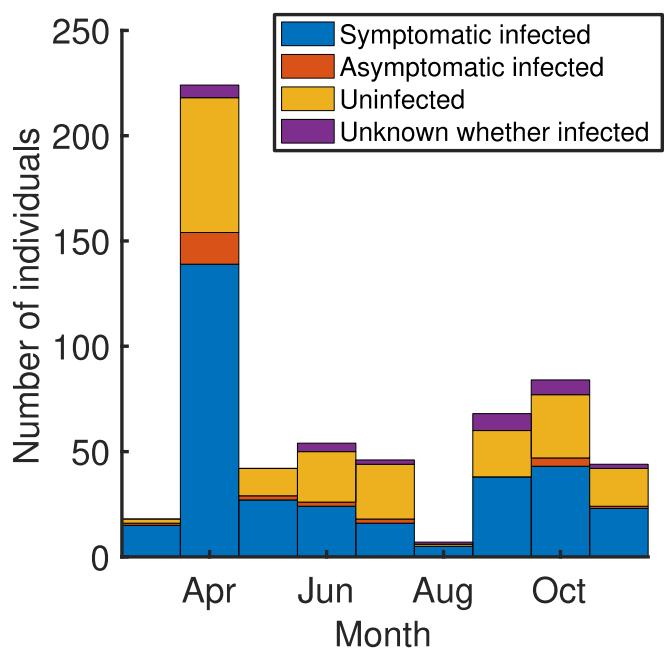

1175 Figure 3-figure supplement 1. Number of study households and household members by recruitment

1176 month. A. Bars indicating the number of households recruited each month into the study from which we

1177 obtained the household transmission data used in our analyses, from March to November 2020 (excluding five

1178 households that were excluded from our analyses as described in Materials and Methods). B. Stacked bars

1179 indicating the total number of individuals within the households recruited each month, distinguishing between

1180 those who were infected and developed symptoms (blue), those who were infected but never developed

1181 symptoms (red), those who remained uninfected (yellow), and those for whom it was unknown whether or not 1182 they were infected (purple). 
A.

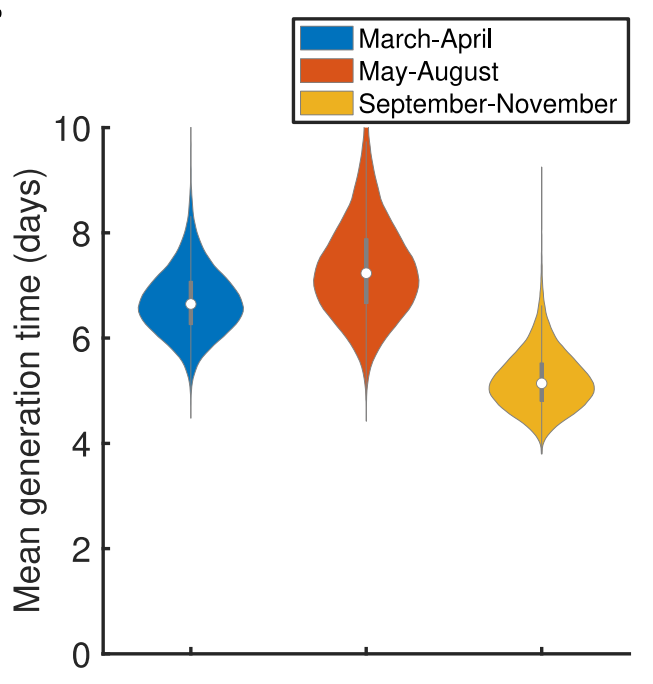

C.

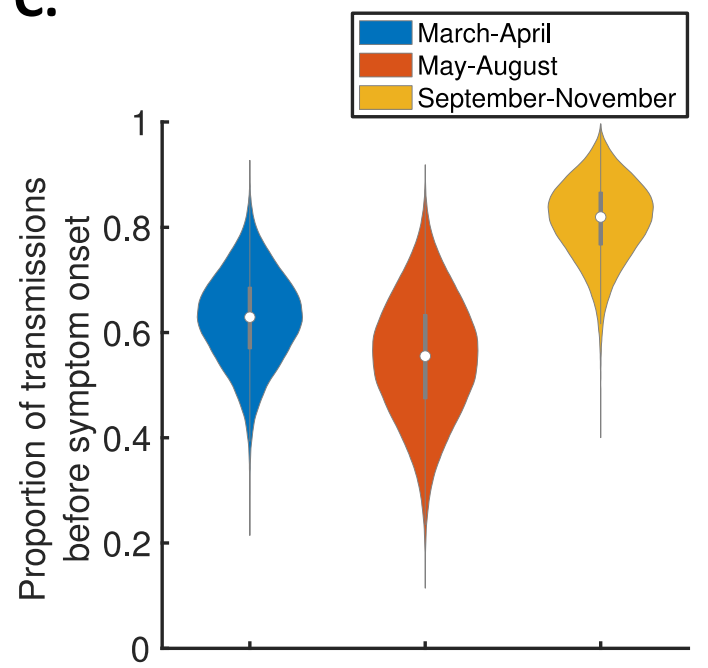

B.

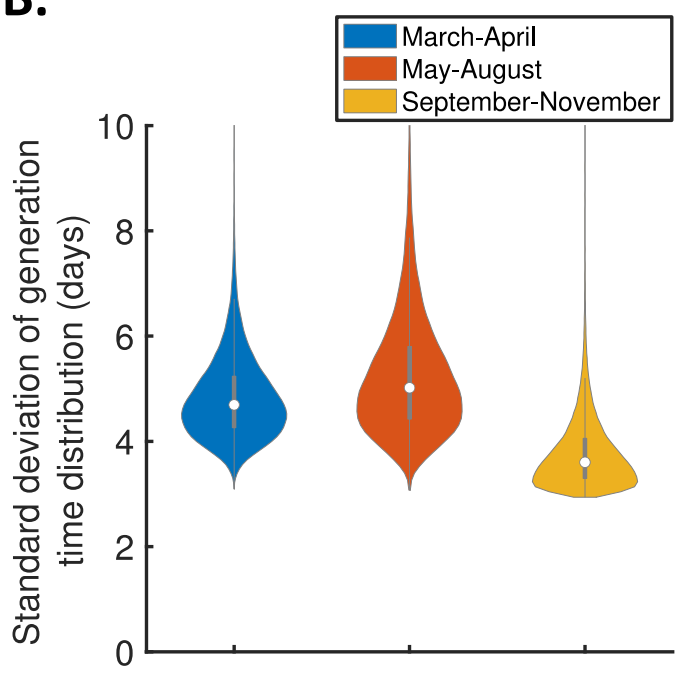

D.
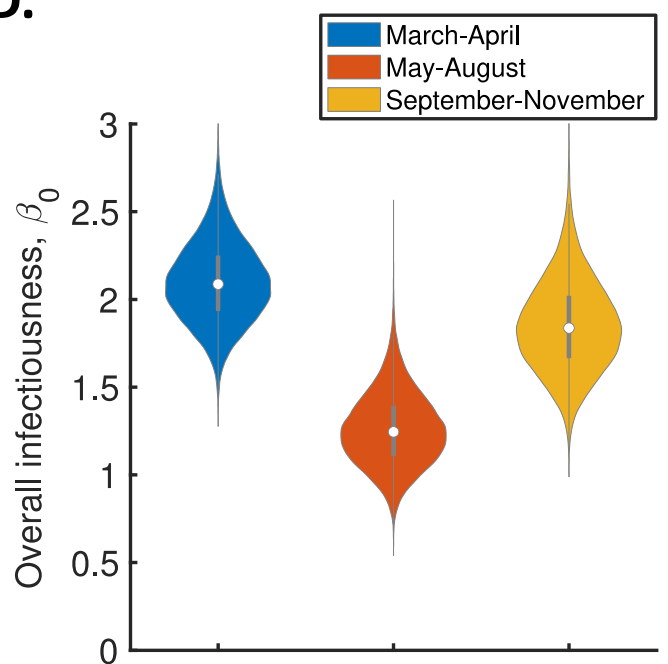

1185 Figure 3-figure supplement 2. Temporal changes in the generation time for the mechanistic model. Violin

1186 plots indicating posterior distributions of the mean (A) and standard deviation (B) of the generation time

1187 distribution, proportion of transmissions occurring prior to symptom onset (among infectors who develop symptoms; C), and overall infectiousness parameter, $\beta_{0}(\mathrm{D})$, for the mechanistic model fitted to data from 1189 March-April (blue), May-August (red) or September-November 2020 (orange). 

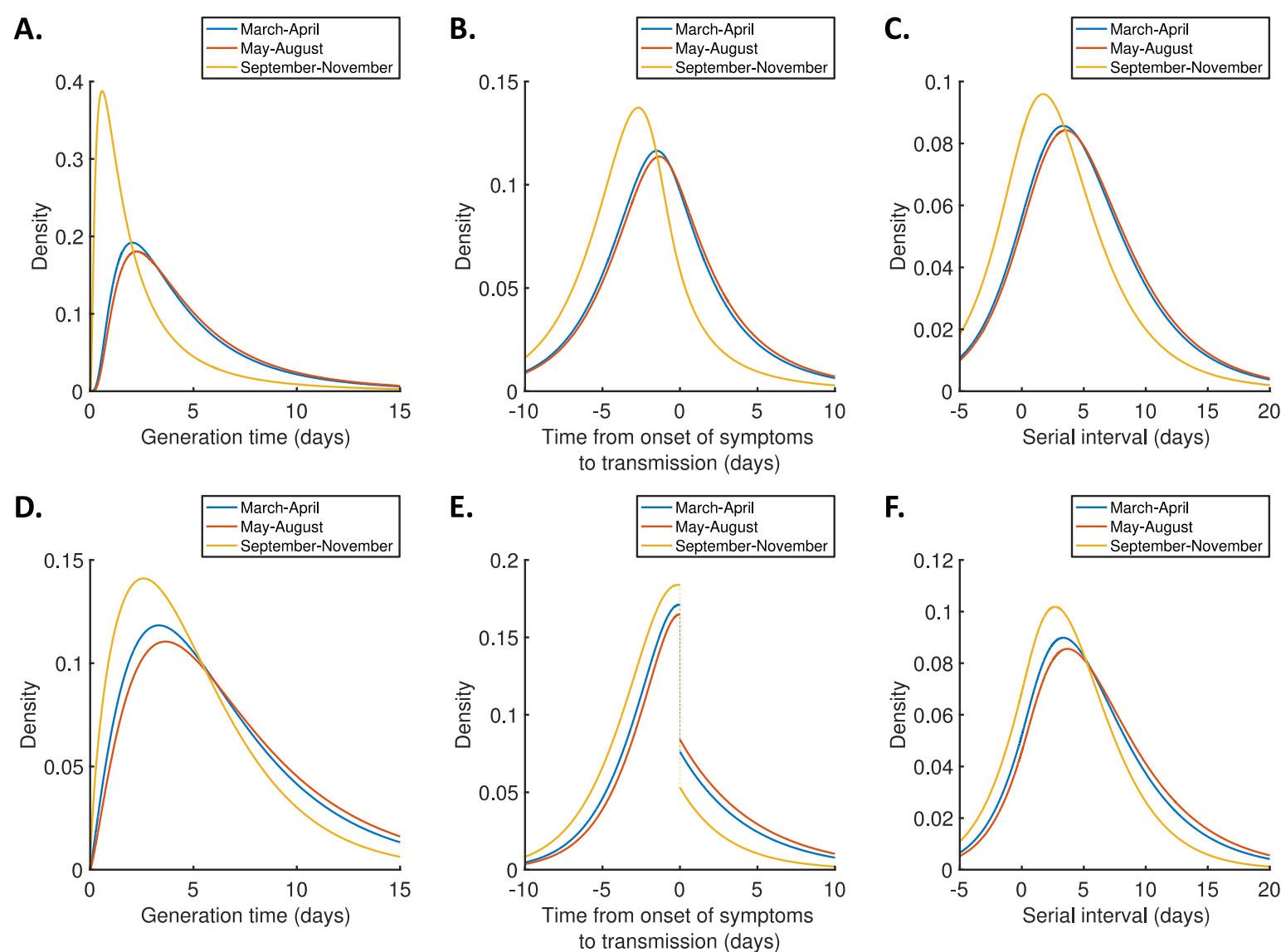

1192 Figure 3-figure supplement 3. Temporal changes in the generation time, TOST and serial interval

1193 distributions. A-C. Inferred generation time (A), TOST (B) and serial interval (C) distributions, obtained using

1194 point estimate (posterior mean) parameters for the independent transmission and symptoms model fitted to data 1195 from March-April (blue), May-August (red) or September-November 2020 (orange). D-F. Equivalent panels to 1196 A-C for the mechanistic model. 
A.

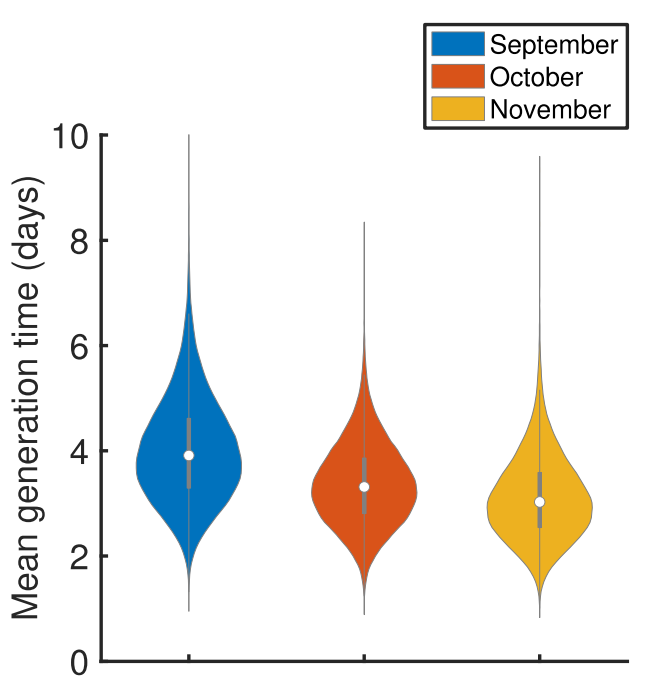

C.

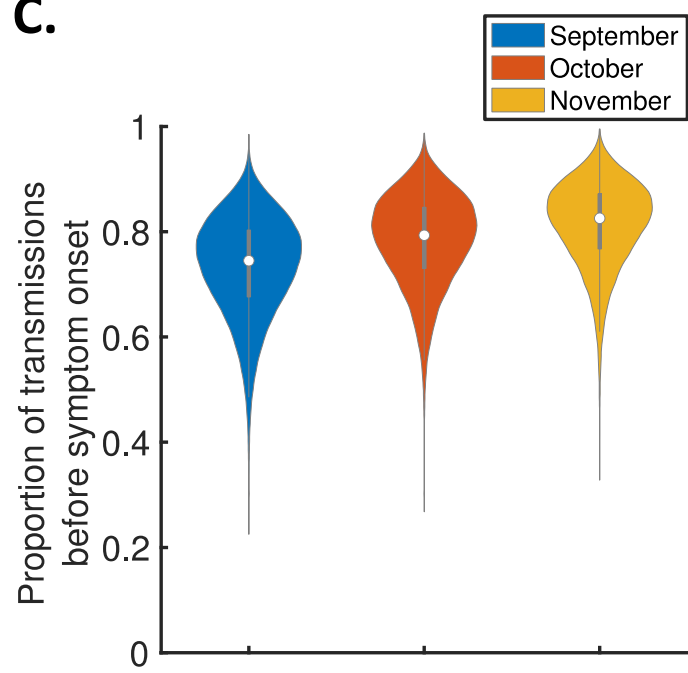

B.
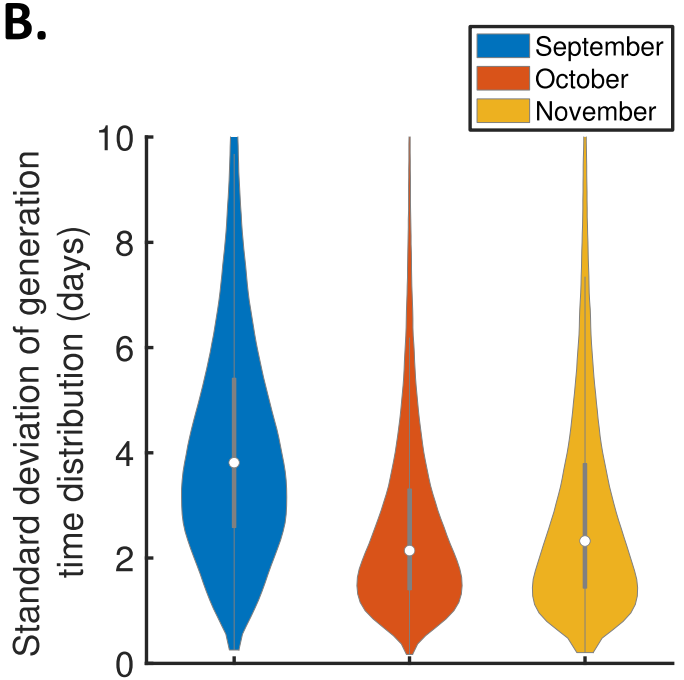

D.

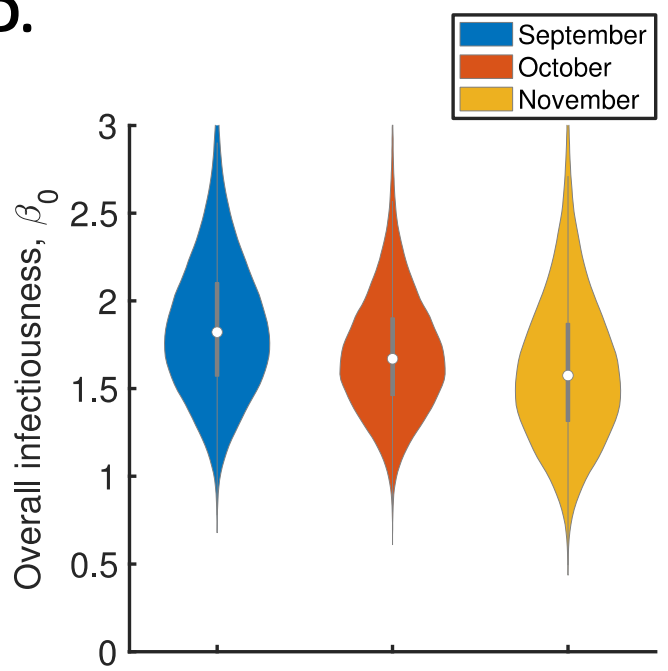

1198 Figure 3-figure supplement 4. Monthly changes in the generation time from September-November 2020.

1199 Violin plots indicating posterior distributions of the mean (A) and standard deviation (B) of the generation time 1200 distribution, proportion of transmissions occurring prior to symptom onset (among infectors who develop symptoms; C), and overall infectiousness parameter, $\beta_{0}(\mathrm{D})$, for the independent transmission and symptoms model fitted to data from September (blue), October (red) or November 2020 (orange). 
A.

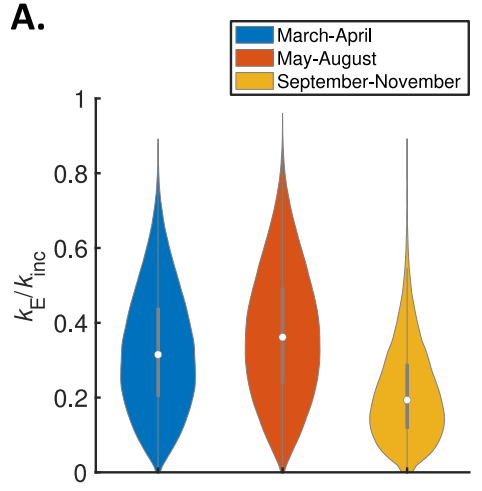

B.

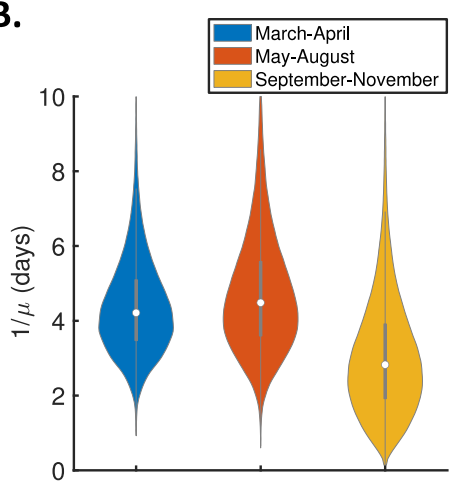

C.

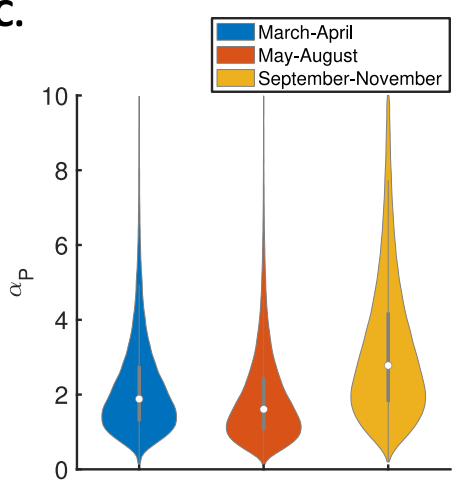

Figure 3-figure supplement 5. Temporal changes in fitted parameters for the mechanistic model. Violin

1206 plots indicating posterior distributions of estimated model parameters for the mechanistic model fitted to data

1207 from March-April (blue), May-August (red) or September-November 2020 (orange). A. Ratio of mean durations

1208 of the latent $(E)$ and incubation (combined $E$ and $P$ ) periods, $k_{E} / k_{i n c}$. B. Mean symptomatic infectious $(I)$

1209 period, $1 / \mu$. C. Ratio of transmission rates in the presymptomatic infectious $(P)$ and symptomatic infectious $(I)$

1210 stages of infection, $\alpha_{P}$. Corresponding estimates of the overall infectiousness, $\beta_{0}$ (the other fitted model

1211 parameter) are shown in Figure 3-figure supplement 2D.

1212 
A.

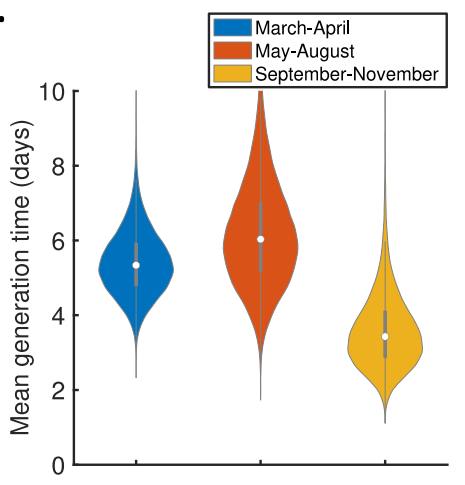

D.

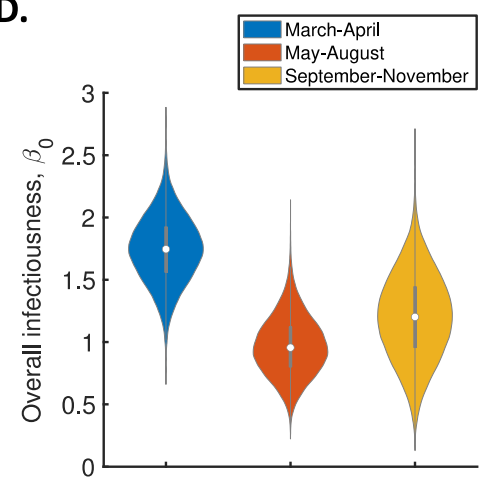

B.

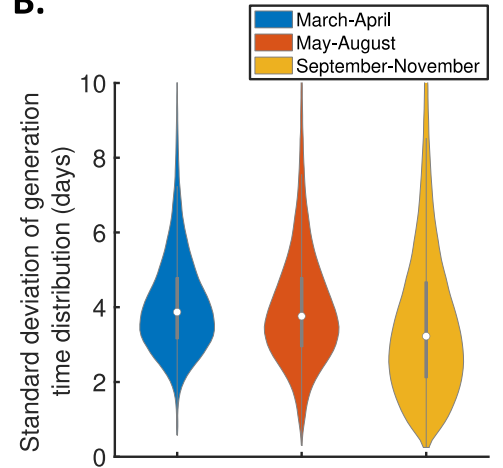

E.

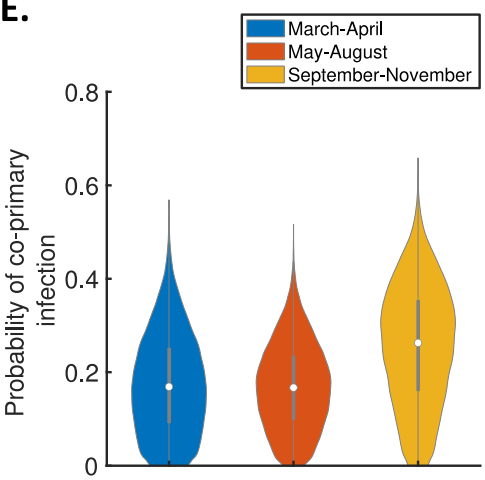

C.

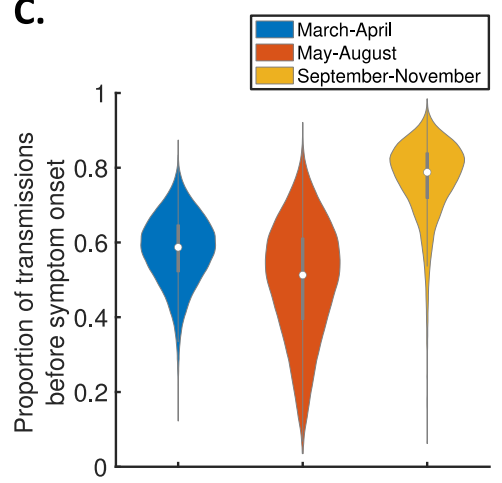

1214 Figure 3-figure supplement 6. Temporal changes in the generation time accounting for the possibility of co-primary cases. Violin plots indicating posterior distributions of the mean (A) and standard deviation (B) of

1216 the generation time distribution, proportion of transmissions occurring prior to symptom onset (among infectors

1217 who develop symptoms; C), overall infectiousness parameter, (D), and probability of co-primary infection (E),

1218 for the independent transmission and symptoms model fitted to data from March-April (blue), May-August (red) or September-November 2020 (orange), when the possibility of co-primary cases was included in our approach (see the Appendix). 


\section{Appendix:}

1222

1223

1224

1225

1226

1227

1228

1229

1230

1231

1232

1233

1234

1235

1236

1237

1238

1239

1240

\section{Details of mechanistic model}

In this model, each infected host (who develops symptoms) progresses through independent latent $(E)$, presymptomatic infectious $(P)$ and symptomatic infectious $(I)$ stages of infection. The infectiousness of the host during the $P$ and $I$ stages is denoted by $\beta_{P}$ and $\beta_{I}$, respectively, and we denote the ratio $\alpha_{P}=\beta_{P} / \beta_{I}$. We assumed the duration of each stage, denoted $y_{E / P / I}$, to be gamma distributed:

$$
\begin{aligned}
y_{E} & \sim \operatorname{Gamma}\left(k_{E}, 1 /\left(k_{\text {inc }} \gamma\right)\right), \\
y_{P} & \sim \operatorname{Gamma}\left(k_{P}, 1 /\left(k_{\text {inc }} \gamma\right)\right), \\
y_{I} & \sim \operatorname{Gamma}\left(k_{I}, 1 /\left(k_{I} \mu\right)\right),
\end{aligned}
$$

where we write $X \sim \operatorname{Gamma}(a, b)$ for a gamma distributed random variable with shape parameter $a$ and scale parameter $b$. We assumed that $k_{E}+k_{P}=k_{\text {inc }}$, so that the incubation period, $\tau_{i n c}=y_{E}+y_{P}$, is gamma distributed, with

$$
\tau_{\text {inc }} \sim \operatorname{Gamma}\left(k_{\text {inc }}, 1 /\left(k_{\text {inc }} \gamma\right)\right) .
$$

We fixed the values of the parameters $k_{i n c}$ and $1 / \gamma$ (which represent the shape parameter of the incubation period distribution and the reciprocal of the mean incubation period, respectively) in order to obtain the specified incubation period distribution (the exact values that we assumed are given in Appendix 1-table 1). For simplicity, we also assumed that $k_{I}=1$, so the symptomatic infectious period is exponentially distributed. The parameters $k_{E}$ (the shape parameter of the latent $(E)$ period distribution), $1 / \mu$ (the mean symptomatic infectious ( $I$ ) period) and $\alpha_{P}$ (the ratio between the transmission rates of hosts in the $P$ and $I$ stages) were estimated when we fitted the model to the household transmission data. 
Hosts who remain asymptomatic throughout infection were assumed to follow the same $E / P / I$

1242

1243

1244

1245

1246

1247

1248

1249

1250

1251

1252

1253

1254

1255

1256

1257

stages, although in this case the distinction between the $P$ and $I$ stages has no epidemiological meaning. Stage durations, as well as the value of $\alpha_{P}$, were assumed to be identical for entirely asymptomatic hosts and those who develop symptoms, so that the generation time distribution is the same for all infected hosts.

\section{Conditional infectiousness}

For a host who develops symptoms, conditional on incubation period $\tau_{\text {inc }}$, the expected infectiousness at time since infection $\tau$ is (Hart et al., 2021)

$$
\beta\left(\tau \mid \tau_{\text {inc }}\right)=\left\{\begin{array}{cc}
\alpha_{P} C\left(\beta_{0} / n\right)\left(1-F_{B e t a}\left(1-\tau / \tau_{\text {inc }} ; k_{P}, k_{E}\right)\right), & 0<\tau<\tau_{\text {inc }} \\
C\left(\beta_{0} / n\right)\left(1-F_{I}\left(\tau-\tau_{\text {inc }}\right)\right), & \tau>\tau_{\text {inc }} .
\end{array}\right.
$$

Here, $\beta_{0}$ is the overall infectiousness parameter (see Materials and Methods in the main text), $n$ is the household size, $F_{I}(y)$ is the cumulative distribution of the duration of the $I$ stage, $F_{\text {Beta }}(x ; a, b)$ is the cumulative distribution of a beta distributed random variable with shape parameters $a$ and $b$, and

$$
C=\frac{k_{\text {inc }} \gamma \mu}{\alpha_{P} k_{P} \mu+k_{\text {inc }} \gamma}
$$

The cumulative conditional infectiousness can therefore be calculated to be

$$
\begin{aligned}
& B\left(\tau \mid \tau_{i n c}\right)=\int_{0}^{\tau} \beta\left(\tilde{\tau} \mid \tau_{i n c}\right) \mathrm{d} \tilde{\tau} \\
& =\left\{\begin{aligned}
\left(\tau-\tau_{i n c}\right) \beta\left(\tau \mid \tau_{i n c}\right)+\frac{\alpha_{P} C \beta_{0}}{n}\left[\frac{k_{p} \tau_{i n c}}{k_{i n c}}\left(1-F_{\text {Beta }}\left(1-\tau / \tau_{i n c} ; k_{P}+1, k_{E}\right)\right)\right], & 0 \leq \tau<\tau_{\text {inc }}, \\
\left(\tau-\tau_{\text {inc }}\right) \beta\left(\tau \mid \tau_{\text {inc }}\right)+\frac{C \beta_{0}}{n}\left[\frac{\alpha k_{p} \tau_{i n c}}{k_{\text {inc }}}+\frac{1}{\mu} F_{\text {Gamma }}\left(\tau-\tau_{\text {inc }} ; k_{I}+1, \frac{1}{k_{I} \mu}\right)\right], & \tau \geq \tau_{\text {inc }},
\end{aligned}\right.
\end{aligned}
$$

where $F_{\text {Gamma }}(x ; a, b)$ is the cumulative distribution of a gamma distributed random variable with shape parameter $a$ and scale parameter $b$. The total force of infection exerted on each household member (over the course of infection) is then 


$$
B\left(\infty \mid \tau_{i n c}\right)=\frac{\beta_{0}}{n}\left(\frac{\alpha_{P} k_{P} \gamma \mu \tau_{i n c}+k_{\text {inc }} \gamma}{\alpha_{P} k_{P} \mu+k_{\text {inc }} \gamma}\right)
$$

1259 The mean of this expression over the incubation period distribution is $\beta_{0} / n$.

1260

1261 For a host who remains asymptomatic throughout infection, conditional on the combined

1262 duration of the $E$ and $P$ stages, $\tau_{\text {inc }}=y_{E}+y_{P}$, the infectiousness, $\beta\left(\tau \mid \tau_{\text {inc }}\right)$, is given by $1263 \alpha_{A}$ times the corresponding expression for a host who develops symptoms. We note that in

1264 this case, $\tau_{\text {inc }}$ has no epidemiological interpretation, but this conditional infectiousness was 1265 useful when fitting the model to data (see "Parameter fitting" below).

1266

1267

\section{Generation time distribution}

1268 The generation time, $\tau_{g e n}$, can be written as

$$
\tau_{g e n}=y_{E}+y^{*},
$$

1269 where $y_{E}$ is the length of the latent $(E)$ stage, and $y^{*}$ is the time from the start of the

1270 presymptomatic infectious $(P)$ stage to the transmission occurring. As shown by (Hart et al., 1271 2021), $y^{*}$ has density

$$
f^{*}\left(y^{*}\right)=C\left(\alpha_{P}\left(1-F_{P}\left(y^{*}\right)\right)+\int_{0}^{y^{*}}\left(1-F_{I}\left(y^{*}-y_{P}\right)\right) f_{P}\left(y_{P}\right) \mathrm{d} y_{P}\right) .
$$

1272 Using this density, it can be shown that the moments of this distribution are

$$
E\left[\left(y^{*}\right)^{m}\right]=\frac{C}{m+1}\left(\alpha_{P} E\left[y_{P}^{m+1}\right]+E\left[\left(y_{P}+y_{I}\right)^{m+1}-y_{P}^{m+1}\right]\right)
$$

1273 In particular,

$$
E\left[y^{*}\right]=\frac{C}{2}\left(\alpha_{P} E\left[y_{P}^{2}\right]+2 E\left[y_{P}\right] E\left[y_{I}\right]+E\left[{y_{I}}^{2}\right]\right),
$$

1274 and

$$
\operatorname{Var}\left[y^{*}\right]=\frac{C}{3}\left(\alpha_{P} E\left[y_{P}^{3}\right]+3 E\left[y_{P}{ }^{2}\right] E\left[y_{I}\right]+3 E\left[y_{P}\right] E\left[{y_{I}}^{2}\right]+E\left[y_{I}^{3}\right]\right)-\left(E\left[y^{*}\right]\right)^{2} .
$$


1275 Note that for a gamma distributed random variable, $X \sim \operatorname{Gamma}(a, b)$, we have

$$
E\left[X^{m}\right]=\frac{\Gamma(a+m)}{\Gamma(a)} b^{m}=a(a+1) \ldots(a+(m-1)) b^{m}
$$

1276 Therefore, for gamma distributed stage durations, explicit expressions can be obtained for the 1277 mean and variance of the generation time distribution,

$$
\begin{aligned}
E\left[\tau_{\text {gen }}\right] & =E\left[y_{E}\right]+E\left[y^{*}\right], \\
\operatorname{Var}\left[\tau_{\text {gen }}\right] & =\operatorname{Var}\left[y_{E}\right]+\operatorname{Var}\left[y^{*}\right],
\end{aligned}
$$

1278 where the last equality holds because $y_{E}$ and $y^{*}$ are assumed to be independent.

1279

Proportion of presymptomatic transmissions

Among infectors who develop symptoms, the proportion of transmissions occurring prior to symptom onset is given by (Hart et al., 2021)

$$
q_{P}=\frac{\left(\frac{\beta_{P} k_{P}}{k_{\text {inc }} \gamma}\right)}{\left(\frac{\beta_{P} k_{P}}{k_{\text {inc }} \gamma}+\frac{\beta_{I}}{\mu}\right)}=\frac{\alpha_{P} k_{P} \mu}{\alpha_{P} k_{P} \mu+k_{\text {inc }} \gamma} .
$$

\section{Parameter fitting}

The vector of model parameters,

$$
\theta=\left(k_{E} / k_{\text {inc }}, 1 / \mu, \alpha_{P}, \beta_{0}\right),
$$

was estimated by fitting the mechanistic model to the household transmission data.

We assumed independent prior distributions for each entry of $\theta$. Lognormal priors were assumed for $1 / \mu, \alpha_{P}$ and $\beta_{0}$. Since $\alpha_{P}$ represents the ratio between the transmission rates of hosts in the $P$ and $I$ stages, a prior with median 1 was used to ensure equal prior probabilities of values above and below 1 . This prior was also chosen to limit the prior probability of extreme values, with a prior $95 \%$ credible interval of $[0.2,5]$. A beta prior was used for 
$k_{E} / k_{\text {inc }}$ (which was constrained to lie between 0 and 1), and was chosen to restrict the prior

1294

1295

1296

1297

1298

1299

1300

1301

1302

1303

1304

1305

1306

1307

1308

1309

1310

1311

1312

1313

1314

probability of values very close to either 0 or 1 . The exact priors we used are given in

Appendix 1-table 3.

A slightly amended version of the parameter fitting algorithm described in the main text for the independent transmission and symptoms model was used. In particular, we augmented the observed data with:

i. The infection time, $t_{j}$, of each infected host.

ii. The time, $t_{s, j}$, at which each infected host transitioned from the $P$ to $I$ stage.

Note that for hosts who develop symptoms, the time of entry into the $I$ stage corresponds to the symptom onset time. The data were also augmented with this transition time for entirely asymptomatic infected hosts because the conditional infectiousness, $\beta\left(\tau \mid t_{s, j}-t_{j}\right)$, is more straightforward to calculate than $\beta(\tau)$.

In each step of the chain, we carried out (in turn) one of the following:

1. Propose new values for each entry of the vector of model parameters, $\theta$, using a multivariate normal proposal distribution (around the value of $\theta$ in the previous step of the chain; a correlation of 0.5 was used between the proposal distributions of $k_{E} / k_{\text {inc }}$ and $\alpha_{P}$, and between those of $1 / \mu$ and $\alpha_{P}$ ). Accept the proposed parameters, $\theta_{\text {prop }}$, with probability

$$
\min \left(\frac{L\left(\theta_{\text {prop }} ; \boldsymbol{t}\right) \pi\left(\theta_{\text {prop }}\right)}{L\left(\theta_{\text {old }} ; \boldsymbol{t}\right) \pi\left(\theta_{\text {old }}\right)}, 1\right)
$$

where $\theta_{\text {old }}$ denotes the vector of parameter values from the previous step of the chain, and where the augmented data, $t$, remain unchanged in this step. 
2. Propose new values for the precise symptom onset times of each symptomatic infected host, using independent uniform proposal distributions (within the day of symptom of onset for each host). For each household, $m$, accept the proposed augmented data, $\boldsymbol{t}_{\text {prop }}^{(m)}$, from that household with probability

$$
\min \left(\frac{L^{(m)}\left(\theta ; \boldsymbol{t}_{\text {prop }}^{(m)}\right)}{L^{(m)}\left(\theta ; \boldsymbol{t}_{\text {old }}^{(m)}\right)}, 1\right)
$$

where $\boldsymbol{t}_{\text {old }}^{(m)}$ denotes the corresponding augmented data from the previous step of the chain, and where the model parameters, $\theta$, remain unchanged in this step (i.e., proposed times are accepted/rejected independently for each household, according to the likelihood contribution from that household).

3. Propose new values for the infection time of one randomly chosen infected host in each household (either symptomatic or asymptomatic), using independent normal proposal distributions (around the equivalent times in the previous step of the chain). For each household, $m$, accept the proposed augmented data, $\boldsymbol{t}_{\text {prop }}^{(m)}$, from that household with probability

$$
\min \left(\frac{L^{(m)}\left(\theta ; \boldsymbol{t}_{\text {prop }}^{(m)}\right)}{L^{(m)}\left(\theta ; \boldsymbol{t}_{\text {old }}^{(m)}\right)}, 1\right)
$$

4. Propose new values for both the infection time, $t$, and the time of the start of the $I$ stage, $t_{s}$, holding $t_{s}-t$ constant, for one randomly chosen asymptomatic infected host in each household (in households where there was at least one), using independent normal proposal distributions (around the equivalent times in the previous step of the chain). For each household, $m$, accept the proposed augmented data, $\boldsymbol{t}_{\text {prop }}^{(m)}$, from that household with probability 


$$
\min \left(\frac{L^{(m)}\left(\theta ; \boldsymbol{t}_{\text {prop }}^{(m)}\right)}{L^{(m)}\left(\theta ; \boldsymbol{t}_{\text {old }}^{(m)}\right)}, 1\right)
$$

1335 Relationship between generation time, TOST and serial interval

1336 Here, we consider a randomly chosen infector-infectee pair (in which both the infector and

1337 the infectee develop symptoms) within a large, well-mixed population, of which only a small

1338 proportion is infected. In that setting, the observed generation time distribution is equal to the 1339 normalised infectiousness profile, which will not be true within a household (cf. Figure 1 and 1340 Figure 1-figure supplement 4). We define:

$$
\begin{aligned}
\tau_{\text {inc }, 1} & =\text { (incubation period of the infector) }, \\
\tau_{\text {inc }, 2} & =\text { (incubation period of the infectee) }, \\
\tau_{\text {gen }} & =\text { (generation time) }, \\
x_{\text {tost }} & =(\text { time from onset of symptoms (of infector) to transmission (TOST) }), \\
x_{\text {ser }} & =\text { (serial interval) },
\end{aligned}
$$

1341 where we use $\tau$ for time intervals relative to the time of infection and $x$ for those relative to

1342 the time of symptom onset. We denote the probability density functions of these time periods

1343 by $f_{\text {inc }, 1}, f_{\text {inc }, 2}, f_{\text {gen }}, f_{\text {tost }}$ and $f_{\text {ser }}$, respectively. Note that

$$
x_{\text {tost }}=\tau_{\text {gen }}-\tau_{\text {inc }, 1},
$$

1344 and

$$
x_{\text {ser }}=x_{\text {tost }}+\tau_{\text {inc }, 2} \text {, }
$$

1345 so that

$$
x_{\text {ser }}=\tau_{\text {gen }}+\tau_{\text {inc, } 2}-\tau_{\text {inc, } 1} .
$$


In the independent transmission and symptoms model, $\tau_{g e n}$ and $\tau_{\text {inc }, 1}$ are assumed to be

1348 independent, and the incubation periods of the infector and infectee are assumed to be drawn

1349 independently from the population incubation period distribution, $f_{\text {inc }}=f_{\text {inc }, 1}=f_{\text {inc }, 2}$.

1350 Therefore, the TOST distribution is given by the convolution

$$
f_{\text {tost }}\left(x_{\text {tost }}\right)=\int_{0}^{\infty} f_{\text {gen }}\left(x_{\text {tost }}+\tau\right) f_{\text {inc }}(\tau) \mathrm{d} \tau . \#(1)
$$

1351 Assuming that $x_{\text {tost }}$ and $\tau_{i n c, 2}$ are independent, the serial interval distribution can be 1352 calculated from the TOST distribution as

$$
f_{\text {ser }}\left(x_{\text {ser }}\right)=\int_{0}^{\infty} f_{\text {tost }}\left(x_{\text {ser }}-\tau\right) f_{\text {inc }}(\tau) \mathrm{d} \tau . \#(2)
$$

1353 Note that

$$
E\left[x_{\text {ser }}\right]=E\left[\tau_{\text {gen }}\right]+E\left[\tau_{\text {inc }, 2}\right]-E\left[\tau_{\text {inc }, 1}\right]=E\left[\tau_{\text {gen }}\right]
$$

1354 i.e., the generation time and serial interval distributions have the same mean.

1355

1356 For the mechanistic model, we still have $f_{i n c, 2}=f_{\text {inc }}$, and the serial interval distribution can

1357 be calculated from the TOST distribution using equation (2). On the other hand, $\tau_{g e n}$ and

$1358 \tau_{i n c, 1}$ are not independent, so equation (1) connecting the TOST and generation time

1359 distributions for the independent transmission and symptoms model does not hold for the

1360 mechanistic model. As shown by (Hart et al., 2021), the TOST distribution for the

1361 mechanistic model is, instead, given by

$$
f_{\text {tost }}\left(x_{\text {tost }}\right)=\left\{\begin{array}{cc}
\alpha_{P} C\left(1-F_{P}\left(-x_{\text {tost }}\right)\right), & x_{\text {tost }}<0, \\
C\left(1-F_{I}\left(x_{\text {tost }}\right)\right), & x_{\text {tost }} \geq 0 .
\end{array}\right.
$$

1363 Further, under the mechanistic model, the expected number of presymptomatic transmissions 1364 generated by an infected host is dependent on their incubation period. As a result, the 
infector's incubation period does not follow the same distribution as that of the infectee. In particular, by Bayes' theorem, we have

$$
f_{i n c, 1}\left(\tau_{i n c, 1}\right)=p\left(\tau_{i n c, 1} \mid 1 \rightarrow 2\right)=\frac{p\left(1 \rightarrow 2 \mid \tau_{i n c, 1}\right) f_{i n c}\left(\tau_{i n c, 1}\right)}{p(1 \rightarrow 2)},
$$

1367 where we write $1 \rightarrow 2$ to denote the occurrence of the transmission from the infector to the

1368 infectee. Because we are here considering a large population, the probability of the

1369 transmission occurring is proportional to the overall infectiousness of the infector (integrated

1370 over the course of infection), $B(\infty)$, so we have

$$
f_{i n c, 1}\left(\tau_{i n c, 1}\right)=\frac{B\left(\infty \mid \tau_{i n c}\right) f_{i n c}\left(\tau_{i n c, 1}\right)}{B(\infty)}=\left(\frac{\alpha_{P} k_{P} \gamma \mu \tau_{i n c, 1}+k_{i n c} \gamma}{\alpha_{P} k_{P} \mu+k_{i n c} \gamma}\right) f_{i n c}\left(\tau_{i n c, 1}\right)
$$

1371

The expected incubation period of the infector is then

$$
E\left[\tau_{i n c, 1}\right]=\frac{1}{\gamma}+\frac{\alpha_{P} k_{P} \mu}{k_{i n c} \gamma\left(\alpha_{P} k_{P} \mu+k_{i n c} \gamma\right)}=E\left[\tau_{i n c, 2}\right]+\frac{q_{P}}{k_{\text {inc }} \gamma}
$$

1372

where $q_{P}$ is the proportion of transmissions occurring prior to symptom onset.

1373

1374 As a result of the above, the expected values of the generation time and serial interval in the 1375 mechanistic model are not equal. Instead, we have

$$
E\left[x_{\text {ser }}\right]=E\left[\tau_{\text {gen }}\right]-\frac{q_{P}}{k_{\text {inc }} \gamma}
$$

1376 Under the values of $k_{i n c}$ and $\gamma$ that we assumed (Appendix 1-table 1), this gives a mean 1377 generation time that is approximately $\left(1.6 \times q_{P}\right)$ days longer than the mean serial interval.

\section{Extension of framework to account for co-primary cases}

In most of our analyses, we assumed that each household transmission chain was infected from within the household. However, we also relaxed this assumption by extending our framework to account for the possibility of co-primary cases (Figure 1- 
figure supplement 5 and Figure 3-figure supplement 6). Rather than assuming that all

1385

1386

1387

1388

1389

1390

1391

1392 As in Materials and Methods, we here consider a household (of size $n$ ) in which $n_{I}$ household members become infected (of whom $n_{S}$ develop symptoms and $n_{A}$ remain

1394

1395

1396

1397

1398

1399

1400

1401

1402 each susceptible household member at time $t$ is

$$
\lambda(t)=\lambda_{p}(t)+\lambda_{h}(t)
$$

1403 where

$$
\lambda_{p}(t)=\left\{\begin{aligned}
\beta_{p}, & t_{p, \text { start }} \leq t \leq t_{p, \text { end }} \\
0, & \text { otherwise }
\end{aligned}\right.
$$

1404 The cumulative force of infection is

$$
\Lambda(t)=\Lambda_{p}(t)+\Lambda_{h}(t)
$$


where

$$
\Lambda_{p}(t)=\int_{-\infty}^{t} \lambda_{p}(s) \mathrm{d} s=\frac{\beta_{p}}{2}\left(t_{p, \text { end }}-t_{p, \text { start }}+\left|t-t_{p, \text { start }}\right|-\left|t_{p, \text { end }}-t\right|\right) .
$$

1406 We took $t_{p, s t a r t}$ and $t_{p, e n d}$ to be the start and end of the day of the first household

1407 member becoming infected, respectively.

1408

1409 The likelihood contribution from the household, $L(\theta)$, where $\theta$ is the vector of

1410 unknown model parameters, is then given by

$$
L(\theta)=\frac{1}{1-\exp \left(-n \beta_{p}\left(t_{p, \text { end }}-t_{p, \text { start }}\right)\right)} \prod_{k=1}^{n} L_{k, 1}(\theta) L_{k, 2}(\theta) .
$$

1411 Here,

$$
L_{k, 1}(\theta)=\left\{\begin{aligned}
\lambda\left(t_{k}\right) \exp \left(-\Lambda\left(t_{k}\right)\right), & \text { for } k=1, \ldots, n_{I} \\
\exp (-\Lambda(\infty)), & \text { for } k=n_{I}+1, \ldots, n
\end{aligned}\right.
$$

1412 and for the independent transmission and symptoms model,

$$
L_{k, 2}(\theta)=\left\{\begin{aligned}
f_{\text {inc }}\left(t_{s, k}-t_{k}\right), & \text { if host } k \text { becomes infected and develops symptoms; } \\
1, & \text { otherwise; }
\end{aligned}\right.
$$

1413 where $f_{\text {inc }}$ is the probability density function of the incubation period, while for the

1414 mechanistic model,

$$
L_{k, 2}(\theta)=\left\{\begin{aligned}
f_{\text {inc }}\left(t_{s, k}-t_{k}\right), & \text { for } k=1, \ldots, n_{I} \\
1, & \text { for } k=n_{I}+1, \ldots, n
\end{aligned}\right.
$$

1415 The factor

$$
\frac{1}{1-\exp \left(-n \beta_{p}\left(t_{p, \text { end }}-t_{p, \text { start }}\right)\right)}
$$

1416 is included to condition on at least one household member becoming infected during

1417 the primary transmission event. 
1419 Using this likelihood function, we fitted both models to the household data using the 1420 same data augmentation MCMC approach described for the independent transmission 1421 and symptoms model in Materials and Methods and for the mechanistic model earlier

1422 in the Appendix. Alongside other model parameters, we estimated the probability of

1423 each household member becoming infected during the primary transmission event,

$$
1-\exp \left(-\beta_{p}\left(t_{p, \text { end }}-t_{p, \text { start }}\right)\right)
$$

1424 in the MCMC procedure (in the case we considered, $\left(t_{p, \text { end }}-t_{p, \text { start }}\right)$ was always equal to

1425 one day, so $\beta_{p}$ could be calculated from this probability). A uniform prior was assumed for 1426 the probability of primary infection. 


\begin{tabular}{|c|c|c|c|c|}
\hline Parameter & Model & Interpretation & Value & Justification \\
\hline$\alpha_{A}$ & Both & $\begin{array}{c}\text { Relative } \\
\text { infectiousness } \\
\text { of entirely } \\
\text { asymptomatic } \\
\text { hosts }\end{array}$ & 0.35 & $\begin{array}{c}\text { Taken from } \\
\text { (Buitrago- } \\
\text { Garcia et al., } \\
\text { 2020) (other } \\
\text { values } \\
\text { considered in } \\
\text { sensitivity } \\
\text { analyses) }\end{array}$ \\
\hline $\begin{array}{c}\text { Mean of natural } \\
\text { logarithm of the } \\
\text { incubation } \\
\text { period }\end{array}$ & $\begin{array}{c}\text { Independent } \\
\text { transmission } \\
\text { and symptoms }\end{array}$ & $\begin{array}{l}\text { Parameter of } \\
\text { lognormal } \\
\text { incubation } \\
\text { period } \\
\text { distribution }\end{array}$ & $1.63 \log$ (day) & $\begin{array}{l}\text { Taken from } \\
\text { (McAloon et } \\
\text { al., 2020) } \\
\text { (uncertainty in } \\
\text { this value } \\
\text { considered in } \\
\text { sensitivity } \\
\text { analyses) }\end{array}$ \\
\hline $\begin{array}{c}\text { Standard } \\
\text { deviation of } \\
\text { natural } \\
\text { logarithm of the } \\
\text { incubation } \\
\text { period }\end{array}$ & $\begin{array}{c}\text { Independent } \\
\text { transmission } \\
\text { and symptoms }\end{array}$ & $\begin{array}{l}\text { Parameter of } \\
\text { lognormal } \\
\text { incubation } \\
\text { period } \\
\text { distribution }\end{array}$ & $0.50 \log ($ day $)$ & $\begin{array}{l}\text { Taken from } \\
\text { (McAloon et } \\
\text { al., 2020) } \\
\text { (uncertainty in } \\
\text { this value } \\
\text { considered in } \\
\text { sensitivity } \\
\text { analyses) }\end{array}$ \\
\hline$k_{\text {inc }}$ & Mechanistic & $\begin{array}{c}\text { Shape } \\
\text { parameter of } \\
\text { gamma } \\
\text { incubation } \\
\text { period } \\
\text { distribution }\end{array}$ & 3.5 & $\begin{array}{l}\text { Consistent with } \\
\text { mean and } \\
\text { standard } \\
\text { deviation from } \\
\text { (McAloon et } \\
\text { al., 2020) }\end{array}$ \\
\hline $1 / \gamma$ & Mechanistic & $\begin{array}{c}\text { Mean } \\
\text { incubation } \\
\text { period }\end{array}$ & 5.8 days & $\begin{array}{l}\text { Consistent with } \\
\text { mean and } \\
\text { standard } \\
\text { deviation from } \\
\text { (McAloon et } \\
\text { al., 2020) } \\
\end{array}$ \\
\hline$k_{I}$ & Mechanistic & $\begin{array}{c}\text { Shape } \\
\text { parameter of } \\
\text { (gamma) } \\
\text { symptomatic } \\
\text { infectious } \\
\text { period } \\
\text { distribution }\end{array}$ & 1 & Assumed \\
\hline
\end{tabular}

1430 Appendix 1-table 1. Assumed (not fitted) parameter values used for the two models that we considered. 


\begin{tabular}{|c|c|c|}
\hline Parameter & Prior & Posterior mean (95\% CrI) \\
\hline $\begin{array}{c}\text { Mean generation } \\
\text { time }\end{array}$ & $\begin{array}{c}\text { Lognormal(1.6,0.35) } \\
\text { [prior median 5.0 days, 95\% } \\
\text { CrI 2.5-9.8 days] }\end{array}$ & $\begin{array}{c}4.2 \text { days } \\
(3.3-5.3 \text { days })\end{array}$ \\
\hline $\begin{array}{c}\text { Standard deviation } \\
\text { of generation time } \\
\text { distribution }\end{array}$ & $\begin{array}{c}\text { Lognormal(0.7,0.65) } \\
\text { [prior median 2.0 days, 95\% } \\
\text { CrI 0.6-7.2 days] }\end{array}$ & $\begin{array}{c}4.9 \text { days } \\
(3.0-8.3 \text { days })\end{array}$ \\
\hline $\begin{array}{c}\text { Overall } \\
\text { infectiousness } \\
\text { parameter, } \beta_{0}\end{array}$ & $\begin{array}{c}\text { Lognormal(0.7,0.8) } \\
\text { [prior median 2.0, 95\% CrI } \\
0.4-9.7]\end{array}$ & 1.7 \\
\hline
\end{tabular}

1432 Appendix 1-table 2. Fitted parameters in the independent transmission and symptoms model, the prior 1433 distributions used, and the posterior means and 95\% credible intervals obtained. 


\begin{tabular}{|c|c|c|}
\hline Parameter & Prior & Posterior mean (95\% CrI) \\
\hline $\begin{array}{l}\text { Ratio of mean durations of } \\
\text { the latent }(E) \text { and incubation } \\
(E+P) \text { periods, } k_{E} / k_{\text {inc }}\end{array}$ & $\begin{array}{c}\operatorname{Beta}(2.1,2.1) \\
\text { [prior median 0.5, 95\% CrI } \\
0.1-0.9]\end{array}$ & $\begin{array}{c}0.2 \\
(0.03-0.5)\end{array}$ \\
\hline $\begin{array}{c}\text { Mean symptomatic } \\
\text { infectious }(I) \text { period, } 1 / \mu\end{array}$ & $\begin{array}{l}\text { Lognormal }(1.6,0.8) \\
\text { [prior median } 5.0 \text { days, } 95 \% \\
\text { CrI 1.0-23.8 days] }\end{array}$ & $\begin{array}{c}5.0 \text { days } \\
(3.2-7.5 \text { days })\end{array}$ \\
\hline $\begin{array}{l}\text { Ratio of transmission rates } \\
\text { in the } P \text { and } I \text { stages, } \alpha_{P}\end{array}$ & $\begin{array}{c}\text { Lognormal }(0,0.8) \\
\text { [prior median } 1.0,95 \% \mathrm{CrI} \\
0.2-4.8]\end{array}$ & $\begin{array}{c}3.1 \\
(1.2-6.9)\end{array}$ \\
\hline $\begin{array}{l}\text { Overall infectiousness } \\
\text { parameter, } \beta_{0}\end{array}$ & $\begin{array}{c}\text { Lognormal }(0.7,0.8) \\
\text { [prior median } 2.0,95 \% \mathrm{CrI} \\
0.4-9.7]\end{array}$ & $\begin{array}{c}1.8 \\
(1.5-2.1)\end{array}$ \\
\hline
\end{tabular}

1436 Appendix 1-table 3. Fitted parameters in the mechanistic model, the prior distributions used, and the posterior 1437 means and 95\% credible intervals obtained. 


\begin{tabular}{|c|c|c|c|}
\hline Model & Distribution & Mean & $\begin{array}{c}\text { Standard } \\
\text { deviation }\end{array}$ \\
\hline \multirow{2}{*}{$\begin{array}{c}\text { Independent } \\
\text { transmission } \begin{array}{c}\text { sand } \\
\text { symptoms }\end{array}\end{array}$} & Generation time & 4.2 days & 4.9 days \\
\cline { 2 - 4 } & TOST & -1.6 days & 5.8 days \\
\hline \multirow{2}{*}{ Mechanistic } & Serial interval & 4.2 days & 6.6 days \\
\cline { 2 - 4 } & Generation time & 5.9 days & 4.8 days \\
\cline { 2 - 4 } & TOST & -1.1 days & 4.9 days \\
\cline { 2 - 4 } & Serial interval & 4.7 days & 5.8 days \\
\hline
\end{tabular}

1440 Appendix 1-table 4. The means and standard deviations of the generation time, TOST and serial interval

1441 distributions shown in Figure 2. Other than the generation time distribution for the independent transmission and

1442 symptoms model (which is lognormal with the specified mean and standard deviation), none of the remaining

1443 distributions take a simple parametric form. 